\title{
Nondestructive Condition Assessment of Civil Infrastructure Using Ultrasonic Technique
}

Tyler Keith Ashbrook

tka0003@mix.wvu.edu

Follow this and additional works at: https://researchrepository.wvu.edu/etd

Part of the Civil Engineering Commons, and the Structural Engineering Commons

\section{Recommended Citation}

Ashbrook, Tyler Keith, "Nondestructive Condition Assessment of Civil Infrastructure Using Ultrasonic Technique" (2021). Graduate Theses, Dissertations, and Problem Reports. 10340.

https://researchrepository.wvu.edu/etd/10340

This Problem/Project Report is protected by copyright and/or related rights. It has been brought to you by the The Research Repository @WVU with permission from the rights-holder(s). You are free to use this Problem/Project Report in any way that is permitted by the copyright and related rights legislation that applies to your use. For other uses you must obtain permission from the rights-holder(s) directly, unless additional rights are indicated by a Creative Commons license in the record and/ or on the work itself. This Problem/Project Report has been accepted for inclusion in WVU Graduate Theses, Dissertations, and Problem Reports collection by an authorized administrator of The Research Repository @ WVU. For more information, please contact researchrepository@mail.wvu.edu. 


\title{
Nondestructive Condition Assessment of Civil Infrastructure Using Ultrasonic Technique
}

\author{
Tyler Ashbrook
}

\author{
Problem Report submitted to the \\ Benjamin M. Statler College of Engineering and Mineral Resources \\ at West Virginia University \\ in partial fulfillment of the requirements \\ for the degree of
}

Master of Science
in
Civil Engineering

Udaya B. Halabe, Ph.D., P.E., Chair

Hema J. Siriwardane, Ph.D., P.E.

Bhaskaran Gopalakrishnan, Ph.D., P.E.

Wadsworth Department of Civil and Environmental Engineering

Morgantown, West Virginia

2021

Keywords: Nondestructive Testing; NDT; Ultrasonic; Pulse Echo; Pulse Velocity; Phased Array; Concrete; Steel; Weld; Timber

Copyright 2021 Tyler Ashbrook 


\title{
ABSTRACT \\ Nondestructive Condition Assessment of Civil Infrastructure Using Ultrasonic Technique
}

\author{
Tyler Ashbrook
}

Civil Infrastructure controls the way people live by providing safe passage and shelter across the entire globe. Since safety leads the concern in infrastructure, it is essential to ensure that these structures have been adequately tested and analyzed periodically. Testing these structures can be extremely expensive when parts are required to be removed to capture the flaw, which is why nondestructive testing (NDT) continues to grow in demand since it can evaluate the structural component in-situ without causing any damage. One of the leading methods of nondestructive condition assessment uses ultrasonic technique to capture flaws in the system without damaging the structure. Ultrasonic testing (UT) helps to adequately sustain and advance the safety in existing and future infrastructure across the globe. Continual advancement and use of UT methods for the application of condition assessment in the field of civil infrastructure can provide a cheaper alternative to other conventional practices.

This report focuses on the UT methods of NDT and aims to provide an in-depth literature review of the different testing styles. Ultrasonic Pulse Velocity, Ultrasonic Pulse Echo, Semi-direct and Indirect testing, Time of flight Diffraction, Phased Array ultrasonic testing, are some of the NDT methods commonly used and discussed in the report. These techniques are routinely being applied to the condition assessment of concrete, steel, and timber structural members including the assessment of debonding between rebar cage and surrounding concrete in reinforced concrete structural members. With the techniques already established, the goals of the NDT industry are to make the techniques safer, cost effective, and modifying the systems to integrate a more accessible process. As the future of NDT rapidly expands, it is imperative that the systems are manageable and effective when a non-specialist entity operates them in the field. Managing the extensive knowledge and application of these techniques can lead to enhanced material and construction quality. 


\section{ACKNOWLEDGEMENTS}

I would like to acknowledge my academic and research advisor, Dr. Udaya B. Halabe, for his consistent support and assistance during my Master of Science in Civil Engineering (MSCE) degree program. I am extremely honored to have Dr. Halabe not only guide me with helpful insight towards completely my degree, but also using his time to teach valuable material about civil infrastructure and nondestructive testing methods.

I would like to thank Dr. Hema J. Siriwardane and Dr. Bhaskaran Gopalakrishnan, for serving as members of my Advisory and Examining Committee and for providing insightful suggestions.

I would like to sincerely thank Ms. Cate Schlobohm for providing a welcoming and educational office. I am extremely grateful Ms. Schlobohm took the time and patience to help develop me as a person and worker. The experience I received learning from her cannot be achieved anywhere else.

I also wish to acknowledge the Wadsworth Department of Civil and Environmental Engineering and the Statler College at West Virginia University for the educational experience, resources and financial support that I received in the form of assistantships, opportunities, and tuition waivers during my MSCE degree program.

Finally, I would like to take this opportunity to thank my family (Mr. Brian Ashbrook, Ms. Debbie Ashbrook) for providing me the opportunity to achieve a college degree at the BS and MS level with their continual support. 


\section{TABLE OF CONTENTS}

ABSTRACT ..................................................................................................................................................... ii

ACKNOWLEDGEMENTS ................................................................................................... iii

LIST OF FIGURES .....................................................................................................

LIST OF TABLES ........................................................................................................................ ix

1. INTRODUCTION.......................................................................................................................................1

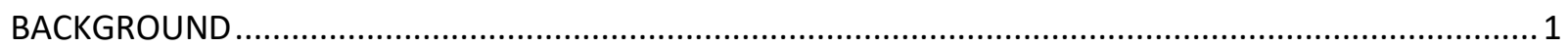

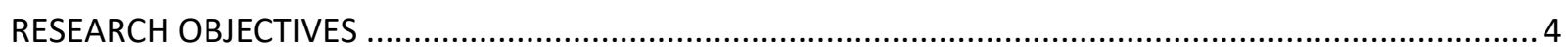

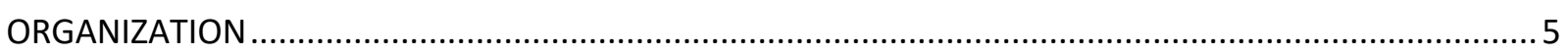

2. ULTRASONIC TESTING OVERVIEW …......................................................................6

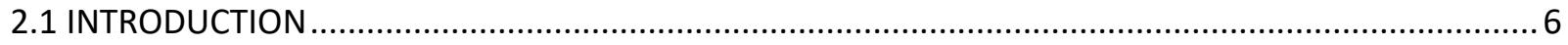

2.2 EQUIPMENT COMMONLY USED IN ULTRASONIC TESTING METHODS ...................................... 10

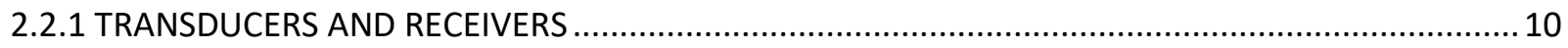

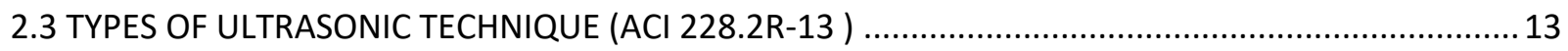

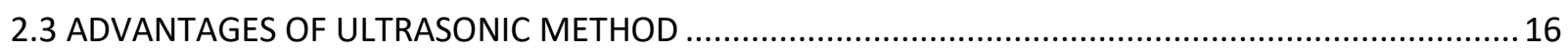

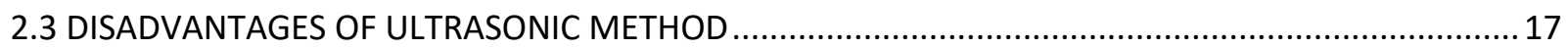

3. CONDITION ASSESSMENT IN CONCRETE MATERIALS .............................................19

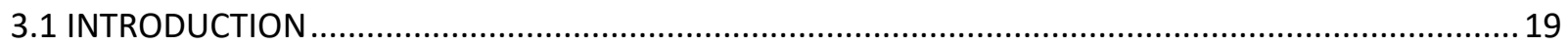

3.2 REVIEW OF APPLICATIONS OF ULTRASONIC TECHNIQUES IN DIFFERENT CONCRETE MATERIALS.. 23

3.2.1 PREDICTION OF REINFORCED CONCRETE STRENGTH BY ULTRASONIC VELOCITIES (SABBAG \&

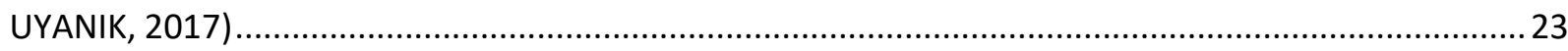

3.2.2 ULTRASONIC TESTING OF REACTIVE POWDER CONCRETE (WASHER ET AL. 2004) ......................27

3.2.3 FAST AND REAL TIME INSPECTION OF CONCRETE STRUCTURES USING A ROLLING 3D SCANNER

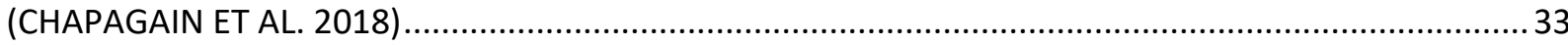

3.2.4 ULTRASONIC ASSESSMENT OF THE CONCRETE RESIDUAL STRENGTH AFTER A REAL FIRE EXPOSURE (WROBLEWSKI, R., \& STAWISKI, B., 2020) .................................................................... 35

3.2.5 CONDITION ASSESSMENT OF CONCRETE ELEMENTS THROUGH TWO NONDESTRUCTIVE

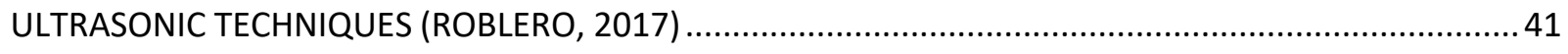

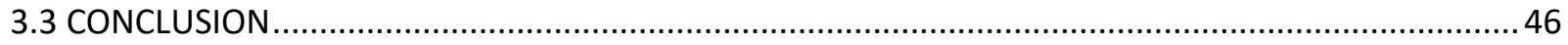

4. APPLICATIONS OF ULTRASONIC TECHNIQUES ON STEEL MATERIALS .........48

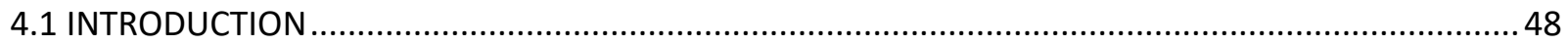

4.2 REVIEW OF APPLICATIONS OF ULTRASONIC TECHNIQUES IN DIFFERENT STEEL MATERIALS...........51

4.2.1 A BAYESIAN ESTIMATION-BASED UNCERTAINTY QUANTIFICATION OF FLAWS IN STEEL WELDS DETECTED BY ULTRASOUND PHASED ARRAY (HE ET AL. 2020) ........................................................ 51 
4.2.3 INTERPRETATION OF INDICTATIONS BY SMALL WELDING DISCONTINUITIES IN ULTRASONIC TIME OF FLIGHT DIFFRACTION TECHNIQUE (KACZMAREK ET AL. 2018) .56

4.2.4 A POSSIBILITY OF USING TRANSVERSE ULTRASONIC WAVES TO OBTAIN INFORMATION ON

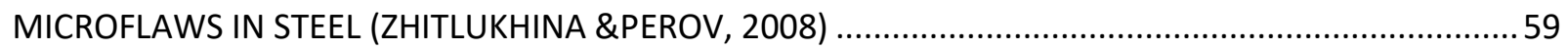

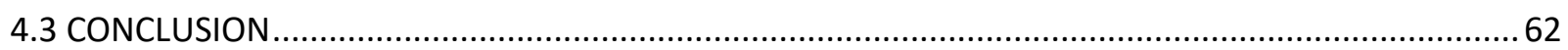

\section{CONDITION ASSESSMENT IN TIMBER STRUCTURES ........................................64}

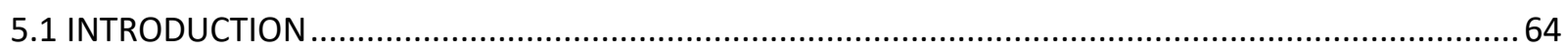

5.2 REVIEW OF APPLICATIONS OF ULTRASONIC TECHNIQUES IN DIFFERENT TIMBER STRUCTURES ...66

5.2.1 ASSESSMENT OF THE CONDITION OF WHARF TIMBER SHEET WALL MATERIAL BY MEANS OF SELECTED NON-DESTRUCTIVE METHODS (NOWAK ET AL. 2019) .....................................................66

5.2.2 SPACING FOR ACCURACY IN ULTRASONIC TESTING OF BRIDGE TIMBER PILES (MCUEN ET AL.

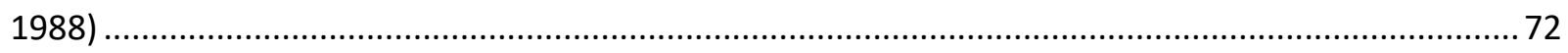

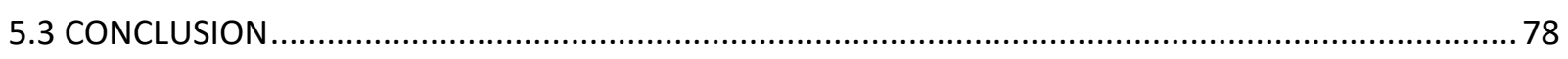

6. CONCLUSIONS AND RECOMMENDATIONS....................................................79

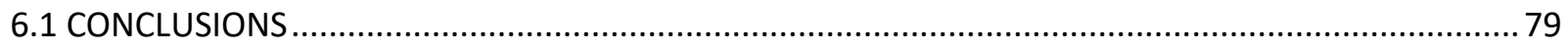

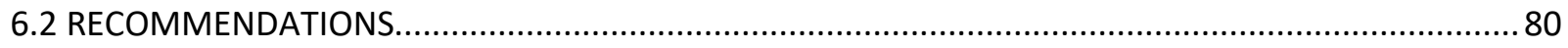

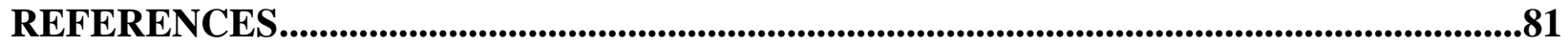




\section{LIST OF FIGURES}

Figure 2.1: Ultrasonic transducer applications in other fields. (Stephens, 1975) ...................... 7

Figure 2.2: Longitudinal waves showing excited zones. (Krautkrämer et al, 2013) ................... 8

Figure 2.3: Transverse or Shear (S-waves) from left to right (Krautkrämer et al, 2013) ............. 9

Figure 2.4: Cut away of standard transducer used in ultrasonic testing (Kishore, 2014) ............ 10

Figure 2.5: Angle Beam Transducer Position (Kishore, 2014) ............................................ 12

Figure 2.6: Ultrasonic Velocity test example and transducer setup (Zielinska \& Rucka, 2020).. 14

Figure 2.7:Example of ultrasonic pulse echo method setup (Tian et al. 2019) ......................... 15

Figure 3.1: Example of spalling and cracking in concrete samples (Roblero, 2017) ................ 21

Figure 3.2: Demonstration of sample cubes being evaluated for testing (Sabbag \& Uyanik, 2017)

Figure 3.3: Comparison charts for P-wave velocity, S-wave velocity, and Uniaxial Compressive

Strength with different reinforcing diameters (Sabbag \& Uyanik, 2017) ................................ 26

Figure 3.4:Multilayered Ultrasonic Rolling Scanner built by ELOP (Chapagain et al. 2018) ..... 33

Figure 3.5: Example of $20 \mathrm{~mm}$ slab with a defect at a depth of $15 \mathrm{~cm}$ (Chapagain et al. 2018).. 34

Figure 3.6: Concrete block with a steel rebar placed at $12.5 \mathrm{~cm}$ depth (Chapagain et al. 2018).. 35

Figure 3.7: (a) Steel rebar exposure (b) color change after effects of fire exposure on core sample

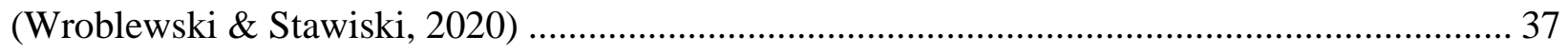

Figure 3.8: Face of 64-apartment complex building after exposure to fire (Wroblewski \& Stawiski,

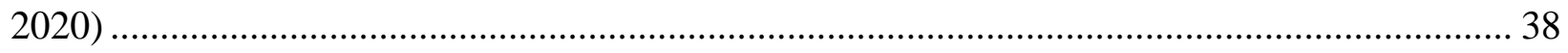

Figure 3.9: Example setup of Unipan 543 digital device and placement of ultrasonic point heads

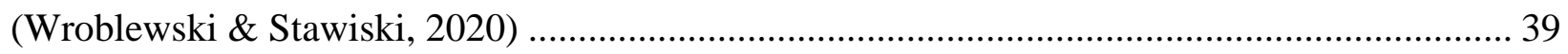

Figure 3.10: Strength of concrete sample after exposure to fire based on depth of testing

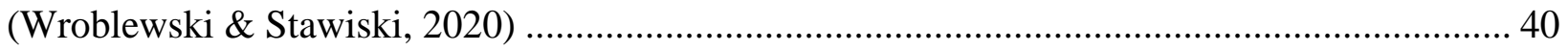

Figure 3.11: Computed velocities for grease only coupling (Roblero, 2017) ......................... 44 
Figure 3.12: Computed velocities for a grease and duct tape coupling system (Roblero, 2017) . 44

Figure 3.13: Velocities for a sound concrete sample (Roblero, 2017) ................................. 45

Figure 3.14: Velocities for a concrete sample with known defects (Roblero, 2017)................. 46

Figure 4.1: Two separate tests conducted on the same weld (He et al. 2020 ) ......................... 52

Figure 4.2: Averaged result of the given steel weld (He et al. 2020) ....................................... 52

Figure 4.3: Flaws for steel welds at $95 \%$ and $99 \%$ confidence intervals (He et al. 2020) .......... 53

Figure 4.4: Setup for ultrasonic testing method showing the three different locations of testing $(\mathrm{Gu}$

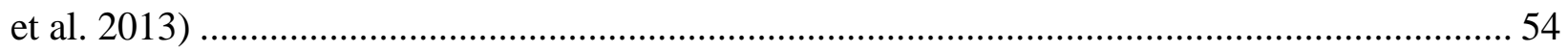

Figure 4.5: Comparison between real measured weld widths and calculate data at $30 \%$ attenuation

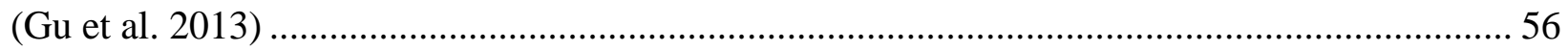

Figure 4.6: Setup for TOFD and equipment used in experiment (Kaczmarek et al. 2018) ......... 57

Figure 4.7: OmniPC software displaying the determination and depth of discontinuities

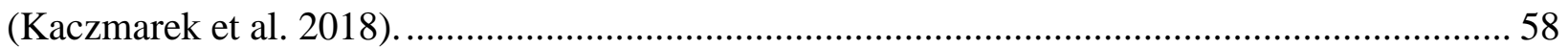

Figure 4.8: Experimental and calculated data for the three specimen with different defect sizes and

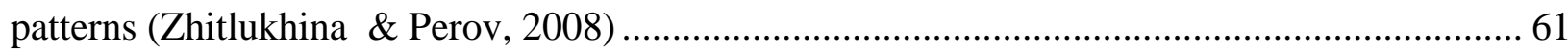

Figure 4.9: Elastic displacements in a trasnverse wave for the (a) inside of the specimen and (b)

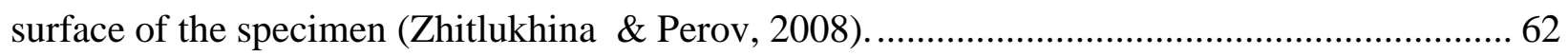

Figure 5.1: Detailed view of piles dismantled and the different zones where the pile was affected (Nowak et al. 2019). 67

Figure 5.2: Example setup for (a) the Fakopp Microsecond Timer and (b) Sylvatest Trio Device (Nowak et al. 2019). 69

Figure 5.3: Shown are (a) sample taken for test. (b) Sample for tests with a cut (Nowak et al. 2019) 70

Figure 5.4: Cross-section of timber bridge pile showing setup for the ultrasonic testing (McCuen et al. 1988) 74 
Figure 5.5: Relative error of estimation between sampling points separated by distance (McCuen,

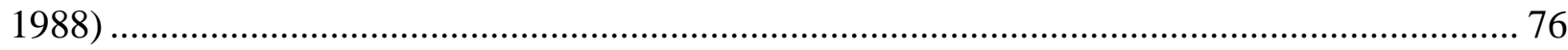

Figure 5.6: Intervals and Kriging estimations (McCuen et al, 1988) .................................... 77 


\section{LIST OF TABLES}

Table 3.1: Common deterioration methods with supporting information (Breysse, 2010)......... 21

Table 3.2: Reactive Powder Concrete Mixture Sample (Washer et al. 2004 ) .......................... 27

Table 3.3: RPC Cube lengths and percentage of steel fibers in mixture (Washer et al. 2004).... 29

Table 3.4: Parameters for cylinders tested with steel fiber percentage (Washer et al. 2004) ....... 30

Table 3.5: Longitudinal and Shear waves tested at $500 \mathrm{kHz}$ (Washer et al. 2004) ................... 31

Table 3.6: Longitudinal and Shear waves tested at $1 \mathrm{MHz}$ (Washer et al. 2004)...................... 31

Table 3.7: Cylinder velocities in the $\mathrm{P}$ and $\mathrm{S}$-waves with and without steel fibers (Washer et al.

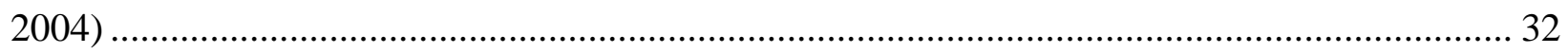

Table 4.1.8: Causes and remedies for porosity in welds (Genculu, 2007) .............................. 49

Table 4.2.9: Causes and Remedies for cracks in welds (Genculu, 2007) ................................ 50

Table 4.3.10: Summary table for TOFD and Metallographic Examination for different discontinuities in MAG weld (Kaczmarek et al. 2018) .......................................................... 59

Table 5.1.11: Different types of degradations for timber members (Nowak et al. 2019)............ 64

Table 5.2.12: Fakopp Microsecond Timer Results for the velocities and dynamic modulus of elasticity at different moisture contents (Nowak et al. 2019) ................................................ 71

Table 5.3.13: Sylvatest Trio results for the velocities and dynamic modulus of elasticity at different

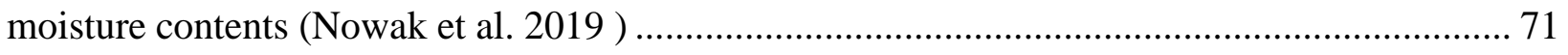

Table 5.4.14: Bridge piles with direction analysis was completed (McCuen, 1988) ................. 75 


\section{INTRODUCTION}

\section{BACKGROUND}

Infrastructure expansion in the United States is not the critical concern compared to the rehabilitation and maintenance of the existing structures. With the abundance of structures already standing around the country, the focus shifted slightly to keep an eye on these existing obstacles. The gap in the investment towards infrastructure repair continues to grow even with these new technologies advancing to a better system. Over the last 10 years, the gap in the infrastructure bill has increased by nearly half a trillion dollars, and will continue to grow at an exponential rate if the repairment does not improve. According to the American Society of Civil Engineers (ASCE), the United States infrastructure has a grading of a $\mathrm{C}$-, while the infrastructure bill is calculated from a grading of B standing ( https://infrastructurereportcard.org/). With the continuation in the spread of the gap in the infrastructure bill, civil infrastructure will continue to be affected by the snowball effect towards the quality of life (Bennon et al. 2017). Every structure from airports, roads, buildings, etc. need a more efficient and cheaper alternative to decelerate the deterioration in service life.

Nondestructive testing (NDT) encompasses a new, more advanced method to assess structural components since it does need to destroy a member to analyze for any defects. The testing can be applied in a range of fields such as infrastructure, medical imaging, electrical systems, etc. NDT methods vary depending on the signal conversations produced from the unit to the intended target to inspect, evaluate and collect without permanently altering the subject. The more commonly recognizable method of NDT, visual inspection, allows the specimen to be inspected on broad spectrum. Surface defects that are easily visible to the eye are studied using the visual method. NDT practices allow the in-situ properties to be evaluated and processed, whereas other methods cannot provide this detail report in the members. Structures analyzed by these NDT techniques benefit in repair time since fatal flaws can be caught before advancing to a deeper phase

of damage. Rapid repairs before structures reach a fatal state lead to cheaper costs for the rehabilitation to ensure a longer life span. 
NDT contains several unique testing methods that differentiate typically based on the equipment being used and the penetrating material. Each piece of equipment emits a different type of wave based on the properties and the needs for the experiment. Understanding these waves plays a large role in which test needs to take place. Other than the visual testing and UT methods, the most common NDT methods are Radiography, Eddy Current, Acoustic Emission, Leak Testing, Liquid Penetrant, Magnetic Particle, etc. Similar to other subjects, each test displays strengths and weaknesses directed towards a test based on where the experiment is taking place and sample being studied. A test may be completed using one of the methods, but another method may create a data set in an expediated time or reduced cost.

One of the goals for NDT is to prevent damage and extra construction in infrastructure by conducting inspections and tests to ensure safety. The I-35 Bridge in Minneapolis, Minnesota ultimately collapsed because of a design failure, but the disaster could have possibly been avoided if NDT inspection had been implemented. The bridge was opened in 1967 and had several rehabilitations done prior to the collapse of the structure. The bridge failed because of several different reasons, but the main one considered to be a gusset plate on the structure being half the size of the design. Safety factors allowed the bridge to stand for nearly 40 years before failing in 2007 (Hao, 2010). NDT could have potentially saved the bridge from collapse by analyzing the different stresses in the structure. The earlier the detection of the high stresses in the gusset plate, the quicker and cheaper the rehabilitation could have taken place.

Civil infrastructure has many failure methods, but one of the more common modes of damage could be found in corrosion. Rebar residing in the concrete members corrodes once exposed to water seeping through cracks in the surface of the concrete. Many bridges with corrosion damage are located on the coasts since the weather plays a stronger role in these areas. Corrosion located on bridge piers disrupts the strength when seismic events take place (Yuan et al. 2017). Even though corrosion does not typically cause the bridge to ultimately collapse, the weakened members play a large role in the downfall. Having the ability to prevent corrosion would be one of the bigger achievements in today's time since so much damage takes place. Since corrosion happens over a period of time, earlier detection creates a cheaper rehabilitation. NDT allows the corrosion to be found at early stages of development and create lower rates of repair. 
This report discusses the importance of NDT, but also focuses in depth on the Ultrasonic Testing (UT) methods, and how they improve the quality of life. UT uses a range of sound too intense for the human ear to process. These pitches in UT have frequencies higher than $20 \mathrm{kHz}$, which is greater than the frequency that a human ear can detect. The UT test functions properly by applying Hooke's Law to the structure to ensure the resultant strain is proportional to the applied stress overall. Low amplitude waves are propagated through a material to locate defects or flaws within. This information depends on the time the wave remains in the object, and whether the wave bounces in different directions (as if an impediment lies in the path of the travelling wave). Another advantage for applying the UT method relates to the accuracy at which the test can detect internal flaws within the member.

This report covers nondestructive testing on different concretes, steel and timber. The application of steel and concrete together (i.e., steel rebar bonded within the concrete) can be found in both sections of the concrete and steel. Concrete makes up a vast majority of the civil infrastructure in the United States making it highly susceptible to being unseen if damaged. Ultrasonic methods allow inspectors to cover a greater amount of structures at a heavily deduced cost. Concrete is one of the most durable materials used and produced in infrastructure, yet extremely brittle in certain conditions including being exposed to environmental settings. This report focuses on the damaged concrete and how ultrasonic techniques are able detect any detrimental effects from corrosion, various voids, fire, chemicals, and many other limiting factors.

While steel infrastructure usually consists of massive structures, this report focuses more on the smaller details (steel welds, connections for joints). Similar to reinforced concrete, steel structures exposed to water have a high rate of corrosion. Since corrosion cannot be avoided, detecting it, or predicting a lifespan model, improves the quality of life in the structure. Methods such as phased array ultrasonic testing or an ultrasonic additive manufacturing method, are suitable for these smaller steel connections/welds and increase the workability. In today's infrastructure, many systems contain multiple elements. Reinforced concrete ensures the success of the structures by adding steel rebar into the system. Once the outer shell of concrete shows any element of cracking, this exposes the reinforcement to phenomena's such as freeze and thawing, fire, corrosion, and other detrimental effects. These hindering effects are able to be detected and 
mapped (depending on the equipment) using UT methods. Other information including the debond interface and subsurface cracks are closely monitored since these are common issues.

Timber structures contain some of the long-standing practices when it comes to civil infrastructure. The material lacks the strength and size of steel or concrete, but the organic material maintains durable profile if performed correctly. Since timber has the natural components in it, weather and time compromise the material in different scenarios. Ultrasonic techniques analyze timber structures for several components such as elasticity and strength. Approaching the structure based on the age or moisture content help to provide information regarding these different parameters. Timber structures are susceptible to other factors such as insects and decay, that would not typically be seen in a steel or concrete member.

With the development of the world comes the evolvement and advancement of NDT, and more specifically ultrasonic testing. This report highlights areas where methods and equipment are altered and advanced to increase the production rate and alleviate negative aspects of testing. Cheaper production costs ultimately reduce the overall cost spent on civil infrastructure and provide a simpler method that allows structures to stand for a greater lifespan.

\section{RESEARCH OBJECTIVES}

Ultrasonic methods in the nondestructive testing field have made severe advancements over the years to bring a more cost-effective and capable way of assessing the condition of structural elements. The research objectives for this report are as follows:

$>$ To organize a literature review that analyzes the importance of the on-going advancement in the ultrasonic testing methods including ultrasonic pulse velocity, ultrasonic pulse echo, semi-direct and indirect testing, time of flight diffraction, phased array ultrasonic testing.

$>$ To conduct a literature review focusing on the modification of UT test methods for future projects that could decrease the cost and increase the efficiency of the condition assessment of civil infrastructure using NDT. 


\section{ORGANIZATION}

This problem report is organized into six chapters. Chapter 1 presents the introduction to NDT and highlights its importance in the assessment of concrete and timber structural members, and lists the objectives of this report. Chapter 2 describes the background, advantages, limitations, and applications of Ultrasonic Testing for condition assessment of structural members by providing a general overview of the process. Chapter 3, Chapter 4, and Chapter 5 present different reviews and testing methods of Ultrasonic Testing on materials such as concrete, steel, and timber structures. Chapter 6 presents the conclusions of the literature review and recommendations for future implementation. All the references cited in this report have been listed at the end. 


\section{ULTRASONIC TESTING OVERVIEW}

\subsection{INTRODUCTION}

The knowledge of ultrasonics has been around since the late 1800's, but the application towards NDT was not discovered until radar was introduced during the second world war. At this time, the process was very basic compared to today's methods. Ultrasonic testing was highly inspired by the sonar technique, sending sound waves through water to detect a submerged object (Blitz, 1996). Machinery became easier to attain, and less complicated to use in a field situation. The advancement in computer technology provides ultrasonics new challenges since most of the equipment relies on this machinery. With the progressive upgrades, the equipment has been developed to be smaller, faster, more compatible, etc. Ultrasonic testing is one of the most common modes of testing in not only the nondestructive field, but also the realm of condition assessment in civil infrastructure.

The ultrasonic technique is not limited to the field of civil engineering, but rather plays a role in many other fields of expertise. The medical field captured the importance of these nondestructive testing methods and evolved them into their own field. Some of the common uses for ultrasonics in other fields include surgery, dental treatments, chemical processing, etc. Figure 2.1 below, shows the slight variations in equipment and changing the frequency sound allows for other applications to be used such as the ones state above. Each of these applications allows a field to better understand the subject being tested promoting an easier lifestyle for years to come. 
Since these tests do not harm a subject, living or non-living, ultrasonics are a viable option for many areas such as the medical field.

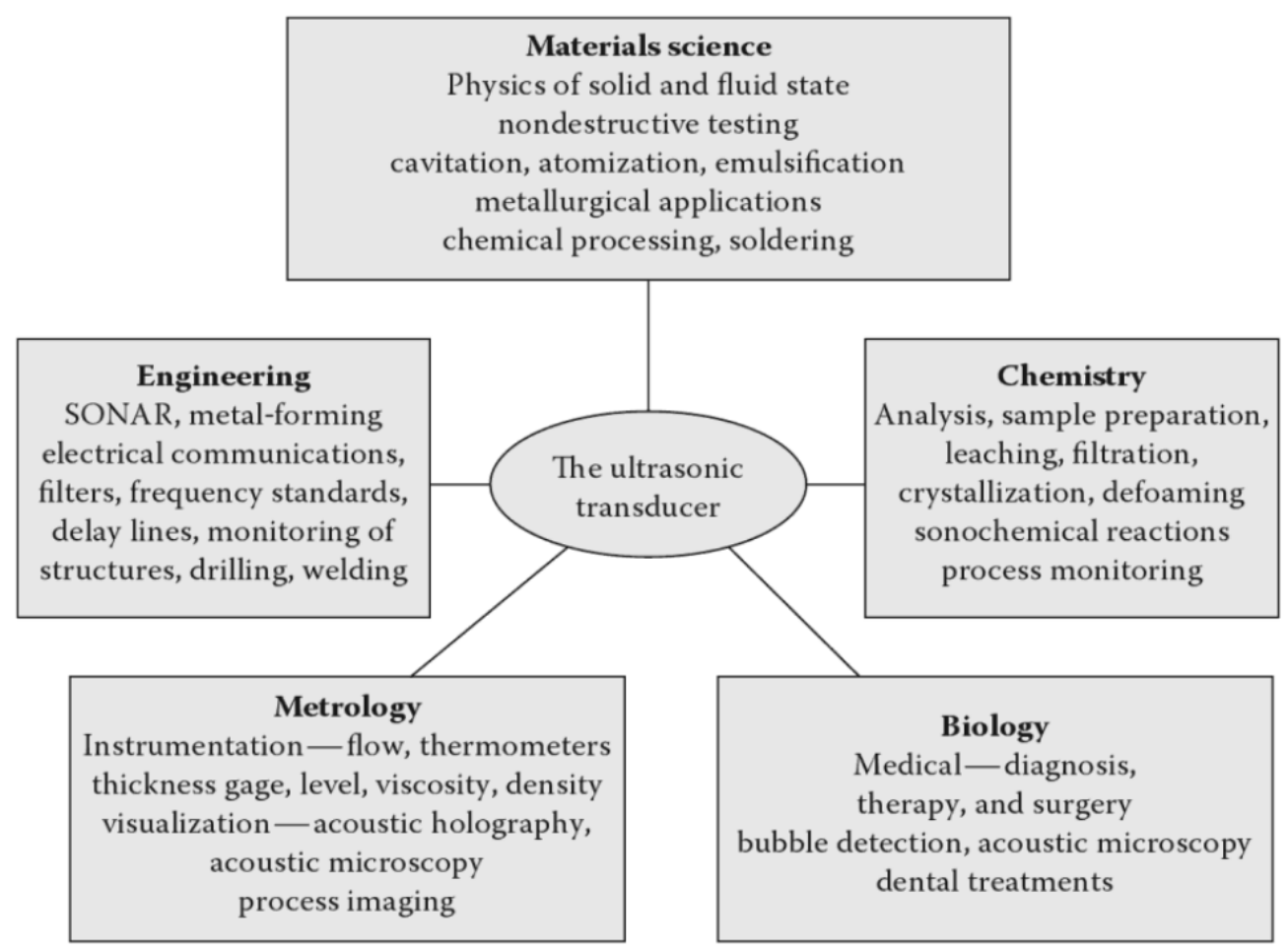

Figure 2.1: Ultrasonic transducer applications in other fields. (Stephens, 1975)

The applications of ultrasonic testing are just scratching the surface of the possible abilities. The more advanced technology evolves, the greater the range in science can be affected. This report will focus on the advancements and capabilities in the condition assessment for civil infrastructure rather than other science fields.

Ultrasonic testing uses high frequency sound (greater than $20 \mathrm{kHz}$ ), which the human ear does not have the capability to process. The range for the start of ultrasonic sounds can be taken as $16-20 \mathrm{kHz}$ depending on the health and state of hearing in a person (Ensminger \& Bond, 2012). The sound wave travels through the material under inspection and is able to detect any flaws or alterations in the structure or characterize the material for other measurements. Most equipment used in ultrasonic NDT consists of standard data-acquisition display devices, transducers, and a pulse system. These pieces may be altered or other add-ons are applied depending on the system 
and the user. Short pulses are produced through a pulse generator and directed into the transducer on the system. Waves at high speeds (high frequency) are generated from the transducer bringing forth the ultrasonic energy. The transferred energy in the system looks for breaks in the continuity of the medium. If the interacting waves detect a discontinuity, they reflect slightly (change directions) depending on the acoustic impedance. The reflected wave is transformed back into an electrical signal by the transducer device. Transducers will be applied on both sides of the member for several tests, but single sided access is needed for methods such as the pulse-echo method. This is highly beneficial for members that have limited access and only one surface is available. Once the signal transforms, the data displays on the data-acquisition display device. This data can easily be comprehended and evaluated by the user making the testing a viable solution for condition assessment.

Ultrasonic waves occur in different patterns and amplitudes based on the test being performed and equipment used. Longitudinal waves commonly occur in the system where the tests are being conducted in a longer body using short waves and high frequencies. Longitudinal waves may also be indicated as 'P-Waves' or pressure waves. Inside a member, when waves are bouncing around on the inside the pattern will be tighter on the excited side. Figure 2.2 shows the direction of the waves and the pattern with excited zones and the wavelength.

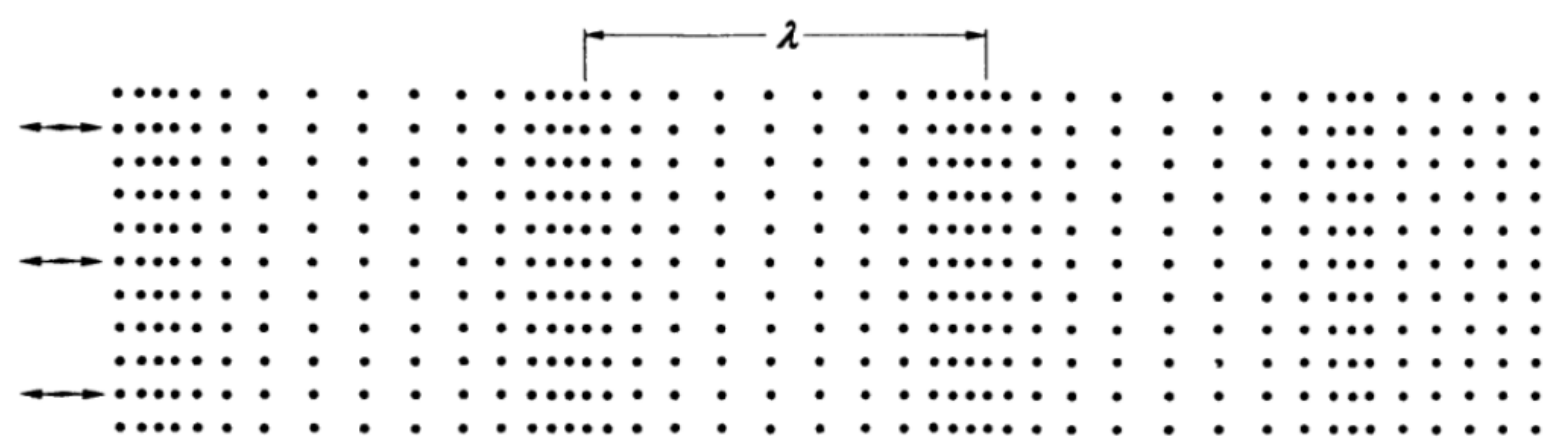

Direction of propagation $\longrightarrow$

Figure 2.2: Longitudinal waves showing excited zones. (Krautkrämer et al. 2013)

These zones are considered compression zones and occur as the wave travels though the body. Longitudinal waves oscillate in the direction of the propagation. Transverse waves are another 
occurrence of wave within a member which are shown in Figure 2.3. Transverse waves occur perpendicular to the member rather than in the same plane as the P-Waves travel. These transverse waves are also considered shear waves or 'S-Waves' and are conducted at right angles of the propagation.

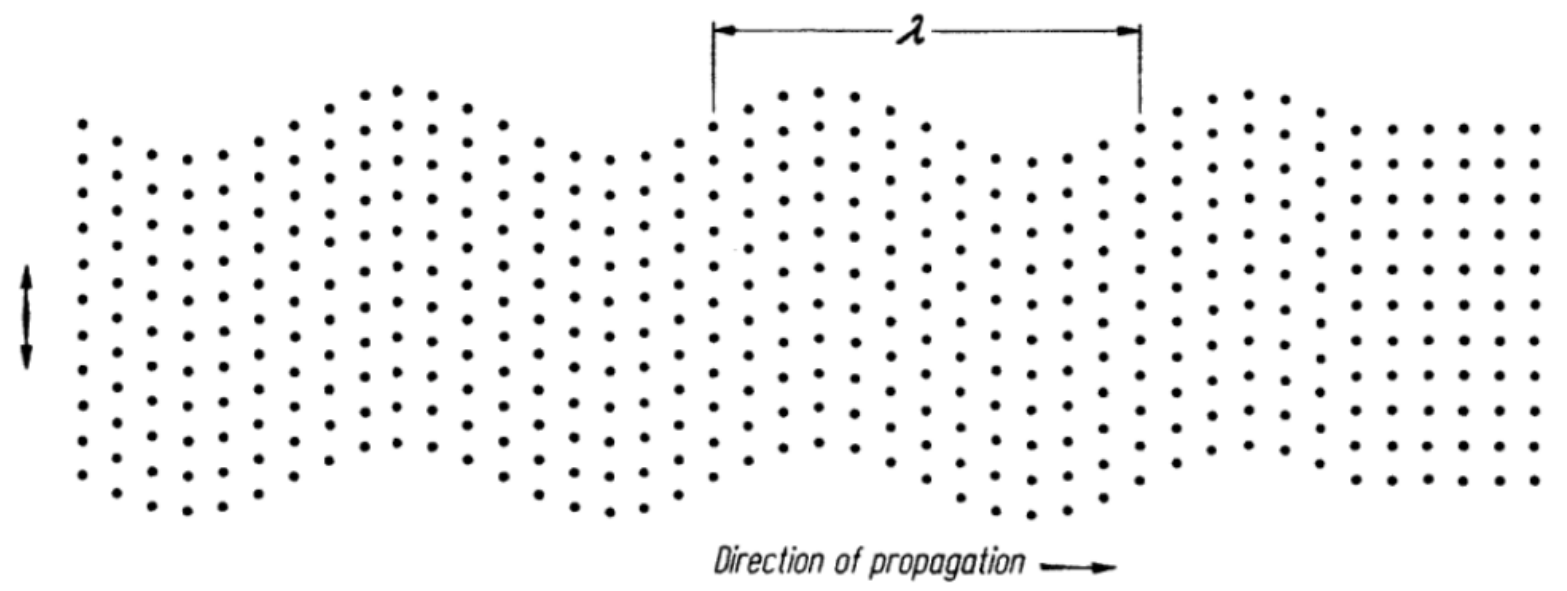

Figure 2.3: Transverse or Shear (S-waves) from left to right (Krautkrämer et al. 2013)

Other waves include plate waves (Lamb waves) and Rayleigh waves (R-waves). R-waves and plate waves are suited for examining small cracks or defects at the surface since the waves are propagating parallel to the boundary surface. On the contrast to pressure waves, R-waves and plate waves emit low frequencies since the test is conducted at one wavelength depth on average. Pipes and plates are commonly associated with these surface waves since they travel in the horizontal direction. Another beneficial factor for surface waves relates to a guided wavelength path that allows these waves to travel without interruptions for several meters.

The type of wave used and method chosen depend heavily on the subject being tested. The material properties for concrete vary heavily compared to those of steel, timber, fiber reinforced polymers (FRP), and virtually any other material that can be used for infrastructure. The typical frequency range employed in steel is $500 \mathrm{kHz}$ to $50 \mathrm{MHz}$, whereas it is $20 \mathrm{kHz}$ to $500 \mathrm{kHz}$ in timber. In concrete, because of high attenuation, frequency range from $20-150 \mathrm{kHz}$ is more suitable (Bungey et al. 2006). Each range can be pushed to higher and lower spectrums based on the type of experiment and test being applied. In many cases, the frequency for concrete reaches 
all the way to $200 \mathrm{kHz}$. The path length and minimum traverse dimensions are key factors that are able to be looked up prior to conducting an experiment.

Physics plays a large role in the evaluation of ultrasonic testing. If a wave sent through several members at a set frequency, each consisting of different materials, the wave will travel to different depths in the medium because of the material properties. For example, waves sent through steel will reach much greater depths than the same frequency wave sent through a concrete member. In order to increase the depth in a concrete material, the power in the pulse machine needs to increase. Increasing the amplitude in the machinery can increase the power in the machine, but there are still limitations for the device. The crystal inside the device can only move back and forth at certain max cycle. Once the crystal moves too fast, the device will malfunction. Before conducting any test, these parameters need to be evaluated and carefully chosen to keep the results in the best scope.

\subsection{EQUIPMENT COMMONLY USED IN ULTRASONIC TESTING METHODS}

\subsubsection{TRANSDUCERS AND RECEIVERS}

Piezoelectric materials play a huge role in the use of ultrasonic testing methods. One of the most common ways to create the ultrasonic waves involves piezoelectric devices. The basic principle of a transducer converts one form of energy into another form as it passes through a medium. In the case of ultrasonic testing, the transducer takes the electrical energy and converts it into a mechanical vibration which causes the detection. In both cases, having one point of contact or two, once the wave has finished in the medium it will be converted back from a mechanical wave to an electrical wave so the receiver will accept it. Shown below, in

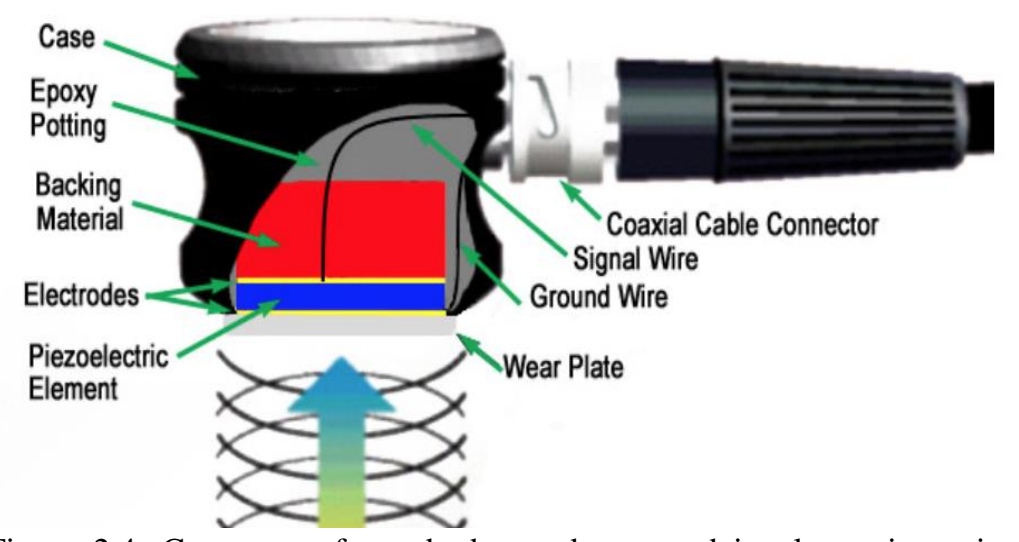

Figure 2.4: Cut away of standard transducer used in ultrasonic testing (Kishore, 2014) 
Figure 2.4 , is a typical cut away of a transducer. Transducers do not always need to touch the surface of the element to get the waves through the system. Most of the cases the transducer does make contact since it can lead to a smoother interface resulting in better information (Ryuzono et al. 2020). The one shown in the figure is a contact transducer.

Contact transducers contain extra layers compared to others to enhance the results and longevity of the equipment. These transducers are normally used when testing straight-beam elements for flaw detection or gaging thickness/other material properties. One of the reasons for the different setup relates to the sensitivity of the transducer. Different materials require lower sensitivity if the material penetration power increases. Contact transducers require an extra layer at the interface called a wear plate to protect the equipment from requiring any damage during testing. The size of the wear plate correlates to roughly $1 / 4$ the wavelength needed. When the wear plate meets this optimal range, it allows the waves to remain in the area rather than being lost. Based on the material being tested, an acoustical impedance may be required to alter the resistance to the material. Steel materials require a higher acoustical impedance due to the high density in the material and the resistance required to make it through the element. Healthcare workers require extremely different acoustic impedance since the material the waves travel through are typically bones rather than steel or concrete.

Angle beam transducers are another commonly used transducer typically found when a welding area is found on an element. Similar to a standard transducer, the energy is transferred from the system through the coupling system into the medium. Instead of the flat surface, the system us angled normally at 45, 60, or 70 degrees to generate the refracted shear waves (Michaels et al. 2006). Another common explanation for the device can be stated as a transducer placed on a simple wedge device. In some cases the devices are adjustable and the user must shift the transducer to the determined angle. The functions of the adjustable transducers may differ than the predetermined systems when it comes to the material that the test is being applied. For most cases, the fixed angle transducers deal with steel meaning that results will not be accurate if used on a different material. These angled transducers are commonly used in welded areas since the areas 


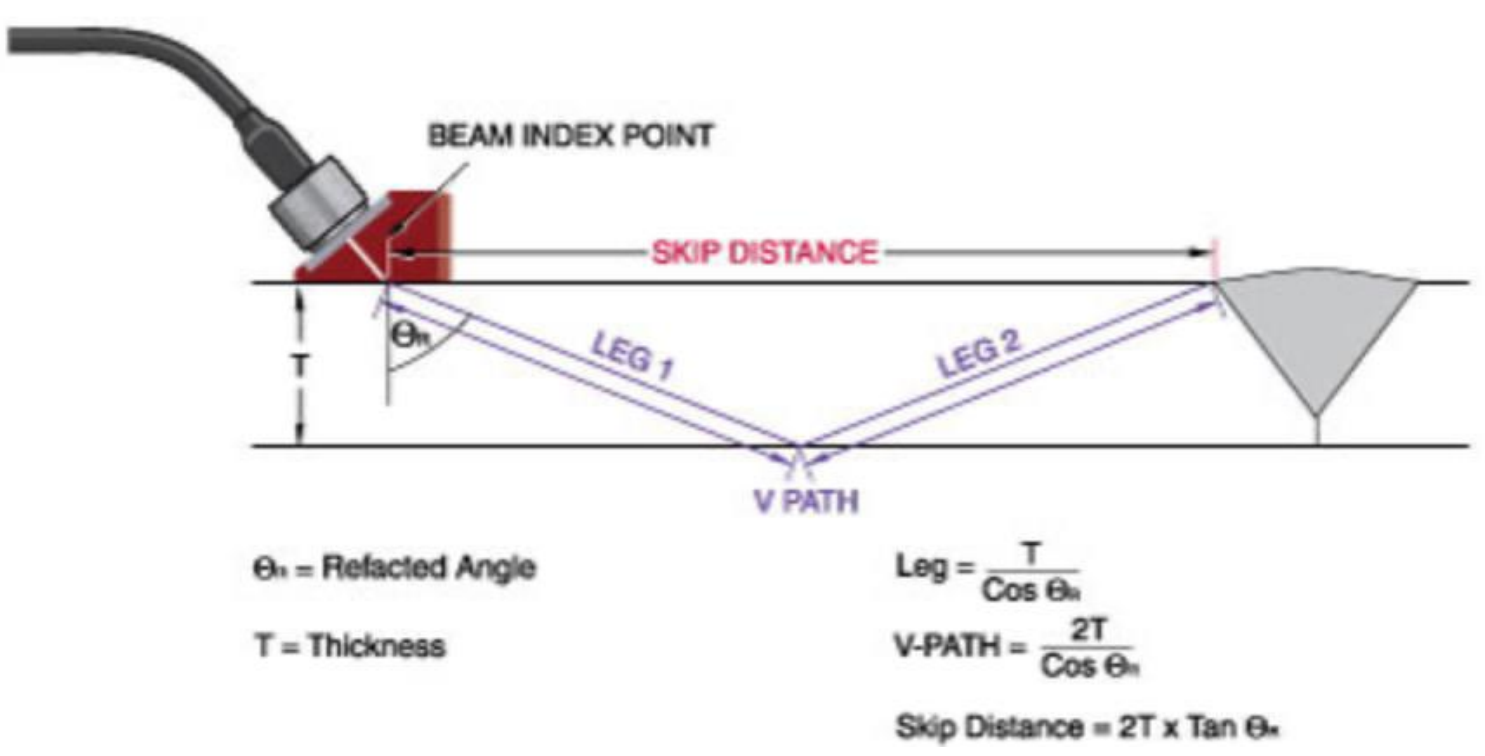

Figure 2.5: Angle Beam Transducer Position (Kishore, 2014)

By putting the transducer at an angle on the coupling surface it allows for the sound to travel a better path to detect flaws around the welded areas. Figure 2.5 shows the path taken to gets slightly underneath the altered area and the equations used to calculate the path. The skip distance will always be shorter than the actual path traveled by the wave since the sound rebounds off the back wall in the component. When the angle is zero the weld blocks the path, but the angle deflects the sound of the back wall covering the hidden area. Another commonly applied application for angle beam transducers are when surface waves are required to locate a defect. These surface waves find flaws and defects on the face of the system or the surface of the testing subject.

Performing a test using an angle beam transducer requires an experienced technician. Since using the regular equipment demands a skilled operator, setting the test at an angle increases the chances of locating insufficient results. Understanding where the waves are going to travel and how the information processes during the angled test needs an operator who has had practice in this field of testing. Since the inclination produces results characterized by high detail, the chances of having higher dead zones increases drastically.

There are other types of transducers such as delay line transducers and paint brush transducers and many more depending on the material being tested and the data required. If a material surface is 
relatively thin, the delay line method allows for the signal to be completed before it begins processing the results. When sending and processing happens at the same time, there can be a variation in results, hence making the delay line process extremely precise when gauging the thicknesses. While most of the transducers cover a smaller area for accurate testing, the paint brush transducer covers a larger area of the component. The widespread scan creates an effective way to scan for discontinuities over a wide spread, but other transducers or tests would be required to retrieve more detailed information

\subsubsection{COUPLING SYSTEMS}

In many cases, a coupling material sits at the interface between the transducer and the surface of the component to ensure a better connection with the acoustic impedance. Couplants are normally consisting of a liquid material that allows the energy to transmit into the testing material with greater ease. Without a coupling system in place, the difference in acoustic impedance between the outside and material (test subject) prevents the signal from being strong enough to record accurate data. When the connection between the two surfaces does not meet the required predetermined levels, a thin result is produced. Liquid materials are the common material used for the coupling system such as water, oil, even petroleum jelly techniques are applied as well as others. Other methods, such as submerging the entire transducer and the surface of the component into a water couplant. When trying to find precise measurements, the submersion method may be useful since the interface between the transducer, coupling system, and subject are seamless.

\subsection{TYPES OF ULTRASONIC TECHNIQUE (ACI 228.2R-13 )}

\section{$\underline{\text { Introduction }}$}

Multiple ultrasonic testing methods have been tested and used in the field of NDT to locate discontinuities or to measure material properties. The machinery has been altered and controlled differently depending on the scenario and price the user wishes to pay. The report gives a brief overview of some of the most common ultrasonic tests used in the laboratory or field testing. Other methods are available and may be commonly used depending on the scenario presented during testing. 


\section{Ultrasonic Pulse Velocity}

The ultrasonic pulse velocity observes the time of travel of ultrasonic pulses over a known distance. The attenuation of the ultrasonic pulses vary depending on the material and test being performed. The pulse velocity, or direct transmission, takes one of the fastest and accurate time readings for NDT or at least in the ultrasonic testing side of non-destructive testing.

a)

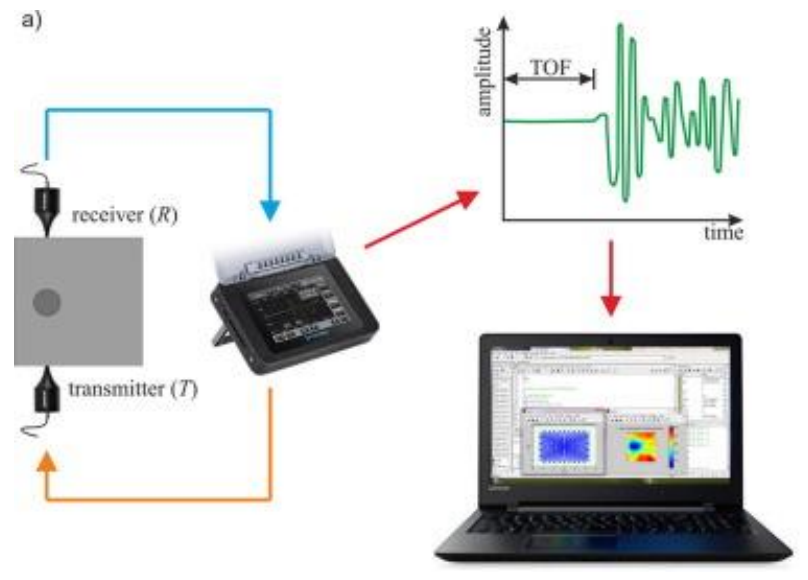

b)

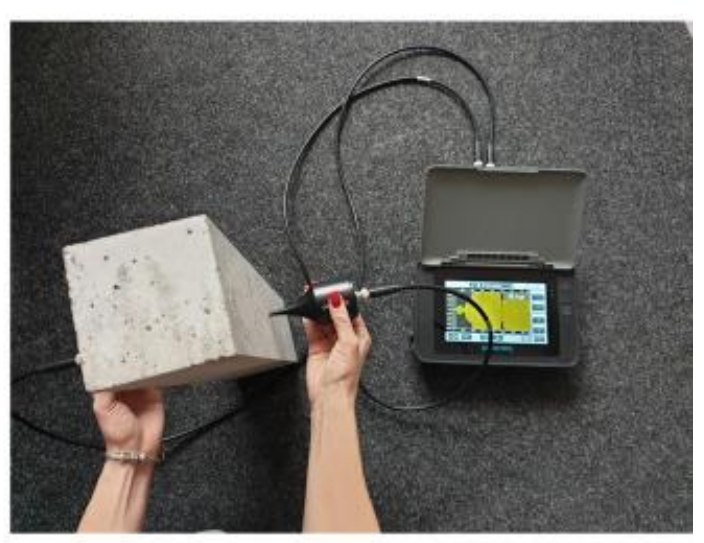

Figure 2.6: Ultrasonic Velocity test example and transducer setup (Zielinska \& Rucka, 2020)

The presence of defects and the effectiveness of repairs are able to be analysed by checking the quality of the test material. There are no dead zones when testing a subject, so a simple interpretation can be gathered from the data.

One of the limitations in the ultrasonic pulse velocity relates to the interface of the material. Both sides of the member need to be accessed in order to conduct the test since there would be a transducer on each side of the material surface as shown in figure 2.6 above. The surfaces must also be flat and parallel so the signal can travel through the specimen and record accurate results. When recording the data for the defects in the system, the pulse velocity test relays the information of a void or crack, but cannot locate the actual depth (Knab et al. 1983). The test locates voids perpendicular to the system, while parallel defects slip past the scan. 


\section{Ultrasonic Pulse Echo}

A single transducer measures reflected ultrasonic waves caused by discontinuities in the

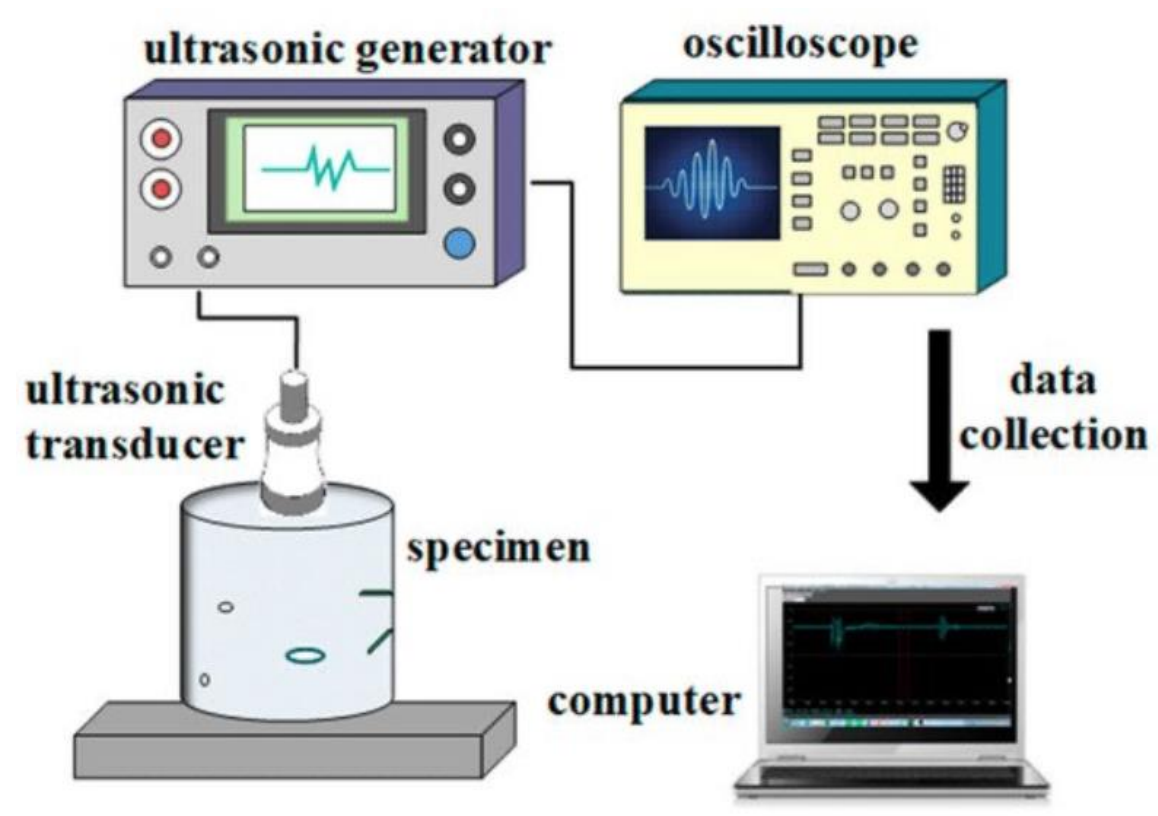

Figure 2.7:Example of ultrasonic pulse echo method setup (Tian et al. 2019) specimen or the backwall of the test subject. The transducer is capable of transmitting and receiving the data from the reflected waves. One of the key features in the pulse echo method allows the member thickness to be measured (Lipa et al. 2019). This can be great if the depth is not known before starting the initial investigation of the ultrasonic testing. Not only does the pulse echo method find the member thickness, the depth, size, and shape of defects are capable of being located. The wave travels through the member at a certain frequency where the distance can be calculated by recording the time travelled (Chirp Microsystems, 2020). Since the equipment only needs access to one side, the rest of the setup takes little to no time meaning the test can be used in a slightly mobile setting.

Just like the ultrasonic pulse velocity tests, the pulse echo method locates defects perpendicular to the wave direction. Waves travel over the defects parallel to the transducer direction and are incapable of detecting any flaws. The setup for the pulse echo method is simple, but an experienced technician runs the equipment. One of the key features for ultrasonic pulse echo relates to the transducer only needing one side of access for the testing to proceed (Wronkowics et al. 2018). Once understanding the way the equipment works the process becomes simple, but until training on the method takes place the method can be a challenge. 


\section{Angled Beam Assembly}

The angle beam assembly was referenced earlier in the report where the transducers are set at an incline using wedges. The waves travel at this angle offset to locate defects in a tilted position with respect to the testing surface. The angled beam method generates P-waves, S-waves, and Rwaves, but the ideal testing focuses on the shear direction for surface flaws (Lerch, 1996). The wave length for the angled setup increases compared to the direct setup since the wave bounces at the surface across the system rather than down and back. The setup requires an experienced operator since the angle changes the way direction path for the waves. A slight miscalculation or change in angle would cause the results to be skewed. The inclination also produces high resolution and can locate defects in different areas compared to the direct transmission, but the dead zones are much greater.

\section{Phased Array Method}

Multiple transducers and receivers are arranged in a linear pattern and act independently in the system. Each of the transducers are capable of sending signals through the beam at different time periods using an electronic delay to eventually collect all the information once each has returned a signal. All the transducers and receivers compile the information into a digitized piece of equipment and generate a tomographic image that relays real time analyse of discontinuities. The phased array method allows for accurate defect detection and high speeds by the combined power of the transducers and computer software available (Maes, 2018).

The major offset for the phased array method relates to the cost of the machinery and equipment. Since there are multiple transducers and receivers being used together, the price compared to direct transmission or other NDT is far greater. The method also needs greater software power so the A-scan signal can be completed to show the discontinuities.

\subsection{ADVANTAGES OF ULTRASONIC METHOD}

Ultrasonic testing provides several beneficial measurements that cannot be achieved using other methods of testing. Just like other NDT methods, ultrasonics allow the member to be tested for discontinuities and flaws on the surface and internally in the member. Other methods would only be able to provide one of these applications. Compared to other nondestructive methods, 
ultrasonic testing's depth of penetration is far greater for flaw detection. More accurate measurements at deeper depths provide users to spend less when conducting examinations. Instantaneous results provided by the equipment on site reduces cost and time when conducting the exams because wait time does not apply. Sending results to a lab and outsourcing the conducted work means paying another company for results, and waiting for those results to return. Construction and civil infrastructure rely heavily on time constraints as most projects have strict deadlines in a fast pace world. Ultrasonic testing has other functions outside of flaw detection that add extra benefit to the method. Being able to measure the thickness of a member alleviates the struggle going through other tests to determine the thickness (Wolf et al. 2014). Producing digital images of the member on the electronic automated systems provides an exclusive design concept that gives extra information including how to calculate thickness measurements. The wave travels through the member and peaks once the back wall is hit. Since the speed of the sound wave is known, the peak at the back wall and the return peak at the transducer origin provides enough data to calculate the distance traveled. The thickness is simply half the distance traveled since the back wall distance is the only thing needed.

\subsection{DISADVANTAGES OF ULTRASONIC METHOD}

Although ultrasonic testing contains a massive upside, like every test, there are also limitations to the testing method. Using the equipment provides a challenging task, especially to those that have never conducted tests similar to ultrasonics. Time and money devoted to training new technicians on the ultrasonic equipment proves to be slightly higher than other more basic methods. Once the training completes, the equipment functions in at an elevated level compared to other models. Being a skilled and proficient technician is required to initially start with ultrasonics. The surface face of the material has advantages and disadvantages. As suggested previously, the machinery needs to be connected to the surface from only one side in some cases. This can also be a disadvantage compared to other methods since a surface is required to perform the examination. Some other methods allow the test to take place without needing a connection to a face of the test product. Since the transducer connects to the face of the material, the interface between the two objects may interfere with the actual test. For many cases, the interface needs to have some type of coupling medium to assist in the transfer of sound. When the transducer bumps up against the surface of the material, the friction between the objects dilutes the sound waves into 
the test subject. The interface of the coupling medium will be discussed later in the report in more detail for each of the methods. For some tests, if the waves are traveling in a vertical direction and the flaw is in a horizontal direction it makes it easy to detect the defect in the system, but if the defect is in the same direction as the wave there is the slight chance that it could be missed or not fully recognized (Song et. al, 2020). The more paths of rays increases the range of detecting the defects and provide a better understanding of the material.

Ultrasonic testing provides an excellent method of results for many materials such as concrete, steel, and timber, but also has its material limitations. Although cast iron is not as common as some of the other materials, it can still be found being used in the modern day. Since cast iron consists of coarse materials, the sound transmission between the interface is not sufficient. The high frequency trying to insert itself into the material cannot pass through or provide accurate measurements. Going along with the material standpoint of testing, if the material has a rough surface or an irregular shape, the coupling medium does not properly transfer the sound waves through the material skewing the data collected. 


\section{CONDITION ASSESSMENT IN CONCRETE MATERIALS}

\subsection{INTRODUCTION}

Concrete can be seen in many of the existing structures since the material holds value extremely well compared to others and the cost. Most of the structures made using concrete have a design life of 50-100 years. The need for improvement in condition assessment has increased drastically since a large portion of the current concrete structures are right around lower end of the spread, 50 years. Large structures such as bridges are vital to maintain the adequate structural condition since they are traveled heavily by humans in everyday life. If a structure of this magnitude were to fail, the damage would be devasting and potentially fatal to a large group of nearby travelers. Bridges are located all over the world, and nearly one quarter of the structures in the United States needs some type of repair (Omar \& Nehdi, 2018). The inspection of bridges locates all of the repairs needed across the country, but there are other structures that have not been looked at, increasing the actual number of damaged bridges. Although these structures contain concrete, steel, and other materials, the more common area for potential damage remains in the bridge deck where concrete is heavily utilized. For the known bridges needing repair, nearly $\$ 70$ billion US dollars would need to be applied to ensure that structural repairs are met.

Just like any material, concrete has its own disadvantages that are typically known going into construction. Shrinkage, creep, voids in the surface are just some of the common defects found within concrete. Weather deals large amounts of damage throughout all structures and can be especially fatal to concrete depending on the state that the structures are currently in at the time. If voids and other cracks are present already, water seeping into the structure will severely damage the internal sections.

Reinforced concrete is the main component in the process since it is the material that is being tested using the ultrasonic method. Although concrete is such a strong material and has the potential to have a long life span, it also is susceptible to deteriorate from a multitude of things. Adding the reinforcing bars to the concrete increases both the strength and life span, but can also had extra areas that are able to be damaged. The bonding between the reinforcement and the 
concrete is one of the harder components to accomplish since the chemical makeup between the two needs to be correct. If the bonding does not work then there is a chance for the structure to crack and lose all potential strength (Rucka, 2018). The mixture of concrete needs to be manipulated in order to account for the reinforcing member being bonded. The most common reinforcing agent in infrastructure happens to be steel rebar members, but substitutes for steel would be wires, composites, or any other grids systems that could be placed within the concrete mixture (Zielińska,2020).

Limiting the reinforced concrete's deterioration is a high priority in infrastructure because of the damage that can follow. These mishaps can be physical or chemical damage, and each have several different ways to form. Typical visual signs for physical damage consist of cracks, spalling, and other forms of flaking or damage that can be seen from the surface. These damages are caused by everyday events that cannot always be prevented. Overloading the system is the most common of the physical process. An example of an overloading system would be an oversized truck running across a bridge deck. There is a system in place to set a weight limit on the structure, but trucks do not always follow this rule causing an extra weight to run over the surface. Other physical damages could be caused by fire depending on how intense and deep the effects reach in the concrete. Erosion is another common physical trait that goes hand-in-hand with abrasion that removes materials exposing internal concrete/rebar. Another environmental factor that cannot be avoided is when the weather drops below freezing. The water that seeped into the voids on the surface freeze within the system and expand causing cracking from within. The chemical damages are caused in a handful of ways, but usually result in the same damage type (Słoński et. al, 2020). Corrosion of the rebar has proved to be one of the most difficult and complicated situations to prevent in reinforced concrete. These effects cause incredible damage and sometimes result in failure of the system since the strength is reduced immensely. The corrosion calls for immediate repair of some sort which usually consists of wrapping the system in some other fiber.

When inspecting the concrete elements, defects can be seen on the external surface of the concrete if they are severe enough. If the damage is smaller than the eye can see on a large surface, that is where the ultrasonics would be able to aid in detection. These defects not only exist externally in the elements, but also internally where the human eye is incapable of viewing. Both the external and internal defects are capable of altering the structures service life and 
induce other unwanted damage. These defects can be seen through voids in the surface, spalling, shrinkage cracking, etc. along with other defects not mentioned.

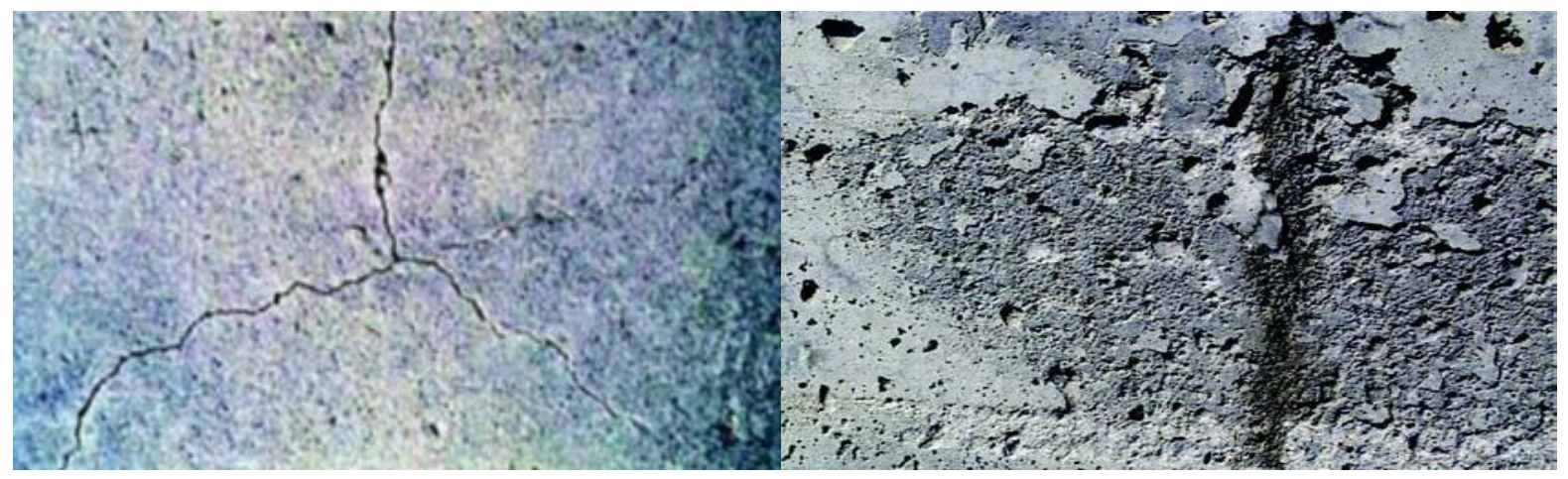

Figure 3.1: Example of spalling and cracking in concrete samples (Roblero, 2017)

In order to prevent these structures from having major defects in the system, inspections are performed regularly to ensure the design life of the structural element stays intact. The testing's are also performed for other reasons besides searching for the initial defects within the system. If regulations are not followed and the curing process is not run exactly how it should, the element would need looked at for any flaws. Any concern with the structural integrity, including the capacity allowed, requires extra attention (Juncai \&Hai, 2019). Other parameters to inspect the elements are considered, it is up to the discretion of those in charge to decide when. The table below illustrates some of the other common defects found in concrete materials from the physical and chemical issues.

Table 3.1: Common deterioration methods with supporting information (Breysse, 2010).

\begin{tabular}{|c|c|c|}
\hline Mechanism & Consequences in concrete & \multicolumn{1}{c|}{ Required Information } \\
\hline $\begin{array}{c}\text { Overloading, } \\
\text { Restraining effects } \\
\text { (temperature, } \\
\text { Shrinkage) }\end{array}$ & Damage, cracking & $\bullet \quad \begin{array}{l}\text { If distributed damage: crack } \\
\text { residual stiffness and strength } \\
\text { If localized cracking: location } \\
\text { depth }\end{array}$ \\
\hline Freeze-thaw cycles & $\begin{array}{c}\text { Scaling, spalling, } \\
\text { delamination }\end{array}$ & $\begin{array}{l}\text { Affected areas } \\
\text { Depth of defect }\end{array}$ \\
\hline Fire & $\begin{array}{c}\text { Strength decrease, } \\
\text { delamination }\end{array}$ & $\bullet \quad$ Depth Reached by fire effects \\
\hline
\end{tabular}




\begin{tabular}{|c|c|c|}
\hline & & $\begin{array}{l}\text { - Residual strengths at different } \\
\text { depths }\end{array}$ \\
\hline Abrasion-erosion & Material loss & $\begin{array}{l}\text { - Residual strength of surface } \\
\text { location }\end{array}$ \\
\hline Carbonation & $\begin{array}{l}\text { Increase in density, } \\
\text { depassivation of steel, thus, } \\
\text { rebar correosion }\end{array}$ & $\begin{array}{l}\text { - Carbonation depth } \\
\text { - If corrosion: localization of active } \\
\text { corrosion areas and corrosion rate }\end{array}$ \\
\hline Chloride attack & Rebar corrosion & $\begin{array}{l}\text { - Chloride content, chloride profile } \\
\text { - If corrosion: localization of active } \\
\text { corrosion areas and corrosion rate }\end{array}$ \\
\hline $\begin{array}{l}\text { Alkali-aggregate } \\
\text { reaction } \\
\text { Sulphate attack }\end{array}$ & $\begin{array}{l}\text { Internal expansion, } \\
\text { generalized cracking }\end{array}$ & $\begin{array}{l}\text { - Potential for future volume change } \\
\text { - Residual stiffness and strength }\end{array}$ \\
\hline Leaching & $\begin{array}{l}\text { Cement paste dissolution, } \\
\text { increase in porosity }\end{array}$ & $\begin{array}{l}\text { - Residual strength } \\
\text { - Porosity }\end{array}$ \\
\hline $\begin{array}{l}\text { Ammonium nitrate } \\
\text { attack }\end{array}$ & $\begin{array}{l}\text { Deterioration of cement } \\
\text { paste, spalling, rebar } \\
\text { corrosion }\end{array}$ & $\begin{array}{l}\text { - Depth of the attack } \\
\text { - If corrosion: localization of active } \\
\text { corrosion areas and corrosion rate }\end{array}$ \\
\hline
\end{tabular}

Different tests have commonly been used in the past to assess the condition and state of concrete structures, but the tests may have caused slight destruction. Several methods for concrete testing include fully destructive, semi-destructive, and then the newer method that evaluates without any damage being caused. 


\subsection{REVIEW OF APPLICATIONS OF ULTRASONIC TECHNIQUES IN DIFFERENT CONCRETE MATERIALS}

\subsubsection{PREDICTION OF REINFORCED CONCRETE STRENGTH BY ULTRASONIC VELOCITIES (SABBAG \& UYANIK, 2017)}

\section{$\underline{\text { Introduction }}$}

Measuring of the travel time of P-waves and S-waves through concrete structures instead of uniaxial compression tests can limit the damage done to a structure (Bompan \& Haach, 2018). Rather than collecting a sample from an existing concrete material, the ultrasonic test can be applied directly to the system without harming the structure. Since most concrete structures consist of steel rebar, samples with and without steel are examined. Steel rebar is placed into the concrete to increase the strength and stiffness of the material (along with other material properties), but it can also be harmful to the structure over time (Omar et al. 2018).

Rebar in concrete can be the eventual downfall of the structure due to processes over time. One of the largest concerns in structures can be from the damage of corrosion in the steel. Corrosion along with bad bonds between materials are reasons for continual testing of structures (Güclüer, 2020). 4 different types of cubes were crafted for the experiment, where some held rebar and others had no reinforcement. A number of studies were carried out on a total of 324 samples providing a large sample size for more accurate results. The cubes were also tested for uniaxial compressive strength by crushing the members. This gives a comparison between the two methods, and difference in measurements and values can be compared.

\section{$\underline{\text { Experimental Tests }}$}

The ultrasonic method of pulse velocity is used to determine the properties of concrete in the experiment using P-waves and S-waves. Since the distance is known in the experiment, the tome of travel of ultrasonic pulses can be observed using this method. The method provides the fastest and most accurate time readings with an extremely low error rate (Bucur, 2006). For the longitudinal direction, a grease or oil was used to eliminate the air gap between the transducer probes and the sample material. The shear direction did not have any extra element applied because the shear waves do not spread effectively in liquids. Some of the features that were sought after in 
the experiment include: sample rate, pulse data length, probe frequency, pulse amplitude. Other information including cracks in the system or voids undetectable to the human eye are evaluated in the sample as well. The pulse velocity method can provide the elastic properties of the concrete once the velocities in the longitudinal and shear directions are acquired thanks to the elasticity theory.

The samples were also evaluated for the uniaxial compressive strength so that the values could be compared. Even though this method is destructive, it provides the standard way of testing for these properties before the idea of ultrasonics. The cubes

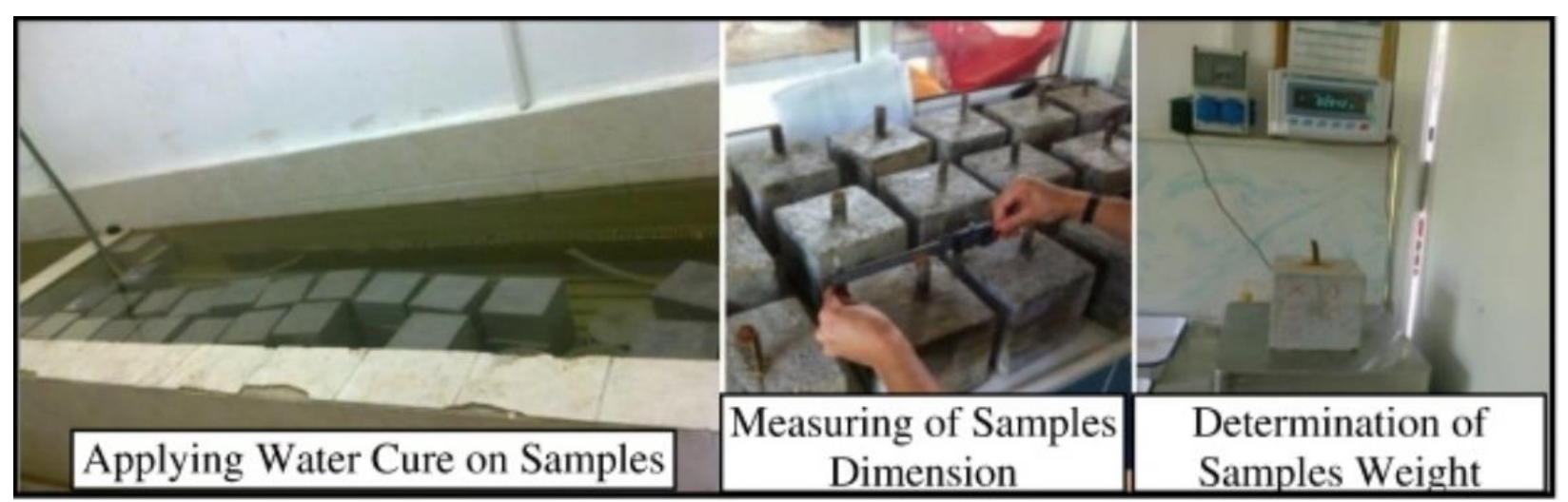

Figure 3.2: Demonstration of sample cubes being evaluated for testing (Sabbag \& Uyanik, 2017)

are placed on the compression test machine and a load is applied onto the cube between the two steel plates. The surface of the plates need to be clear of any debris so the load can be distributed evenly across the face of the sample. The compressive strength can be determined by the load at which the concrete fails divided by the cross-sectional area or P/A. The tests for uniaxial compression are typically done on a concrete mixture that had been allowed to sit for the 28 day curing period. The cubes need to be placed into a curing period, in this experiment the cubes were placed into a pool of water as shown in figure 3.2 above. Properties such as water content can be determined by dry weights and saturated weights from the curing pool.

Crushed stone aggregate was used in the concrete mixture for this experiment by using a stone crusher to get the predetermined size. Once the mixture was completed the cubes were made, each cube had a size of $150 \mathrm{~mm}$ cubed. Nine different water/cement ratios were used to create different strengths of concrete which included strengths of 5, 10, 15, 20 40, 50, 70, 80, and 100 $\mathrm{MPa}$. The cubes were split into 243 samples with varying size of rebar included and 81 cubes with 
no reinforcement. Of the reinforced samples, the cubes were split even farther where the size of the rebar varied between $10 \mathrm{~mm}, 14 \mathrm{~mm}$, and $20 \mathrm{~mm}$. The operations were carried out on multiple days to allow the concrete to develop and change strengths. This operation is possible since the tests do not destroy the sample, rather send a wave through the medium. The tests for longitudinal and shear wave measurements were completed on the $7^{\text {th }}, 28^{\text {th }}, 41^{\text {st }}, 56^{\text {th }}, 65^{\text {th }}, 72^{\text {nd }}$, and $90^{\text {th }}$ days so that the differences in strength were able to be compared.

\section{$\underline{\text { Results }}$}

The forces were determined for each of the cases on the given day being evaluated all the way to the $90^{\text {th }}$ day where the strength does not change as much. The velocities were shown to have a much higher change between the early stages. Concrete reaches high strengths at an early period and slowly levels off after the 28 day period. Between the $28^{\text {th }}$ and $90^{\text {th }}$ days, the velocities still had a period of increase, just not as great as the first 28 day testing. The velocities also had different ranges between the high and low strength concretes. The high strength concretes velocities had lower ranges due to time dependencies compared to the lower strength concrete samples. In figure 3.4 below, the results for the ultrasonic waves for both $\mathrm{P}$ and $\mathrm{S}$-waves and the uniaxial compressive strength are shown for the $7^{\text {th }}, 28^{\text {th }}$, and $90^{\text {th }}$ days. The graphs show each size of rebar in the sample including the samples without the reinforcing element. 

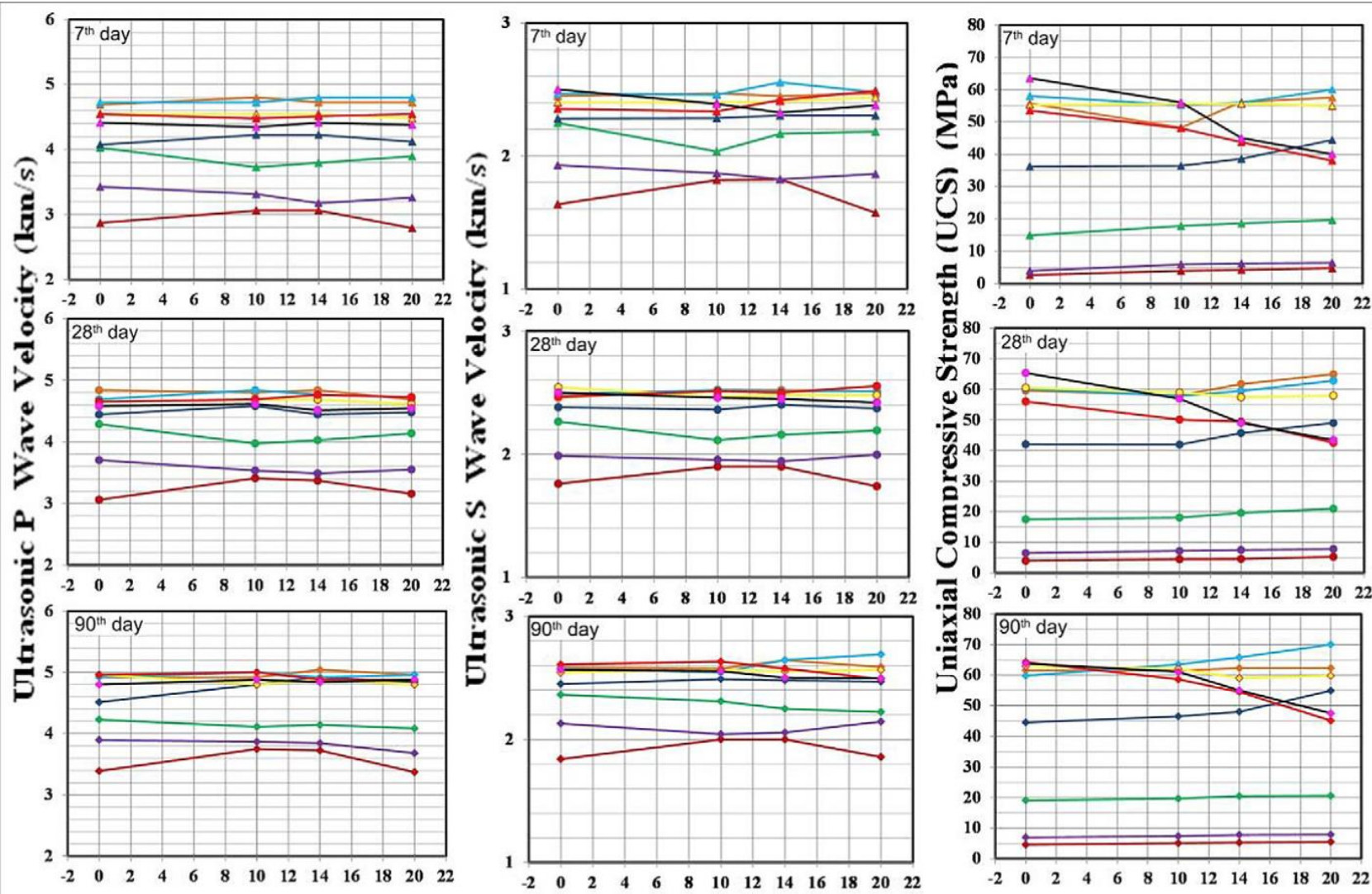

Reinforcement Diameter (mm)

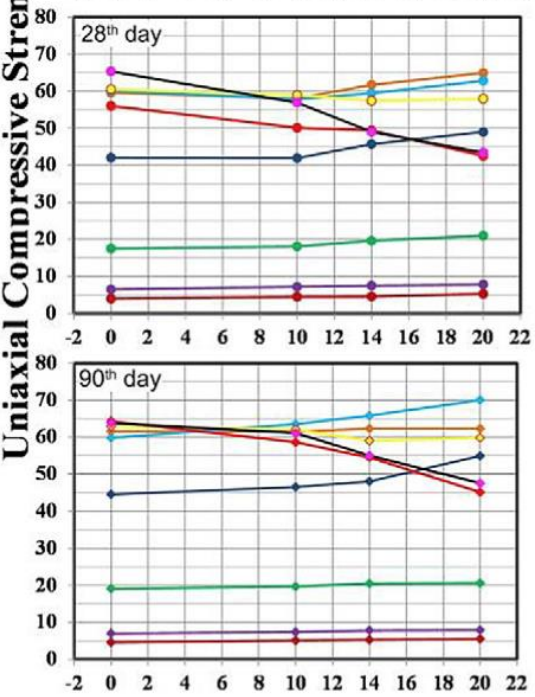

$\bullet \mathrm{D} 1 \bullet \mathrm{D} 2 \bullet \mathrm{D} 3 \bullet \mathrm{D} 4 \bullet \mathrm{D} 5 \bullet \mathrm{D} 6 \circ \mathrm{D} 7 \bullet \mathrm{D} 8 \bullet \mathrm{D} 9$

Figure 3.3: Comparison charts for P-wave velocity, S-wave velocity, and Uniaxial Compressive Strength with different reinforcing diameters (Sabbag \& Uyanik, 2017)

The figure above shows that the tests gained and lost strength differently between the 90 days. Some of the designs increased constantly, while others increased between the first 28 days then dropped in velocity. Factors such as the bond between concrete and rebar in the designs could be a reason for the differences in designs. The uniaxial compression strength shows the low levels between the $1^{\text {st }}$ and $3^{\text {rd }}$ designs, and the minimal change between the diameters of the reinforcing element throughout the 90 day period.

\section{Conclusion}

Ultrasonic velocity is a useful tool to determine the longitudinal and shear wave velocities in different elements and can compare the different levels of reinforcing members. High strength concretes and weak concretes react differently to the size of rebar placed within the member. The velocities increase in weak concretes when a larger piece of rebar lies in the system, while the 
higher strength mixtures is not affected in a positive or negative manner. Continual testing for strengths should continue for the advancement of knowledge in this field.

\subsubsection{ULTRASONIC TESTING OF REACTIVE POWDER CONCRETE (WASHER ET}

\section{AL. 2004)}

\section{$\underline{\text { Introduction }}$}

Standard concrete has been around for many years and has been one of the staples in the development of buildings across the globe. The makeup of concrete varies slightly between different mixtures, but always contains the same key ingredients: water, cement paste, aggregates, and other admixtures. The aggregates in concrete vary in size ranging from coarse aggregates and fine aggregates such as gravel and sands (Velay-Lizancos et al. 2018). The typical frequency for standard concrete materials ranges from $20-150 \mathrm{kHz}$ depending on the member being tested. Introducing a newer material, reactive powder concrete, the ultrasonic testing inspections can reach all the way to $1 \mathrm{MHz}$. In the powder material, there are no large aggregates such as the coarse pieces of gravel in traditional concrete. One of the largest aggregates in the reactive powder concrete can be seen as sand. Table 3.2 shows the mix proportions the mixture used in the experiment for the reactive powder concrete.

Table 3.2: Reactive Powder Concrete Mixture Sample (Washer et al. 2004 )

\begin{tabular}{|c|c|c|}
\hline Material & Amount $\left(\mathrm{kg} / \mathrm{m}^{3}\right)$ & Percent by Weight \\
\hline Portland Cement & 712 & 28.7 \\
\hline Fine Sand & 1020 & 41.1 \\
\hline Silica Fume & 231 & 8.3 \\
\hline Ground Quartz & 211 & 0.5 \\
\hline Superplasticizer & 13 & \\
\hline
\end{tabular}




\begin{tabular}{|c|c|c|}
\hline Steel Fibers & 160 & 6.4 \\
\hline Water & 136 & 5.5 \\
\hline
\end{tabular}

The lack of the large aggregates changes the attenuation in the material and allows for these higher frequencies. Since traditional concrete usually contains a steel rebar member to reinforce the concrete strength, that adds an extra element that needs to be accounted for when testing. The reactive powder material contains small steel fibers in the mixture that contributes to the overall strength without the added rebar members. Depending on the scenario, the steel rebar still may be within the powder concrete, but it is not as common compared to the traditional concrete members.

Even with the limited tests being completed for the powder concrete, compressive strengths ranging from 200-800 MPa have been recorded. These compressive strengths are far greater than that of the traditional concrete which typically stay below the strength of $100 \mathrm{MPa}$. One of the key negative aspects of traditional concrete can be seen when testing the tensile strength of the material. While normal concrete has relatively low tensile strength, the reactive powder exhibits a much higher strength in the tensile direction. Since the powder contains the small fibers of steel within the mixture, it provides the extra strength that traditional concrete cannot.

\section{Experimental Tests}

Ultrasonic Pulse-Echo tests were setup to try to analyze and experiment on reactive powder concrete samples. The testing apparatus consisted of a compression spring that applied a constant inward pressure on the system. The other two parts of the system consisted of a transducer jig and the transducer itself. The jig along with the compressive swing provide a uniform contact pressure on the ultrasonic transducer. A high powered ultrasonic instrument was used to create the ultrasonic pulses. The system used was a RITEC RAM-10000 which created single-cycle pulses into the test subjects. Shear waves and longitudinal waves were both applied to the test subject using different coupling materials. For the shear wave test, a high viscosity coupling substance was applied. The shear wave coupling system was provided by the manufacturer, but the longitudinal utilized a petroleum jelly for the coupling. 
The tests for the reactive powder concrete were conducted on several distinctive samples (cubes, cylinders, prismatic member) that each provided a different means of information and results. The test specimen manufactured using steel fibers and without the steel fibers to get a range of results. The length of the cubes and the percent of steel in the specimen are shown in the table 3.3 below. The highest percentage of steel in the cube mixture was $2 \%$. Other mixes may have higher amounts of steel depending on the project the concrete will be applicable for whether it be a high traffic bridge or a low use concrete structure.

Table 3.3: RPC Cube lengths and percentage of steel fibers in mixture (Washer et al. 2004)

\begin{tabular}{|c|c|c|c|c|c|}
\hline Specimen & L1 (mm) & L2 (mm) & L3 (mm) & Density (kg/m3) & Steel Fibers (vo1\%) \\
\hline L3-17 & 100.01 & 97.66 & 99.94 & 2465 & 2 \\
\hline L3-18 & 99.95 & 97.96 & 99.94 & 2462 & 2 \\
\hline L3-19 & 99.85 & 97.79 & 99.92 & 2467 & 0 \\
\hline L12-3 & 99.89 & 99.74 & 99.90 & 2350 & 2 \\
\hline
\end{tabular}

The cubes containing the steel fibers were cured in a differed environment from the cubes without the fibers. The member without the steel fibers was steam cured, while the members containing steel were air cured in a laboratory environment. These cubes were used to get basic information for wave propagation.

Cylinders were the second set of samples being tested that would provide results for wave propagation through a shape that commonly gets tested when determining information in the field. Similar to the table above, the information regarding the cylinders can be found below in table 3.4. The split between specimen with steel fibers and without are 50/50 for the cylinder case. 
Table 3.4: Parameters for cylinders tested with steel fiber percentage (Washer et al. 2004)

\begin{tabular}{|c|c|c|c|c|}
\hline Specimen & $\begin{array}{c}\text { L1 } \\
(\mathrm{mm})\end{array}$ & $\begin{array}{c}\text { Diameters } \\
(\mathrm{mm}) \\
(\text { nominal })\end{array}$ & Density (kg/m3) & Steel Fibers (vol\%) \\
\hline L10-41 & 143.1 & 76.2 & 2491 & 2 \\
\hline L10-42 & 142.2 & 76.3 & 2484 & 0 \\
\hline L12-01 & 151.7 & 76.2 & 2360 & 0 \\
\hline L12-02 & 147.7 & 6.2 & 2351 & 2 \\
\hline
\end{tabular}

The last testing specimen was prismatic member that was given four cracks of variable lengths by loading the member. The two outside cracks only extended $15 \mathrm{~mm}$ into the member, while the interior cracks extended more than twice the exterior cracks, $35 \mathrm{~mm}$, into the specimen. Since the purpose of the experiment was to determine properties of the newer concrete and to examine if the reactive powder concrete was able to use ultrasonic pulse echo tests, there were a lot of uncertainties.

\section{$\underline{\text { Results }}$}

High test frequencies were able to be seen throughout the cubes through two cycles from multiple values. The first test conducted at $500 \mathrm{kHz}$ was easily able to detect these waves in the longitudinal and shear directions. The second test, conducted at $1 \mathrm{MHz}$, also provided strong results proving that reactive powder concrete was able to be evaluated at such a high frequency. A higher transducer set at $2.25 \mathrm{MHz}$ was sampled at a smaller capacity producing low signal-to-noise ratios. The signals were able to complete the entire round trip (down and back) which would be an extreme case for other elements. The farthest made crack on the beam was located 210mm away from the transducer, meaning the entire trip would be $420 \mathrm{~mm}$ long. Traditional concrete bridge decks are normally around the order of $200 \mathrm{~mm}$ thickness, which means that the ultrasonic pulse echo method is a viable method in these cases. 
Table 3.5: Longitudinal and Shear waves tested at $500 \mathrm{kHz}$ (Washer et al. 2004)

\begin{tabular}{ccccccc}
\hline Specimen & $\begin{array}{c}\text { Average } \\
\text { P-wave velocity } \\
(\mathrm{m} / \mathrm{s})\end{array}$ & \multicolumn{2}{c}{$\begin{array}{c}\text { Maximum, minimum, } \\
\text { and standard } \\
\text { deviation } \sigma(\mathrm{m} / \mathrm{s})\end{array}$} & $\begin{array}{c}\text { Average } \\
\text { S-wave velocity } \\
(\mathrm{m} / \mathrm{s})\end{array}$ & $\begin{array}{c}\text { Maximum, minimum, } \\
\text { and standard } \\
\text { deviation } \sigma(\mathrm{m} / \mathrm{s})\end{array}$ \\
\hline $\begin{array}{c}\text { Specimen } \\
\text { with }\end{array}$ & 4913 & Max. & 4937 & & Max. & 3084 \\
fibers & & Min. & 4894 & 3074 & Min. & 3059 \\
Specimen & & $\sigma$ & 10.5 & & $\sigma$ & 5.5 \\
without & 5031 & Max. & 5034 & & Max. & 3155 \\
fibers & & $\sigma$ & 5029 & 3147 & Min. & 3141 \\
\hline
\end{tabular}

Shown in table 3.5 above, demonstrates the results of the longitudinal and shear waves when tested at $500 \mathrm{kHz}$. The wave velocities can be seen for both the case with steel fibers in the mixture and without the steel fibers. The wave velocity without the steel fibers is shown to be higher in the longitudinal and shear directions. Comparing with the table that consists of the 500 $\mathrm{kHz}$ waves, table 3.6 below allows an insight on the samples with $1 \mathrm{MHz}$ applied. The cubes without the fibers again demonstrate a higher wave velocity for P-waves and S-waves.

Table 3.6: Longitudinal and Shear waves tested at $1 \mathrm{MHz}$ (Washer et al. 2004)

\begin{tabular}{ccccccc}
\hline Specimen & $\begin{array}{c}\text { Average } \\
\text { P-wave velocity } \\
(\mathrm{m} / \mathrm{s})\end{array}$ & \multicolumn{2}{c}{$\begin{array}{c}\text { Maximum, minimum, } \\
\text { and standard } \\
\text { deviation } \sigma(\mathrm{m} / \mathrm{s})\end{array}$} & $\begin{array}{c}\text { Average } \\
\text { S-wave velocity } \\
(\mathrm{m} / \mathrm{s})\end{array}$ & $\begin{array}{c}\text { Maximum, minimum, } \\
\text { and standard } \\
\text { deviation } \sigma(\mathrm{m} / \mathrm{s})\end{array}$ \\
\hline Cubes & & Max. & 4975 & & Max. & 3101 \\
with & 4948 & Min. & 4928 & 3089 & Min. & 3076 \\
fibers & & $\sigma$ & 12.2 & & $\sigma$ & 6.6 \\
Cubes & & Max. & 5077 & & Max. & 3173 \\
without & 5070 & Min. & 5059 & 3166 & Min. & 3158 \\
fibers & & $\sigma$ & 5.7 & & $\sigma$ & 4.1 \\
\hline
\end{tabular}

The cubes being tested had the most samples giving a much better base for future results, since error can be taken out slightly. Moving to the cylinders, table 3.7 below, demonstrates the results for the $500 \mathrm{kHz}$ and the $1 \mathrm{MHz}$ velocities in the longitudinal and shear directions respectfully. The cylinders without the fibers have a much closer velocity to the ones with steel 
fibers. This may be due to the small sample size for the cylinders compared to the multiple lengths tested in the cubes.

Table 3.7: Cylinder velocities in the P and S-waves with and without steel fibers (Washer et al. 2004)

\begin{tabular}{ccccc}
\hline Specimen & $\begin{array}{c}\text { Average P-wave } \\
\text { velocities } \\
(\mathrm{m} / \mathrm{s})\end{array}$ & \multicolumn{2}{c}{$\begin{array}{c}\text { Average S-wave } \\
\text { velocities } \\
(\mathrm{m} / \mathrm{s})\end{array}$} \\
\hline & $500 \mathrm{kHz}$ & $1 \mathrm{MHz}$ & $500 \mathrm{kHz}$ & $1 \mathrm{MHz}$ \\
\hline $\begin{array}{c}\text { Cylinders } \\
\text { with }\end{array}$ & 5043 & 5049 & 3146 & 3151 \\
$\begin{array}{c}\text { fibers } \\
\text { Cylinders } \\
\text { without } \\
\text { fibers }\end{array}$ & 5029 & 5033 & 3150 & 3160 \\
\hline
\end{tabular}

The velocities for the cylinders fluctuate between which mixture has the higher velocity. In the longitudinal direction the cylinders with the fibers contain the higher velocities, while the opposite is the case for the shear direction.

\section{$\underline{\text { Conclusion }}$}

Reactive powder concrete can be evaluated using the ultrasonic pulse echo method with continued research and development. Since this was a preliminary testing experiment using ultrasonics, more work will be needed to further evaluate the material and its properties. This method can also be determined able to test for cracks in the samples of reactive powder concrete. The ultrasonic technique of pulse echo can be used on the reactive powder concrete at least up to $400 \mathrm{~mm}$ due to the high density and other properties of material. In all of the tests scenarios the wave velocities in the longitudinal were reaching values around $5000 \mathrm{~m} / \mathrm{s}$, while the shear direction stayed around $3100 \mathrm{~m} / \mathrm{s}$ for each. The samples with the steel fibers produced velocities slightly lower than the samples without the steel. This may be caused by having another material present with different properties or the way the samples were cured. Continual research and evaluation would be an appropriate solution to advance the industry in a positive direction. 


\subsubsection{FAST AND REAL TIME INSPECTION OF CONCRETE STRUCTURES USING A ROLLING 3D SCANNER (CHAPAGAIN ET AL. 2018)}

\section{$\underline{\text { Introduction }}$}

This paper introduces a multilayered ultrasonic rolling scanner intended to assess the structural properties in concrete. The scanner consists of an eight-element transducer array units that utilizes a piezoelectric polyvinylidene fluoride (PVDF). The ultrasonic scanning array system was designed by ELOP to analyze defects in concrete structures at a frequency range of 50-250 $\mathrm{kHz}$. Since the system has multiple layers, it makes up for the low electro-mechanical coupling efficiency compared to the commercial ceramic transducers used in other systems. The
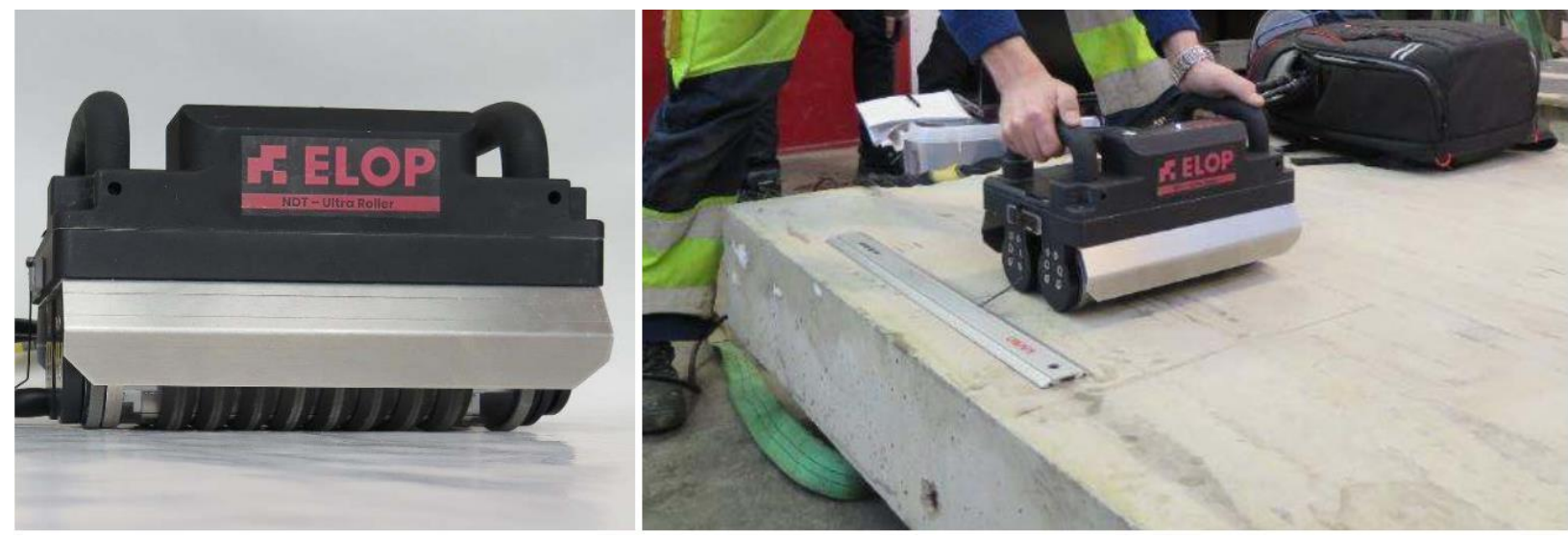

Figure 3.4: Multilayered Ultrasonic Rolling Scanner built by ELOP (Chapagain et al. 2018)

scanner consists of two separate rollers to analyze the concrete structures, one being the transducer (Tx) while the other being the receiver (Rx). Figure 3.4 shows the setup of the ultrasonic rolling scanner in detail. The rollers use elastomer rings as the coupling system to the surface of the concrete since the unit will be not be stationary. The electrical impedance helped achieve a higher sensitivity and a lower resonance frequency.

\section{Field Tests}

The layers in the multilayer transducer were accomplished by stacking the PVDF films in a sequence and holding them together using a adhesive. The frequency range was shifted closer to the optimal range typically associated when testing concrete inspection. The assembly was configured to best minimize the acoustic reflections between the layers. The actual electronics platform that sat on top of the scanner was designed and built in house for the experiment. The 
electronic system on top will transmit a signal into the concrete, trying to excite the subject. The scanner continuously rolls manually, sending acoustical reflections at the defects back into the receiver transducers for farther analyzation.

Since there are 8 transducers firing signals into the concrete structure, each receiver will receive these elements until they are all completed. 3D images are able to be created using a synthetic aperture focusing technique (SAFT) from the 64 A-scans that were collected from the Tx and Rx rollers. The imaging system was improved by including SAFT in the parallel direction and the perpendicular direction the scanner was rolling.

\section{$\underline{\text { Results }}$}

A concrete block, $20 \mathrm{~mm}$ thick, was analyzed for defects using the ultrasonic scanner in the along-track and cross-track direction. The blocks were prefabricated with defects before the tests were initiated to see if the scanner was capable of locating the air tubes or rebar inside. A 150 $\mathrm{kHz}$ frequency was used to scan the block at a $0.1 \mathrm{~cm}$ interval.

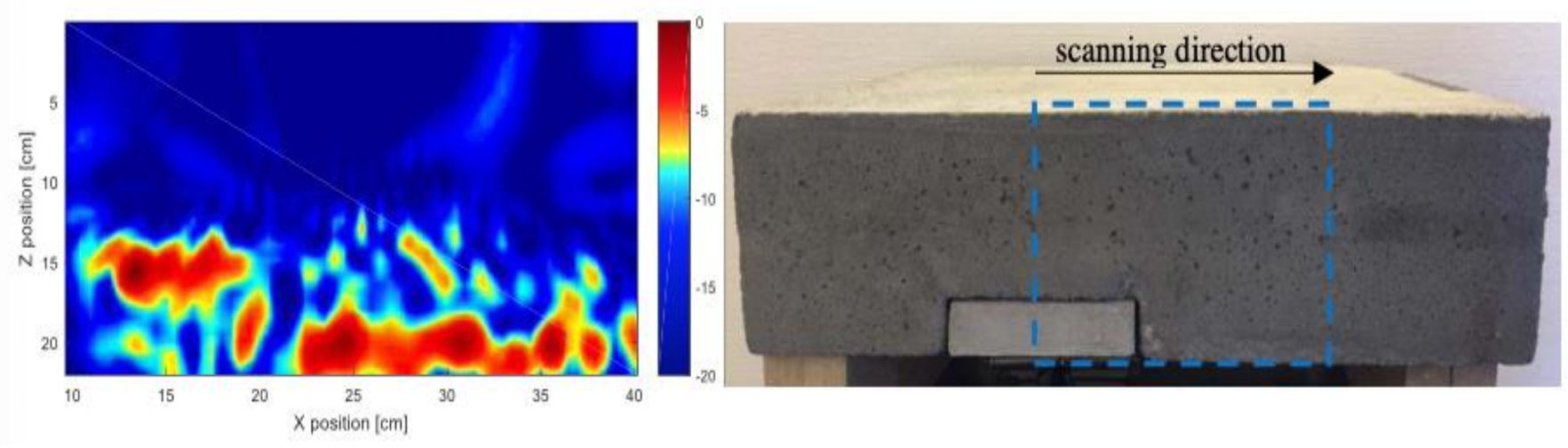

Figure 3.5: Example of $20 \mathrm{~mm}$ slab with a defect at a depth of $15 \mathrm{~cm}$ (Chapagain et al. 2018) 

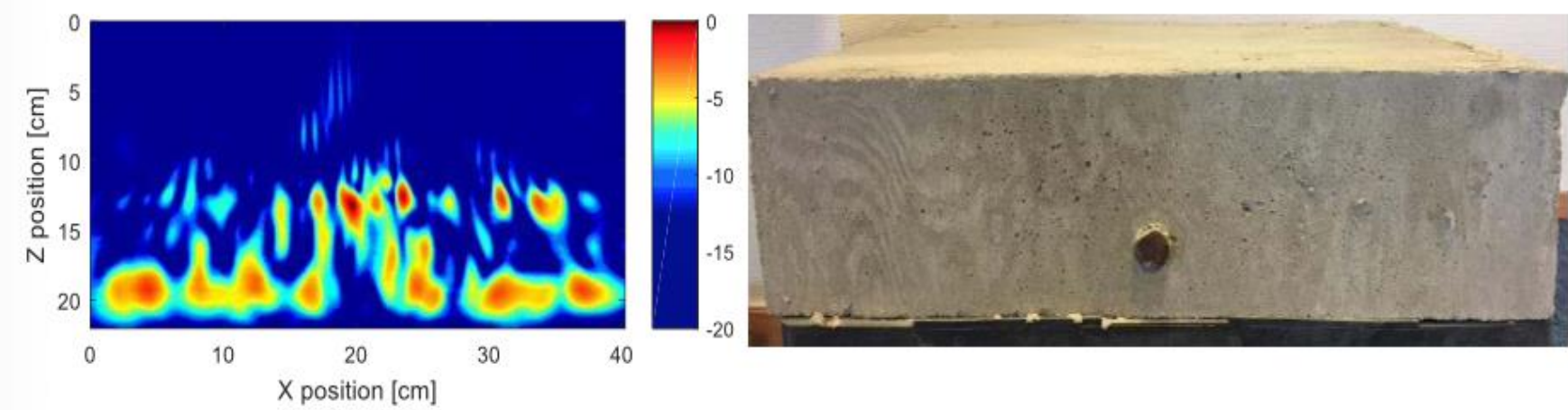

Figure 3.6: Concrete block with a steel rebar placed at $12.5 \mathrm{~cm}$ depth (Chapagain et al. 2018)

The blocks contained different sized aggregates that ranged from $8 \mathrm{~mm}$ to $22 \mathrm{~mm}$. The laboratory tests had delamination's and rebar at depths of $15 \mathrm{~cm}$ and $12.5 \mathrm{~cm}$ and were both visibly located once the scan was completed as shown in figures $3.5 \& 3.6$ above. The field experiment was also able to locate rebar at a depth of $13 \mathrm{~cm}$ from concrete containing different aggregate size and properties.

\section{$\underline{\text { Conclusion }}$}

Advancement and continued improvement will need to be completed on the scanner for more accurate and precise work. The scanner portrayed a unique way to use ultrasound for concrete inspection by developing a multilayer piezoelectric polyvinylidene fluoride array transducer that was capable of transmitting and receiving signals. The handheld device allowed a large area of concrete to be scanned for defects with quality imaging in a short time period. The future models will try to complete the scan without a lag between the real-time display and reduce the cost of labor since the machine will take less time.

\subsubsection{ULTRASONIC ASSESSMENT OF THE CONCRETE RESIDUAL STRENGTH AFTER A REAL FIRE EXPOSURE (WROBLEWSKI, R., \& STAWISKI, B., 2020). \\ Introduction}

Disasters occur in structures all the time so they are built to try and withstand whatever element may be present. Fires are one of the more common disasters on concrete since many buildings have a chance for this hazard to take place. When steel reinforcing member is present in the concrete structure that challenge between the bond occurs since both of the materials will act 
separately. The longer time exposed to the extreme temperatures strength and usefulness of the concrete will decrease. Some of the factors damaging the durability include moisture content involved in many of the mixtures (Teng et al. 2019). Once the temperature reaches a boiling point, the free water will begin to evaporate in the system. The concrete and steel both have different deformities when a thermal element is introduced and can easily shift when high temperatures are reached. With the rapid evaporation of water in the concrete, cracks begin to form throughout the system causing the strength to rapidly decrease and eventually fail. Damage due to fires are easy to assess from the human eye standpoint, but a more in depth analysis needs ultrasonic testing to deliver accurate strengths.

Color change in concrete can be an initial indicator after a fire for initial damage, but the strength cannot be determined if the cracks are covered or new cracks have formed. Figure 3.7 depicts an examples of the change in color on concrete after an exposure to fire revealing the rebar inside the member. The way the rebar has been exposed due to the fire in sample (a) is called concrete splitting. Sample (b) in the figure shows the change in color of concrete in a cylinder from top to bottom. Remains from other pieces melt and cover the concerning areas where cracks 
may be located. The loads are evaluated differently in design rather a real case scenario because of the different levels being presented and different temperatures that may be achieved.

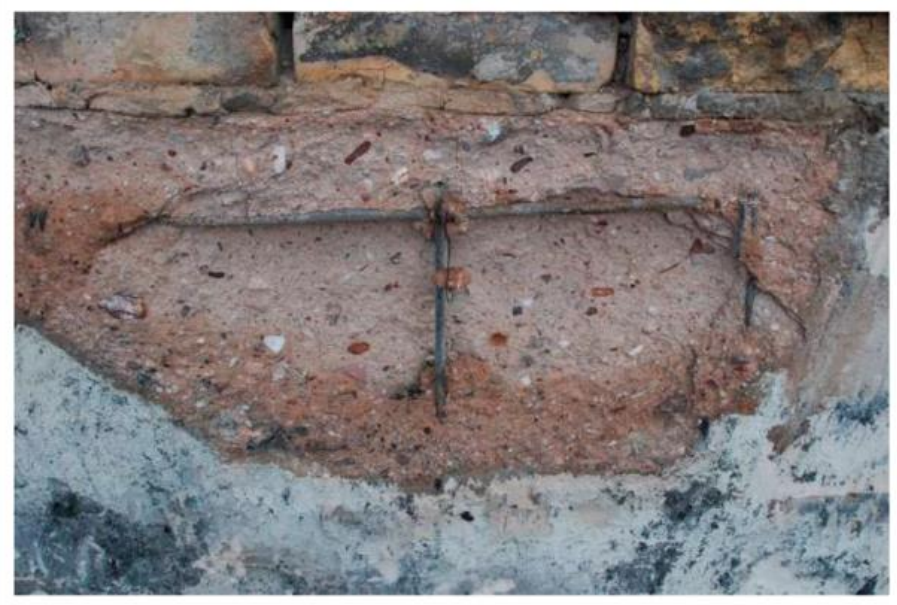

(a)

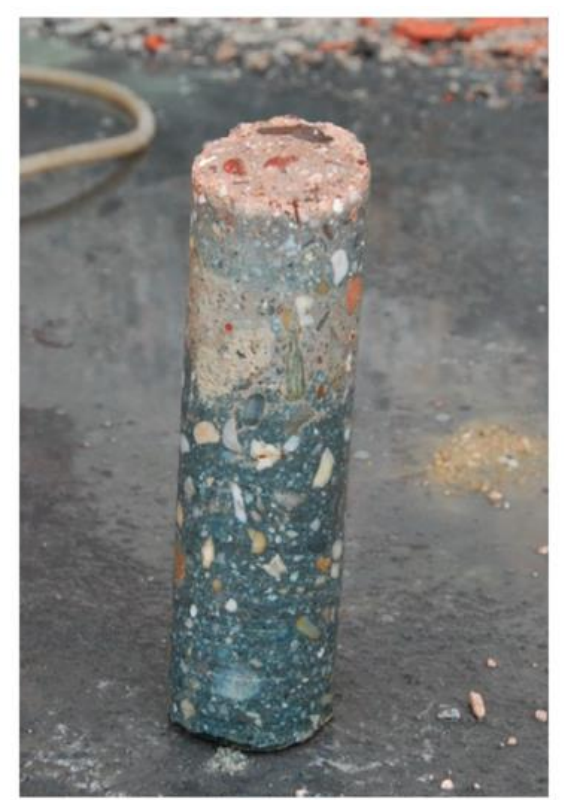

(b)

Figure 3.7: (a) Steel rebar exposure (b) color change after effects of fire exposure on core sample (Wroblewski \& Stawiski, 2020)

The strength in the structure not only needs to be analyzed during the actual disaster, but also post fire since the materials will continue to change once the cooling process starts again. In normal conditions a set of parameters can be predetermined assumptions can be assumed for material behavior. Once the conditions of the fire are present, these assumptions can no longer be made since the material properties will change with the extreme thermal fluctuation. Many of the post-fire tests are not conducted during a real fire with the fear of harming the structure more, rather these post-fire tests occur in simulated experiments. The results will also vary from experiment to real settings in large buildings due to travelling fire. In laboratory experiments, the fires are always controlled and directed where real fires take different directions and temperature levels depending on the spread. The open area in a building creates a change in element on its own since it creates a larger space. Known speeds and spread of the fire can be predicted in certain scenarios to evaluate the strength between the steel rebar and the concrete members including the bonds. It was determined that Ultrasonic Pulse Velocity methods would be used to analyze the 
concrete exposed to fire as long as it remains under 600 degrees Celsius. The elastic modulus and moisture content are the two main factors for the limitation due to temperature effect.

\section{Experimental Tests}

The case study was conducted based off a 64-apartment building that had been exposed to a fire while being constructed. Figure 3.8 below, shows the face of the building where the fire originated and was the strongest point. The weather during the fire was favored the spread ultimately allowing the fire to spread the whole building. The temperature, humidity, rain, and wind were all at appropriate levels that did not impeded the spread, but rather assist. The fire was aided by materials stored near the strongest area of exposure.

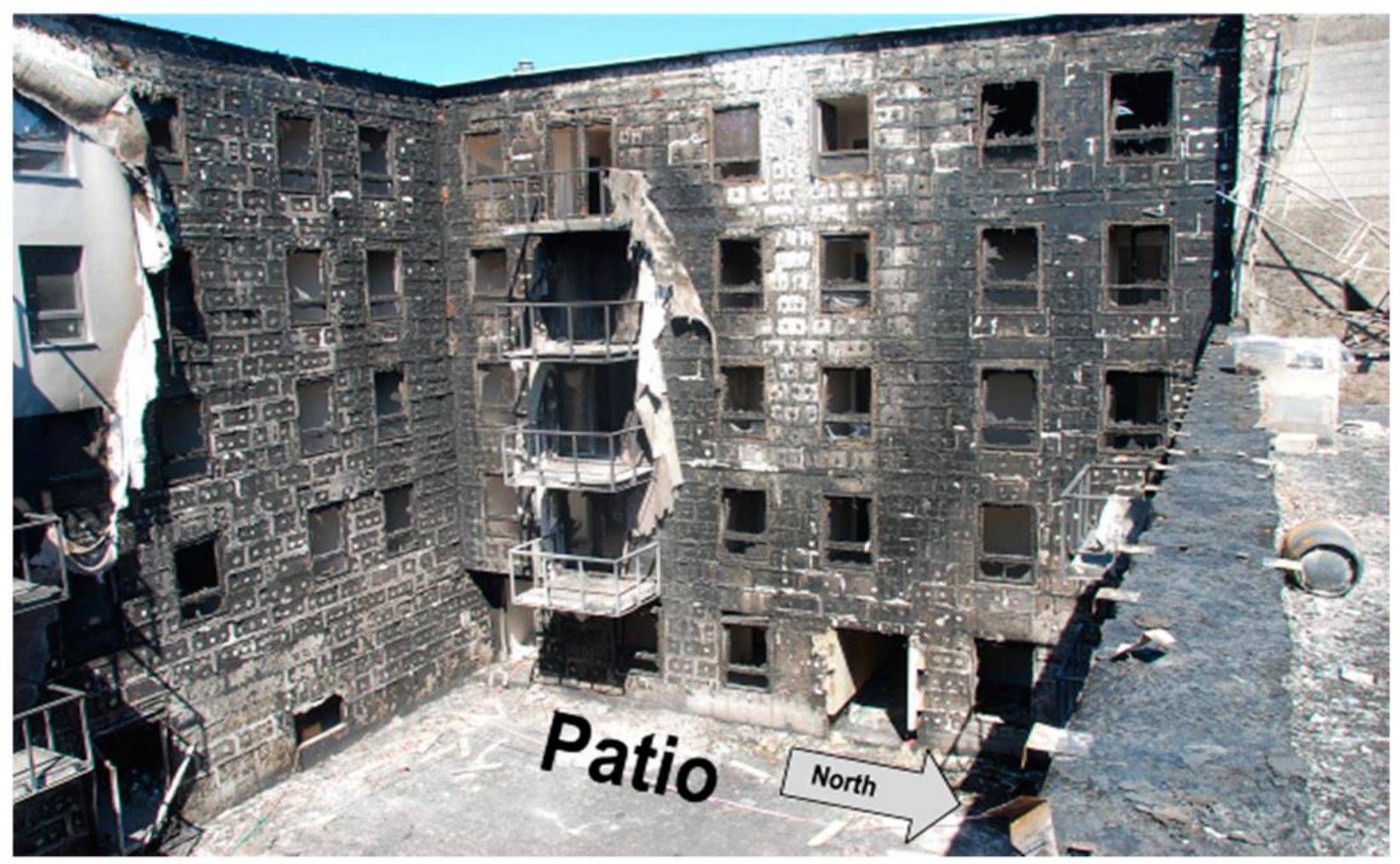

Figure 3.8: Face of 64-apartment complex building after exposure to fire (Wroblewski \& Stawiski, 2020)

Building materials such as Styrofoam and roofing paper aided in the rapid growth in temperature because they both burn at temperatures above 200 degrees Celsius. The damage on the building also was a result of chemicals burning off materials and being directly in contact with the concrete. 
Concrete core samples were taken from separate areas where the fire had spread so the temperature and strength of the concrete would have different levels (Wroblewski \& Stawiski, 2006). The cores were taken at depths where the strength had not changed due to the fire for safety reasons. Removing a core from a damaged area could have been detrimental to the worker retrieving the sample. The cores were split into different groups so that the in-situ compressive strengths could be tested using a uniaxial compressive strength machine. These destructive tests were conducted to ensure that the strength had not changed in the concrete so the results would have the same compressive strengths that were intended.

The machinery used for the ultrasonic test was a Unipan 543 digital device that had two point ultrasonic heads. The heads were able to produce $40 \mathrm{kHz}$ into the sample for testing. To make the testing seem more real, each sample was exposed to the fire for an unknown time and temperature. The core samples were placed into the apparatus

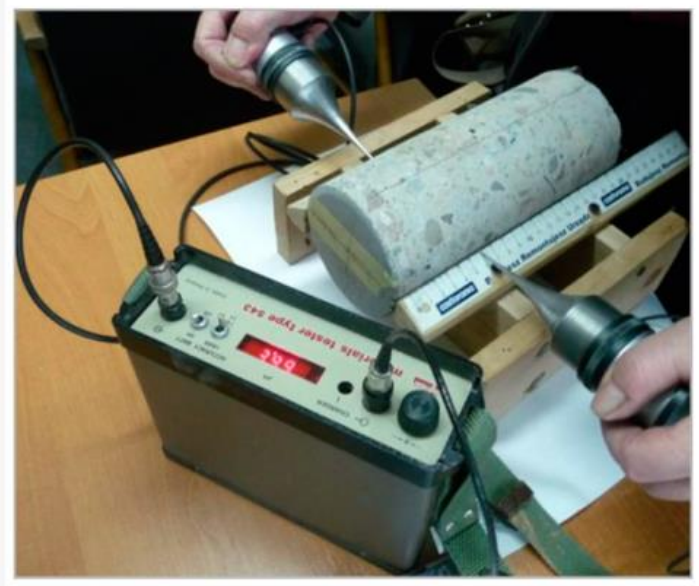

(a)

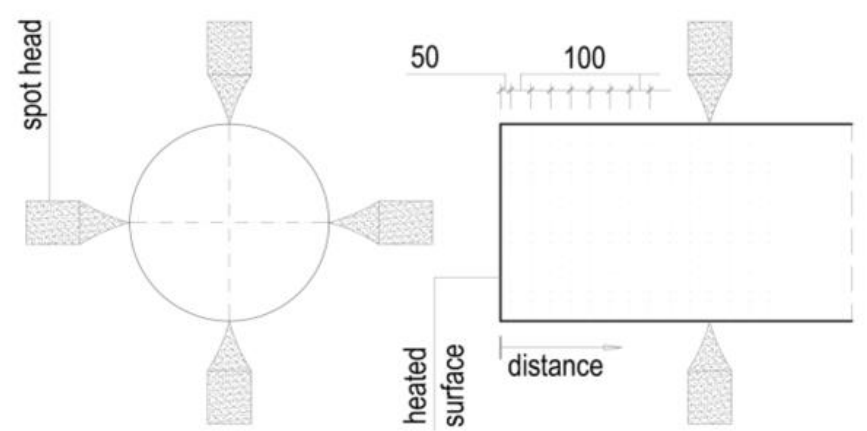

(b)

Figure 3.9: Example setup of Unipan 543 digital device and placement of ultrasonic point heads (Wroblewski \& Stawiski, 2020)

and the ultrasonic velocity was determined by taking the average of the two measurements in perpendicular directions. Figure 3.9 above depicts the setup given for the device and the placement of the point heads. Each of the cores for the experiment were placed in the device to get an accurate result from the samples. 


\section{$\underline{\text { Results }}$}

The strengths varied depending on the concrete sample since each was exposed to a different level of the fire and temperature. Throughout most of the concrete samples tested, the strength towards the surface of the concrete is roughly half that of the bottom layer. The middle section, between the top $25 \%$ and bottom $25 \%$, the strength of the concrete indicated that it was only $25 \%$ reduction from the bottom section of the layer. Not all of the samples followed the same pattern, but the numbers above reflect the vast majority of the samples taken. The change in the strength consistently jumped in strength starting right around $30 \mathrm{~mm}$ deep in the concrete samples. Since the jump in strength is so large, this area is considered the fire effect. Figure 3.10 shows the strengths of one of the sample cores over different increments of depth.

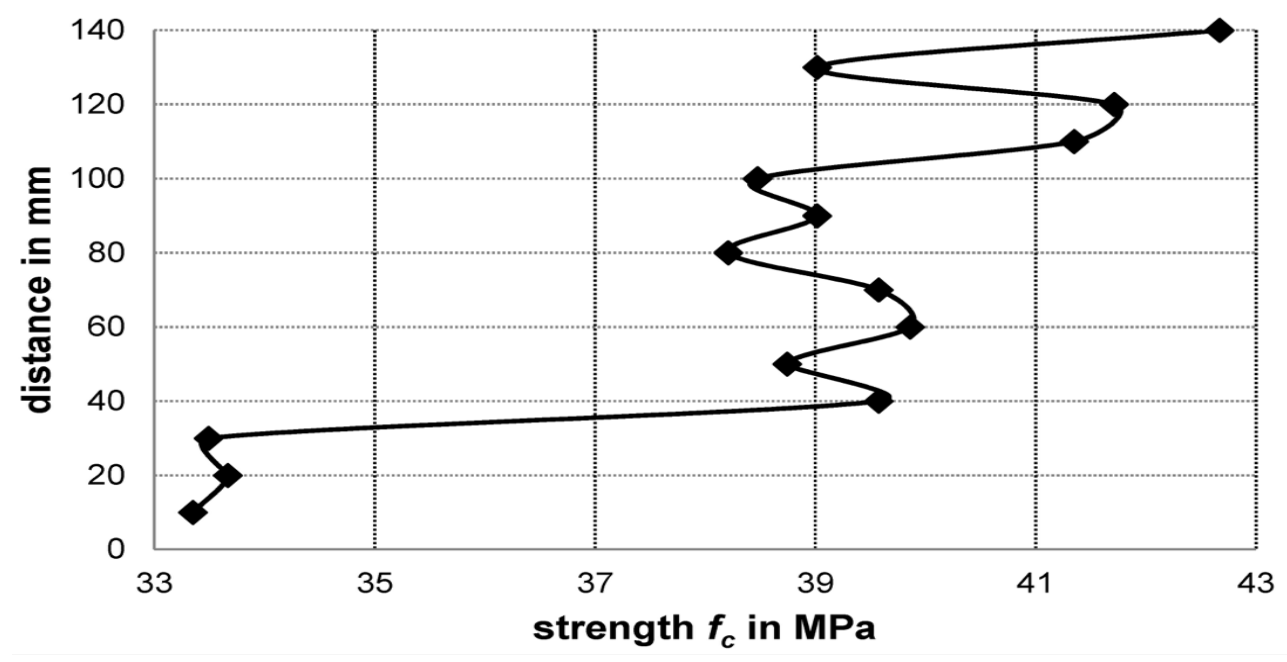

Figure 3.10: Strength of concrete sample after exposure to fire based on depth of testing (Wroblewski \& Stawiski, 2020)

The strength in the above example can be considered as natural effects since the decrease stays within the $25 \%$ range.

\section{Conclusions}

The test provided an accurate prediction of strength of concrete in different depths when exposed to fire. Structural elements, slabs, concrete members, etc. can all evaluated using the ultrasonic method. Since the samples were taken at longer distances to gauge the depth of effect, using a smaller sample size would provide more accurate results and better compressive strength values. Since the previous method was a visual examination, the ultrasonic method provides a 
better representation of the concrete strength. Visual inspection can become difficult to evaluate the actual safety of the structure based on color change.

Future work would require collecting another sample from the exposed/dangerous region of the building which can be one of the drawbacks of the testing. Most of the samples are taken from the visual inspection since these are typically the areas that would have the most damage, even if this is not the most accurate method. The ultrasonic tests cannot be conducted on their own to get an accurate reading, but need an accompanying destructive test to get results for unaffected areas so there is a value that can be compared. Farther research to find potential ways to eliminate the extra work are being conducted in the future. Once the destructive aspect of testing is removed from the process, the cost for completion can heavily rely on the usage of the NDT machinery and the technicians required to run the equipment. Other NDT testing techniques can be used outside of ultrasonics to get similar results. The case is the same for many other NDT techniques since the method is still relatively new compared to the existing tests that have had the time to validate themselves over the years.

\subsubsection{CONDITION ASSESSMENT OF CONCRETE ELEMENTS THROUGH TWO NONDESTRUCTIVE ULTRASONIC TECHNIQUES (ROBLERO, 2017)}

\section{Introduction}

A new method for the condition assessment of concrete columns was being introduced by applying two methods of ultrasonic testing. The ultrasonic wave velocity and wave attenuation measurements were combined to evaluate the damage in reinforced concrete columns. In many cases, using two methods achieve greater results rather than one method alone. One method of ultrasonic testing retrieves vital data for a member, but there will always be some measurement left out for the experiment. Applying the second method covers the disadvantages that the first test misses, so choosing a method with the right information can be imperative to record the correct results. Modifying equipment improves the data recorded if altered correctly, incorrectly and the system will provide negative results.

Since this specific equipment modification had never been tested prior to the experiment, one of the goals was to set a baseline set of results for future work to be done on the system. One 
of the ways to advance the field of NDT deals with pushing the boundaries and testing new equipment even if the process does not succeed on the first experiment.

\section{Experimental Tests (Ashbrook, 2021)}

In order to test the elements in the field, steps must be taken in the laboratory to prepare for everything. In the laboratory, several scenarios were set up to eliminate any weaknesses in the test. The first scenario was to set up an ultrasonic pulse velocity test to determine the compression and the freeze/thaw damage in a concrete element. Prisms were tested to ensure that the tests were accurate with one another and to determine if the test had to capability of detecting the fracturing internally in the concrete.

As stated earlier, these tests work best when two or more methods are combined to produce one final result. The second laboratory step was to set up and determine the surface waves in a given scenario. These tests were conducted to try and find the limitations in the method for the specific test case. It was determined that this method of NDT would be the best for the outer portion since energy on the surface is best suited for the method. It is also the easiest method to achieve the results for the project. If anything goes wrong on these laboratory tests, the setup can easily be modified to reach the ultimate setup and testing result. Even with the laboratory test and field test being different setups, it shows advantages and disadvantages in the test process.

The final part of the laboratory setup was constructing the field testing device known as an UTPole system that had a NI USB-6356 display device. This system consists of a software component, electronics component, and the transducers. The software component of the UTPole device ensure that the test runs properly and is able to process all of the data that needs collected. The electronic component contains the pulse generation system, amplifier, and the data acquisition system. The transducers for this test were able to reach a nominal frequency of $82 \mathrm{kHz}$. The UTPole system is useful when it comes to testing wood poles or reinforced concrete columns. Modifying the original system was a huge concern to ensure the test ran properly. Since the device would be in the field the electronic system needed to be modified so that it could be portable for field testing. The technology needed to be condensed to a portable size, while maintaining all the capabilities needed to run the test on concrete (Alyousef, 2016). The transducers also needed to be able to function in a reasonable time frame. Multiple configurations of the test are needed to 
provide an accurate sample, so having a quick test time aids in quicker results. A faster test time also provides the user to experience similar field conditions such as temperature change and loading situations. Adequate coupling of the transducers needed to be checked to make sure the elements were receiving the waves in the designed manner. Since the coupling is on the face of the element there is room for slippage leading to inaccurate results due to a lower level of pressure being applied on the waves.

\section{$\underline{\text { Results (Ashbrook, 2021) }}$}

When beginning to collect data in the field, it is imperative to understand the software that would be controlling the testing. In this case the UTPole software calculates the velocities of Pwaves in the given element. In the case of a cylindrical reinforced concrete section, the software breaks the section into 25 different sections and solves for the wave velocity for each path in the element. The system works in reverse to find the wave velocities by analyzing the output in order to find the input (wave velocities). In the concrete section, the test can be affected by the makeup of the mixture including the moisture content in the element. Moisture contents can increase the velocity in the system by $5 \%$ (Zamen \& Dehghan-Niri, 2020). When the concrete contains a reinforcing member the software must be able to compute the distances and materials inside the concrete. Since the steel members have different properties and higher acoustic impendence than the concrete surrounding, this in turn would provide a higher velocity for the internal member (Shokouhi et al. 2017). The tests were originally completed on a mortar cylinder to ensure the system worked properly. The mortar was chosen since the properties are closer to concrete than other materials would be giving the test verification that the coupling systems would work to the 
full extent. The two plots below demonstrate the velocity profiles for the given experiment.

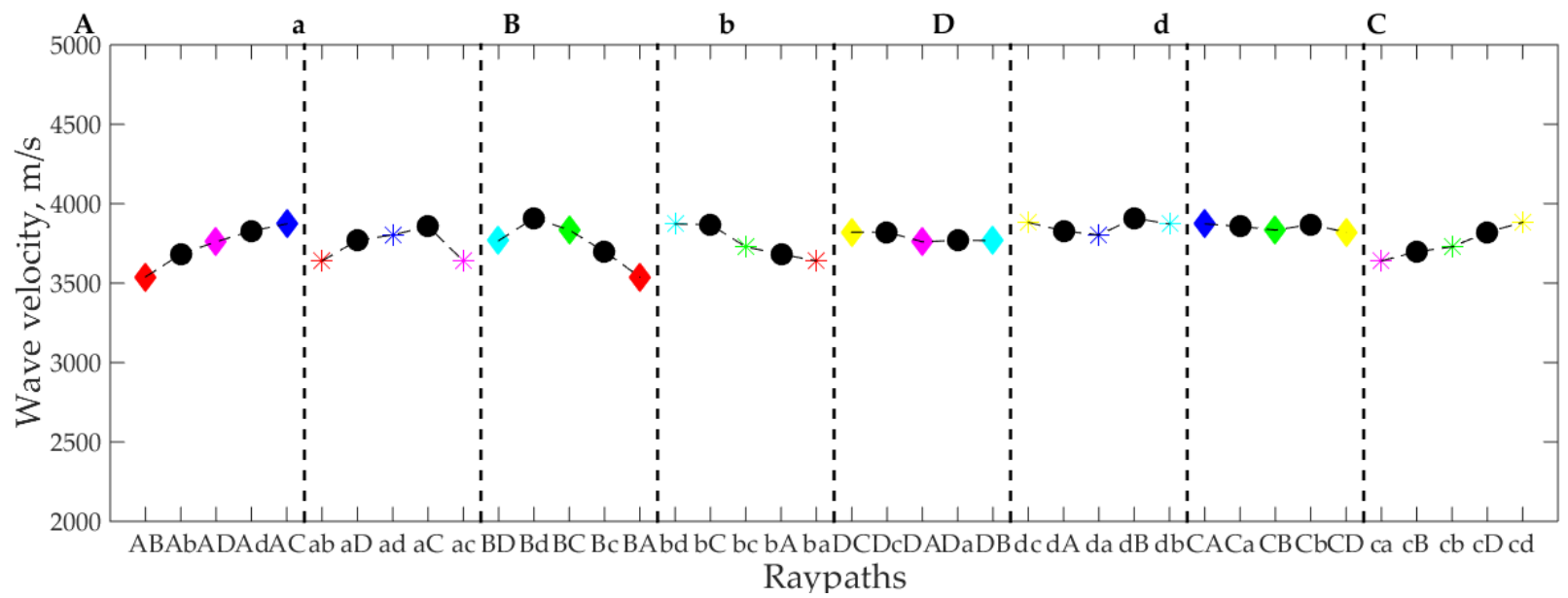

Figure 3.11: Computed velocities for grease only coupling (Roblero, 2017)

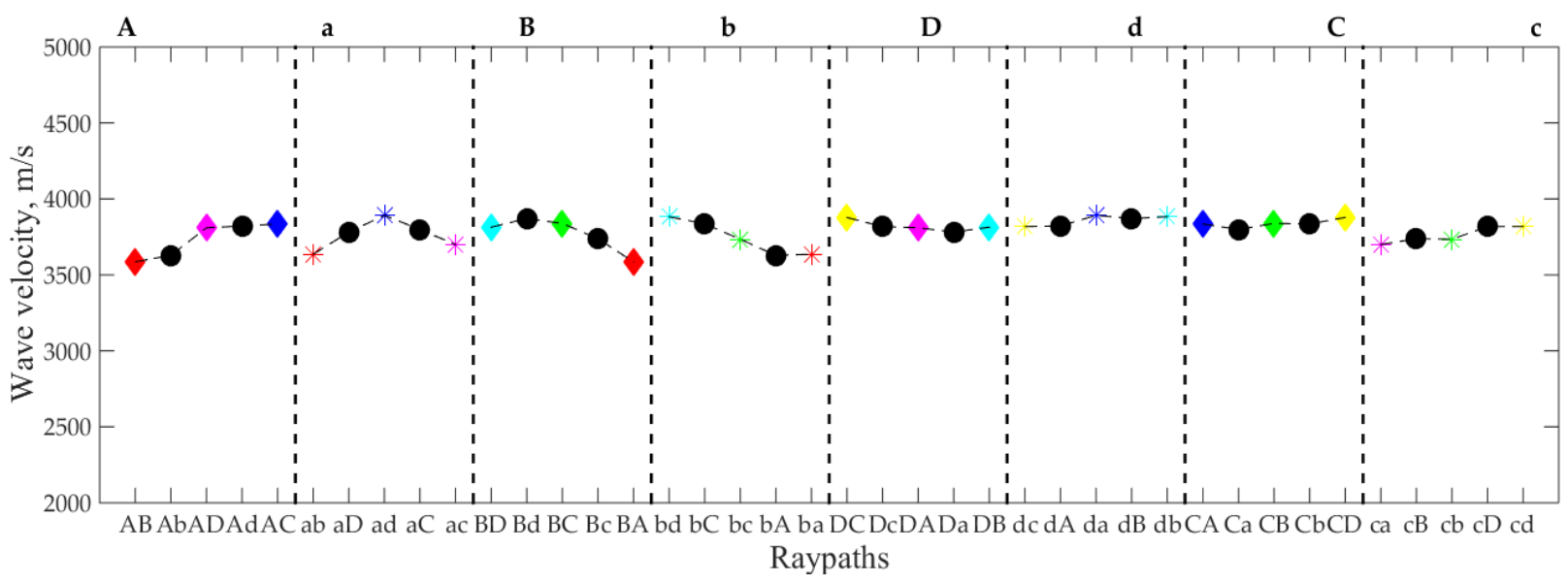

Figure 3.12: Computed velocities for a grease and duct tape coupling system (Roblero, 2017)

The first provides a case for vacuum grease only, while the second provides a case for vacuum grease and duct tape applied. When coupling the systems this way it gives the software two different variables. The goal is to create the best connection between the transducer and the element so that the waves can travel unimpeded. The more the system impedes the wave, the higher the delay will be creating a larger margin of error. By doing the test with multiple coupling setups allows for a wider understanding of the results after completing all of the test runs.

A column that was in-service was determined to be the best situation to get a baseline for the UTPole software in this given example. The in-service column would have been under loads that are experienced through time and fatigue. The assumption would be that this column would 
have been completely sound in strength and would contain no defects. Once the number of paths was decided and the tests were ran, the velocities were calculated (Roblero, 2017). With the new arrival times of the waves in the element the P-wave velocity equation mentioned earlier was used to calculate the velocities. The modulus of elasticity (E) was estimated for the given scenario while the rest were random variables computed by the software. When the testing for the in-service columns came around, a total of 280 were taken on a handful of columns. Each of these columns varied in size, reinforcement, and compressive strength. These variables should change the results and provide different solutions for the test at hand. The reinforcement in the in-service columns also creates a challenge since it is a new parameter for the test and contains different properties when the software runs. When the measurements are ran through the software, the waves should consist of a good correlation to result in a sound and homogeneous element. Good correlation indicates the column has not been compromised or damaged to the understanding of the program. If the results would have shown a negative correlation somewhere, this would be a strong indication of a possible flaw in the element. One ray path that has bad correlation does not necessarily mean that there is damage present, it is just a strong indication that needs another check. From the same study, the two charts below represent the distinction between a sound column and a known column that possesses damage.

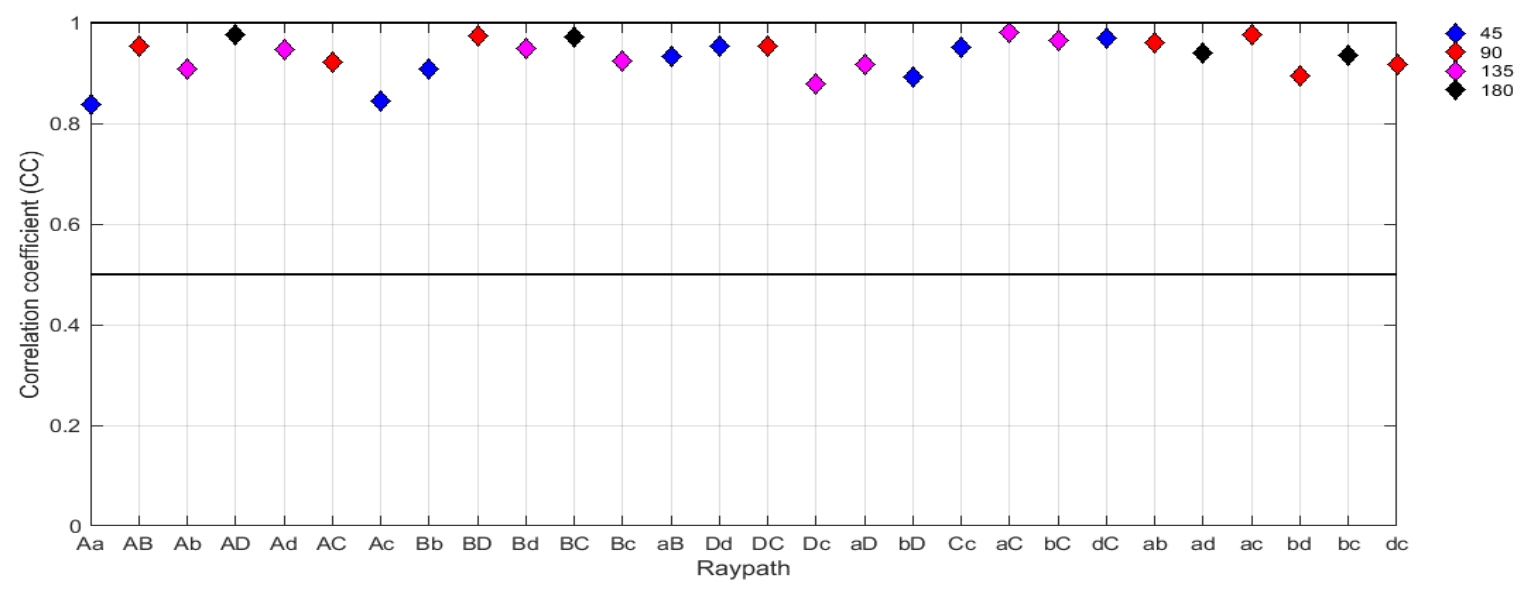

Figure 3.13: Velocities for a sound concrete sample (Roblero, 2017) 


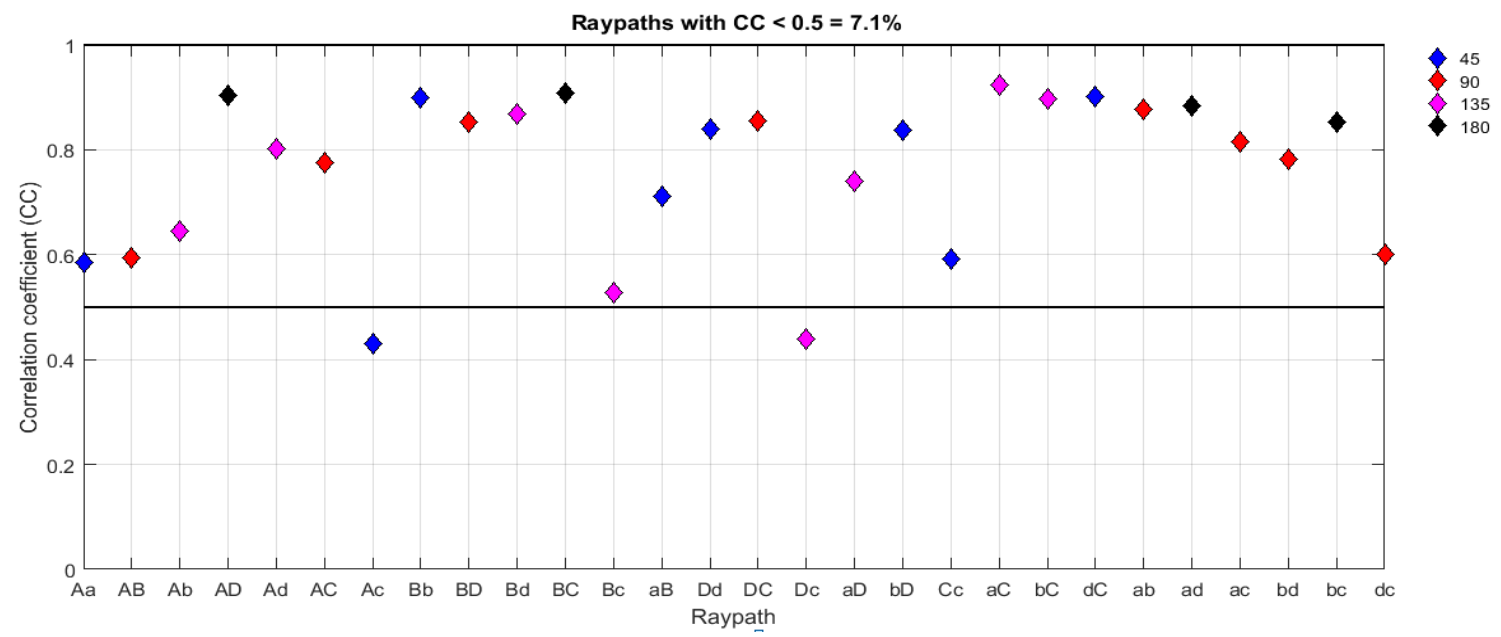

Figure 3.14: Velocities for a concrete sample with known defects (Roblero, 2017)

\section{Conclusion}

When initially beginning the preparation of the test, the device needed to be modified in several way to allow the best setup. The device needed to be able to be portable so that the field operations could be accomplished at an easier rate and the duration of the tests needed to be modified. When multiple tests need to be ran, it is best to keep the run time as short as possible without disturbing the results. A basic engineering background with problem solving skills can be useful even outside of the actual test. Future work will be completed in order to maximize the success of these tests. With the advancement of knowledge in the NDT field, these practices will become more common and lead to a higher demand. Not destroying materials to detect the defects is an extremely important role in cost savings for owners, and will help with overall infrastructure.

\subsection{CONCLUSION}

Ultrasonic testing has become a reliable method to assess various materials and structural members. Ultrasonic techniques provide a fast and efficient way to carry out tests without being too difficult to perform by a trained professional. Ultrasonics in concrete have shown to be a creative solution using new techniques and testing new materials. Ultrasonic velocity tests analysed and compared at different strengths of concrete indicate that each wave reacts differently in the system. Every signal was able to be completed alongside the tests of the uniaxial compression test. Future work would hopefully allow the ultrasonic tests to complete the strength portion of the examination its own. The ultrasonic pulse echo test was able to test a new reactive 
powder concrete at higher levels than the traditional concrete. Future work indicates the higher frequencies will be accomplished using this same technique.

Building new equipment to locate and detect defects in concrete structures is the ongoing process in the ultrasonic industry. With so many large concrete structures to maintain, faster and higher quality work will need to be completed. If the work can be completed in a faster yet more precise manor, the cost for inspection would be capable of dropping tremendously. Early identification of the defects would save billions of dollars in damage repair since the structures would still be in a state where panic and detrimental effects had not started. Limitations still remain in Ultrasonics when testing concrete even with all the success from new ideas and challenges. Limitations in depth penetration and levels of frequencies able to reach in a concrete structure can be enhanced by newer machinery and better setup of equipment. 


\section{APPLICATIONS OF ULTRASONIC TECHNIQUES ON STEEL MATERIALS}

\subsection{INTRODUCTION}

Ultrasonic testing also affects a large portion of infrastructure involving steel members ranging from large structures all the way down to steel welds. The previous chapter discussed some of the applications dealing with steel rebar located in a concrete structure. This chapter will discuss heavily the ultrasonic measurements on smaller pieces in civil infrastructure dealing with connections such as steel welds and joints within a member. Although these sections do not take up as much space as a reinforced concrete structure, they are just as important in keeping the safety of the public. If a joint or weld fails, the entire system also has a chance of a fatal failure since these small areas are holding the systems together. Larger steel beams also deal with ultrasonic testing depending on the situation. Many steel beams are designed and placed in bridges or buildings, and ultimately hold the system in place. Bridge girders made of solely steel contain defects and need inspected on a regular basis.

There are many different types of welds and welding process that take place when constructing. As most challenging trades are, welding takes an extremely experienced and practiced technician to complete the operation. Most welds will still result in having at least a minor defect such as cracks, porosity, slag inclusions, etc. Determining if the defect needs inspected or repaired are typically based off structural codes or standards that are given for the work being completed. For most experienced welders, even with a slight defect passes the standards. Heat-affected zones greatly determine the value and quality in the soundness of the weld since these areas are being heated and cooled multiple times. Metals are also a material to watch when heating and cooling rapidly since the thermal expansion rates are slightly higher than other materials. By controlling the cooling rate of the weld heat affected zone, it allows the weld to better form under the extreme heat. This task can be accomplished by heating the base material prior to performing the actual welding or increasing the heat rate of the system.

Welds still have a high rate of damage if one of the defects takes place in the system. Excessive hydrogen or oxygen when performing a weld can cause gas pockets to form in the metals when the solidification process begins. Even if the air does not contain high amounts of 
common gas that gets trapped in the weld, a high solidification rate (caused by a large difference in temperature between materials) will also cause these gas pockets to form. The table below represents both the causes and remedies for preventing gas pockets or porosity.

Table 4.1.8: Causes and remedies for porosity in welds (Genculu, 2007)

\begin{tabular}{|c|c|}
\hline \multicolumn{2}{|c|}{ Porosity: gas pockets or voids that are found in welds } \\
\hline Causes & Remedies \\
\hline $\begin{array}{l}\text { Excessive hydrogen, nitrogen or } \\
\text { oxygen in welding atmosphere }\end{array}$ & $\begin{array}{l}\text { Use low hydrogen welding process, filler } \\
\text { metals high in deoxidizers, increase shielding } \\
\text { gas flow }\end{array}$ \\
\hline High solidification rate & Use preheat or increase heat input \\
\hline Dirty base metal & Clean joint faces and adjacent surfaces \\
\hline Dirty filler wire & Use clean wire and store fillers in a clean area \\
\hline $\begin{array}{l}\text { Improper arc length, welding current or } \\
\text { electrode manipulation }\end{array}$ & Modify welding parameters and techniques \\
\hline Volatilization of zinc from brass & Use copper-silicon filler, reduce heat input \\
\hline \multicolumn{2}{|c|}{ Porosity: gas pockets or voids that are found in welds } \\
\hline Causes & Remedies \\
\hline Galvanized Steel & $\begin{array}{l}\text { Use E7010 electrode and manipulate the arc } \\
\text { heat to volatilize the galvanizing (zinc) ahead } \\
\text { of the molten weld pool }\end{array}$ \\
\hline $\begin{array}{l}\text { Excessive moisture in electrode } \\
\text { covering or on joint surfaces }\end{array}$ & $\begin{array}{l}\text { Use recommended procedures for baking and } \\
\text { storing electrodes }\end{array}$ \\
\hline High sulfur base metal & Use electrodes with basic slagging reactions \\
\hline
\end{tabular}

Another harmful and common mistake that takes place when welding a member could be caused by incomplete fusion or penetration. If the two metals did not properly fuse together, the weld will be incomplete. Causes of incomplete fusion typically occur when multiple passes are being made without removing the excess slag that in turn covers the weld preventing the fusion from taking place. Lack of penetration also occurs in a weld and forms when the welding rod or joint do not meet the appropriate temperature causing the weld to not go through all the way. If 
penetration does not go all the way through during a weld, the area that normally receives the defect are the sidewalls or at the root of the weld joint.

Any defect in a weld can be harmful to the system, but a crack in the weld leads to catastrophic events in the failure occurs. Hot and cold cracks are both possibilities when performing a welding service. Hot cracks, as they sound, happen when the actual weld is taking place or immediately after before the metal has a chance to cool down. Cold cracks may not take place for a few days, or they could be seen within a few hours at least after the material has returned to a room temperature. There are several causes as to why a weld may crack during or after being applied, the table below demonstrates a few of the causes and remedies for welds cracking.

Table 4.2.9: Causes and Remedies for cracks in welds (Genculu, 2007)

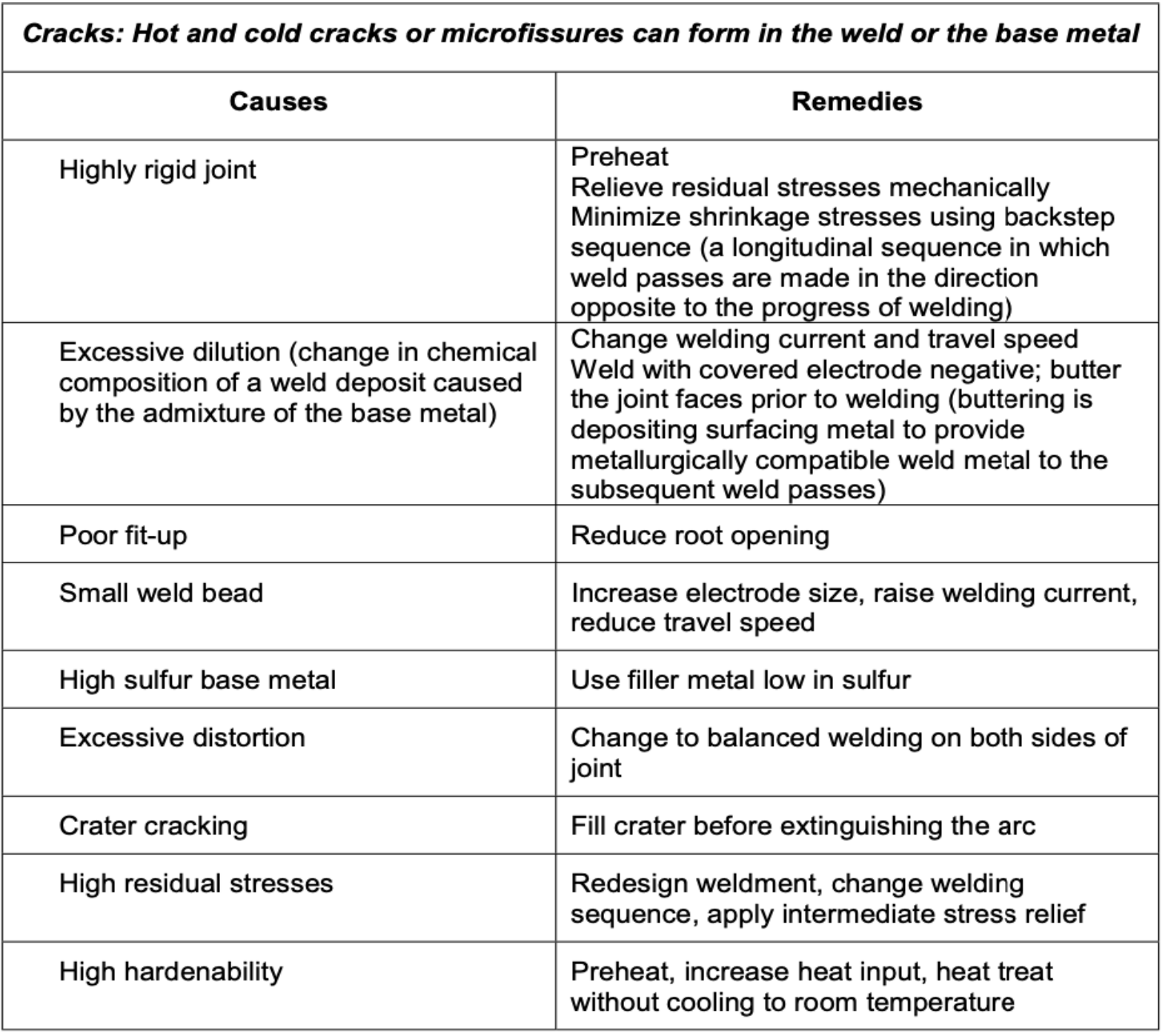


For more information on the types of welds and any certifications for welding, visit the American Welding Society website (https://www.aws.org/home).

\subsection{REVIEW OF APPLICATIONS OF ULTRASONIC TECHNIQUES IN DIFFERENT STEEL MATERIALS}

\subsubsection{A BAYESIAN ESTIMATION-BASED UNCERTAINTY QUANTIFICATION OF FLAWS IN STEEL WELDS DETECTED BY ULTRASOUND PHASED ARRAY (HE ET} AL. 2020)

\section{$\underline{\text { Introduction }}$}

When inspecting for flaws in steel welds and checking the quality of a weld, the phased array ultrasonic scanning method is the most advanced process. The measurements made by the equipment still consist of large uncertainties in the detection results even though the method ranks at the top of the steel weld evaluation. A model by a random field can be generated since steel welds have spatial variability and spatial correlation. The Bayesian uncertainty can be described and processed to allow for the statistical uncertainty to be quantified for the problem. Once the foundation of the Bayesian compressive sensing takes place, the ultrasonic testing for phased array takes place.

\section{Experimental Tests}

The Bayesian compression sensing (BCS) is able to turn an infrequent set of measurements and quantify the statistical uncertainty. Using different vectors and matrices for the given data the BCS converts the information into the statistical uncertainty. Once the probably is found using these principles, the confidence interval can be found for the certain level. When examining a weld, even with the best phased array techniques, the images presented for the weld were inadequate and varied between tests even on the same weld. The measurement data is too sparse for accurate readings. Figure 4.1 demonstrates how two tests on the same weld will vary in results. Information can be perceived and a generalized idea of where defects may be, but the results are different enough to cause the high levels of uncertainty. 


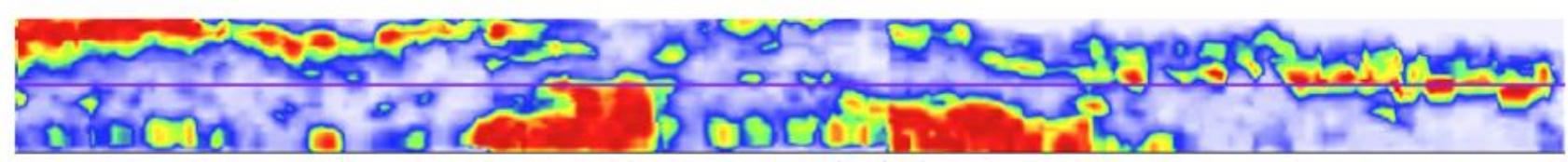

(a) Test \#1 vertical view.

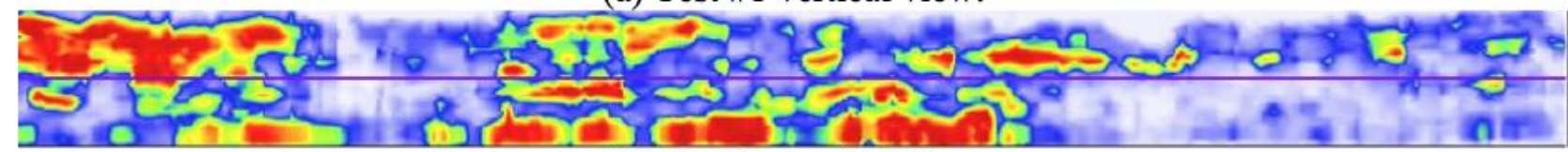

(b) Test \#2 vertical view.

Figure 4.1: Two separate tests conducted on the same weld (He et al. 2020 )

\section{$\underline{\text { Results }}$}

With the BCS completed for the given scenario and the vertical view of the welds, the two methods work together to create a more accurate answer. The two measurements for the steel weld (shown above) are averaged together to create one solid image. Certain peaks that only appeared in one test are eliminated since they may have been out of place or an error on the array results. The weight of the peaks (different color areas) play a role in the BCS to allow for the heavier areas to carry more levels of certainty.

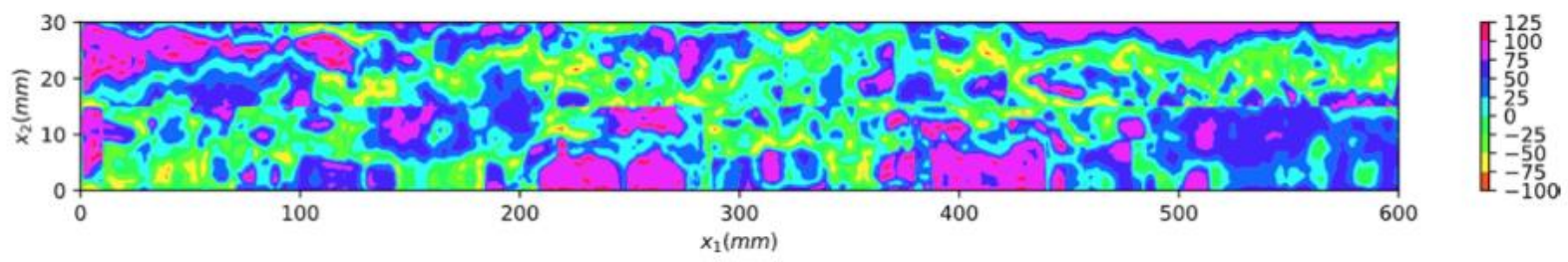

Figure 4.2: Averaged result of the given steel weld (He et al. 2020)

Once the BCS has completed the rest of the certainty on the averaged welds, the confidence interval for the $95 \%$ along the weld are calculated. The flaws for the welds are shown below in figure 4.3 for the $95 \%$ and $99 \%$ confidence intervals in the binomial state fields. 


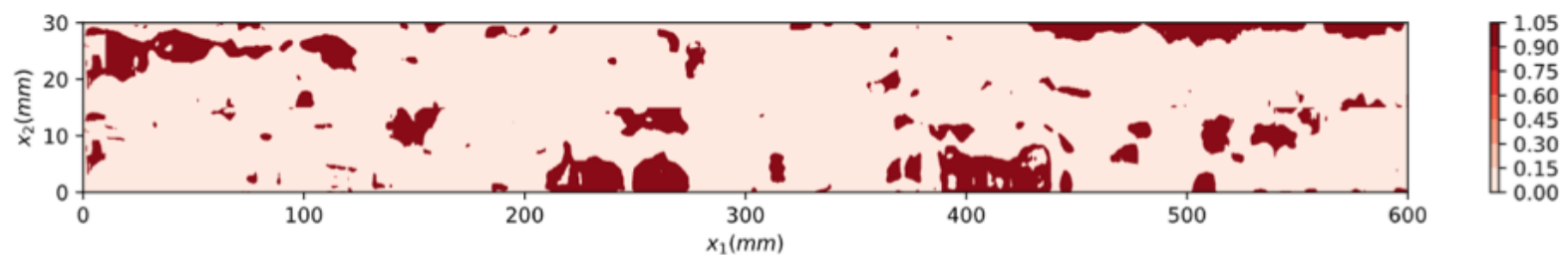

(a) The estimated flaws with $95 \%$ confidence.

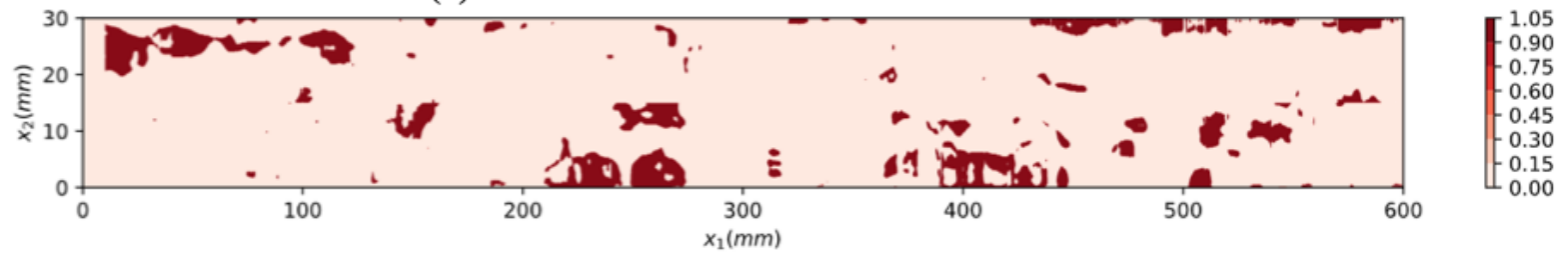

(b) The estimated flaws with $99 \%$ confidence.

Figure 4.3: Flaws for steel welds at $95 \%$ and $99 \%$ confidence intervals (He et al. 2020)

The estimated flaws for the $95 \%$ and $99 \%$ confidence intervals make the uncertainty incredibly smaller compared to the initial results.

\section{Conclusion}

Using the combined methods of the Bayesian compressive sensing and the phased array method of ultrasonic testing creates an accurate measurement for assessing damages or flaws in welds. Since the phased array method finds the flaws in the welds with high levels of uncertainty, the BCS uses the spatial correlation to quantify the statistical uncertainty. Levels of confidence can be calculated for the desired interval using the combined methods.

\subsubsection{ULTRASONIC TESTING AND EVALUATION OF LASER WELDS IN STAINLESS STEEL (GU ET AL. 2013)}

\section{Introduction}

Stainless steel railway car body manufacturing has been using the laser weld technique to reach the higher requirements set forth. The weld produces a more lightweight, higher safety, and better overall appearance for the body of the railway car. Conducting a quality check of the weld at the joints and other key areas prevents the gas pockets from rising to quickly thus causing defects in the weld. The checks all look for areas where the fusion did not take place all the way and the penetration was completed.

Ultrasonic testing methods were examined to try and steer away from the current destructive method of inspecting. The destructive method for inspection of laser welds proves to 
have low efficiency and high costs compared to the alternatives available. Internal characteristics of a laser weld are able to be investigated using a C-scan imaging technique in ultrasonic testing. These characteristics are rapidly discovered for a certain depth in the material and create a structural image based on the scanning. This case study uses $\mathrm{C}$-scan imaging to evaluate the characteristics of the laser weld in stainless steel by modifying the basic echo variation of Ascan.

\section{Experimental Tests}

The experiment for the laser welding process began by using a stainless steel plate (SUS304) with a thickness of $1 \mathrm{~mm}$ and $2 \mathrm{~mm}$ and applying a lap joint weld. The transducer frequency used for the experiment was set at $15 \mathrm{MHz}$ in order to develop a 2D scan of the specimen being examined. Figure 4.4 shows the setup used for the ultrasonic testing of the weld and interface of the base joint. At the interface of the two mediums, the wave reflected and transmitted back to the system. The sound pressure was calculated using the sound of incident wave, sound pression of the reflective wave, and the sound pressure of the transmitted wave. The different acoustic impedance of both mediums also played part in calculating the sound pressure.

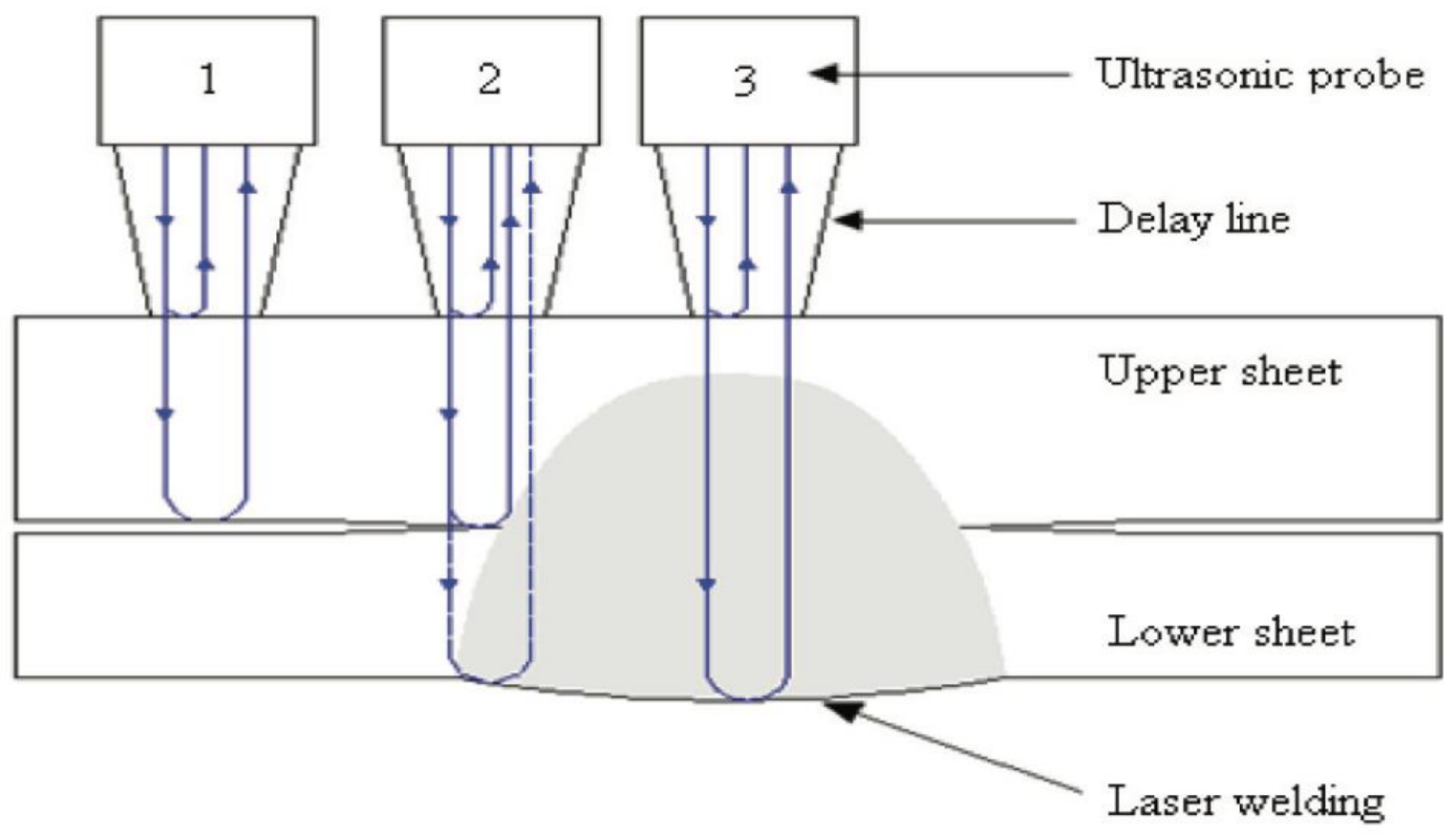

Figure 4.4: Setup for ultrasonic testing method showing the three different locations of testing (Gu et al. 2013) 
When the ultrasonic probe is located at position 3 and sends a wave through the interface of the two materials it indicates the fusion process has been completed and the echo signal covers the entire length of the two mediums. At position 1, there was not a weld connection so the two mediums stay separate meaning the echo does not cross interfaces. Based off the two outside results of the echo signal, there needs to be a weld edge between the two previous results. The weld edge can be defined as the area where a non-weld zone transitions to a weld zone.

\section{$\underline{\text { Results }}$}

The A-scan was found and displayed for the non-weld zone, weld edge zone, and the weld zone between the two mediums. The scan for the non-weld zone showed only a reflected state since the two mediums are separated space. This indicates there are two acoustic impendences with one being air, much lower than that of the stainless steel, so the wave cannot transmit through the material. At the weld edge, the scan reflected at the interface in one area and passed through and reflected at the lower layer in another area. The echo characteristics both areas were represented in the signal for the weld edge since the sound path at the interface and lower region were different. The final zone received the majority of the reflection from the lower level at the bottom of the weld since the fusion of the weld was completed. Since the two materials had fused together, there was still a small amount of reflection from the interface of the weld.

The C-scan imaging showed a plane parallel to the welding surface and vertical to the beam the weld was being applied to by the two plates. Three areas of the weld width were sectioned off and cut to analyze the results of the fusion areas. The first position, $X_{a}$, was located above the area where the two plates were not connected and the metallograph showed the intensity of the first echo did not change in the area meaning the two plates had not been fused together. At the next position, the intensity of the first echo decreases since the position is located above the weld centerline. The weld centerline provided the lowest result for the intensity and gradually increased after moving off the center. The echo characteristics proved to be consistent with the real weld width meaning the weld width is feasible to calculate by using the change of the first echo intensity to the interface of the section.

The analysis using the A-scan and C-scan were completed on 12 stainless steel joints made by laser welding. Seven levels of attenuation were set to calculate the weld width, and the threshold 
of $30 \%$ proved to be provide the highest level of accuracy. Figure 4.5 shows the accuracy from the real measured weld width compared to the results from the $30 \%$ attenuation.

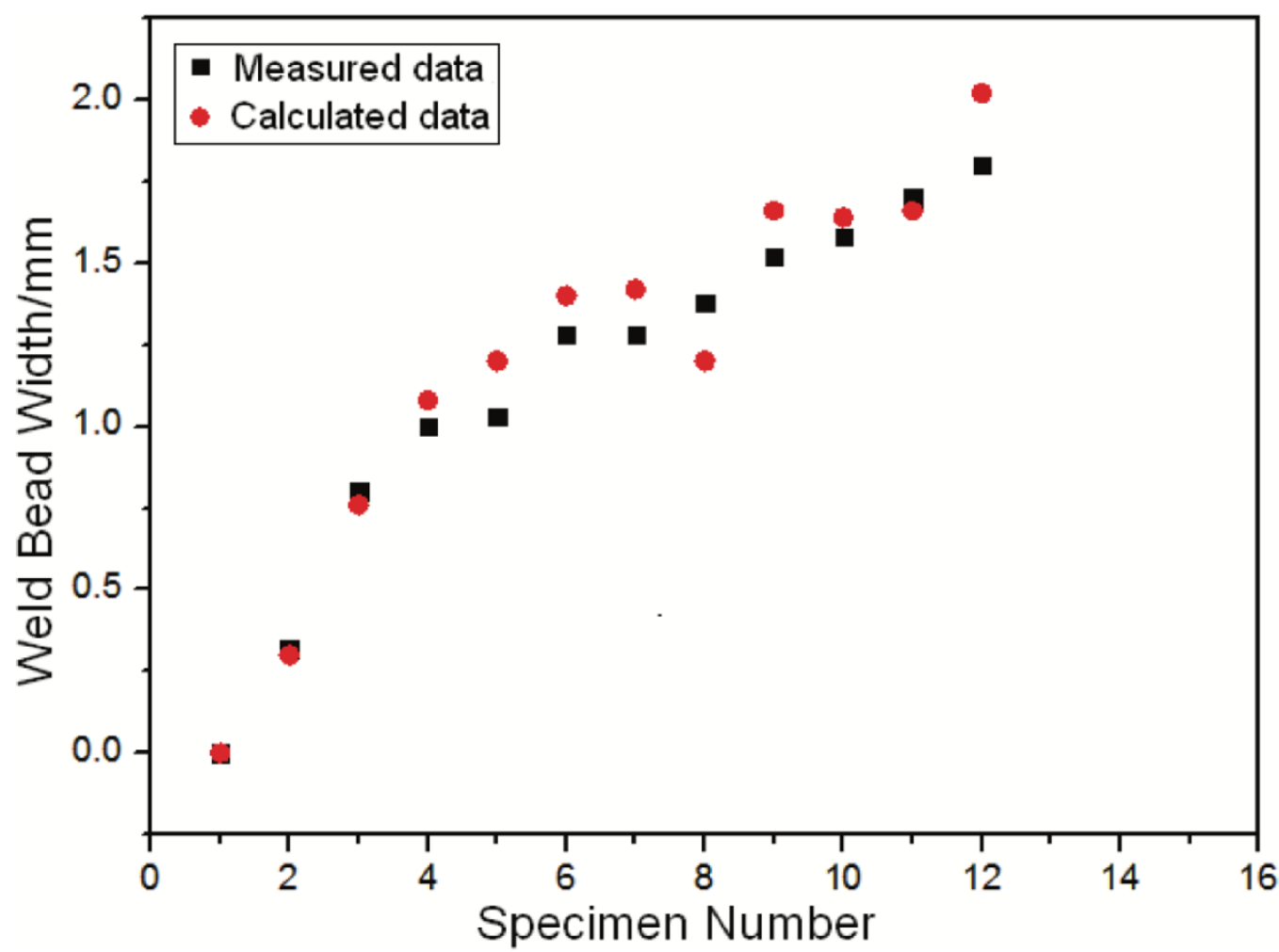

Figure 4.5: Comparison between real measured weld widths and calculate data at 30\% attenuation (Gu et al. 2013)

\section{Conclusion}

The results proved that using a C-scan ultrasonic testing method after an initial A-scan can provide the tester with the condition of the weld and distinguish an area with or without a weld. The experiment also proved that the connection between the first echo intensity and the weld width is validated. Future testing of other materials would need to be conducted to run the same measurements since the properties and characteristics vary from material to material.

\subsubsection{INTERPRETATION OF INDICTATIONS BY SMALL WELDING DISCONTINUITIES IN ULTRASONIC TIME OF FLIGHT DIFFRACTION TECHNIQUE (KACZMAREK ET AL. 2018)}

\section{$\underline{\text { Introduction }}$}

The experiment used time of flight diffraction (TOFD) to examine MAG welded joints and discusses the impact/importance of the technique. Examination for the quality control and 
acceptance of welds remains imperative in the TOFD. Instead of using pulse echo method or radiographic testing methods, TOFD can be used since the equipment is becoming cheaper and more readily available. Complications in the system can be seen when examining the height of a discontinuity found by the TOFD. The record images on the machinery do not correspond to the same dimensions if there are overlapping discontinuities in the sample. The machine prevents the separation of analysis, and becomes more challenging if there is a group of discontinuities.

The case study creates a set of results by examining a member and ultimately sets the stage for future research on the subject line. Research to create an image that represents the same size discontinuities as the ones actually found within the member can be conducted as future work presented by Kaczmarek.

\section{Experimental Tests}

The experimental test relates the nondestructive testing method of TOFD to the regular destructive, metallographic examinations. A MAG weld on the joints was performed for a butt joint with thickness of 15 $\mathrm{mm}$. The experiment setup consisted of an angle beam method where the transducer (SLC563) sat on a wedge set at 70 degrees. The examination was performed using an OmniScan SX flaw detector and semiautomatic HST-Lite scanner. The

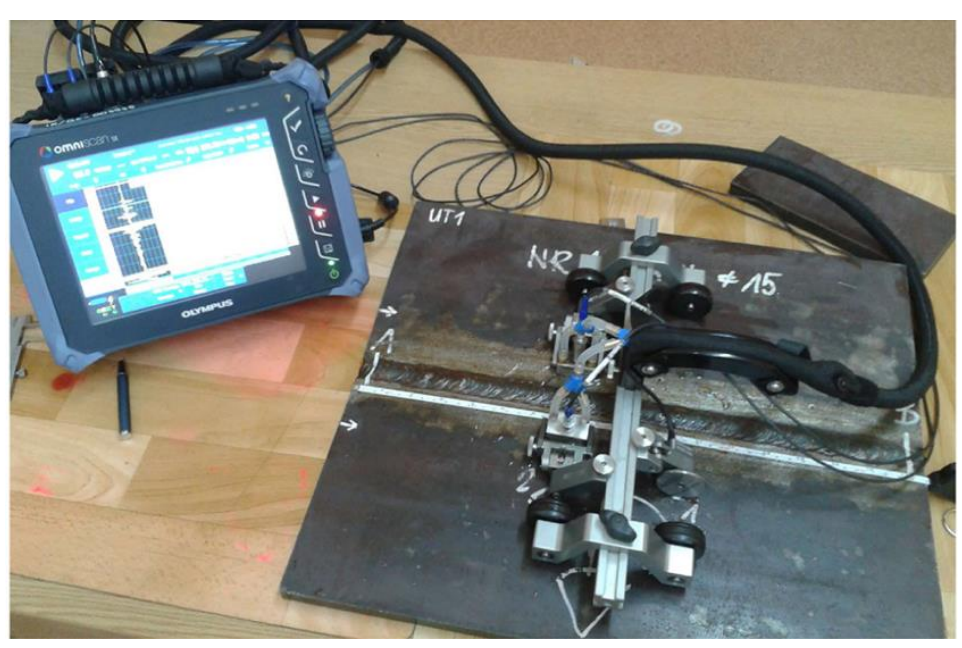

Figure 4.6: Setup for TOFD and equipment used in experiment (Kaczmarek et al. 2018) frequency for the experiment was set at $10 \mathrm{MHz}$ for the probes. The setup and equipment used for the experiment are shown in Figure 4.6 to the right.

\section{$\underline{\text { Results }}$}

The results for the TOFD were verified by the metallographic examination. The TOFD determined the locations of the indications by positioning to the maximum amplitude. The OmniPC computer program recorded the depth and displayed the results on the system. Comparing the results with the metallographic examination, the sample was sectioned through the cross section. By cutting the member, an added error factor for uncertainty was added of plus or minus $1 \mathrm{~mm}$. The metallographic examination confirmed the thought of discontinuities 
located between weld beads at the side of the groove. After comparing all of the data through both methods, the presence of all discontinuities found by the TOFD imaging method were confirmed by the metallographic examination. The figure below indicates the depth of the discontinuities displayed on the device.

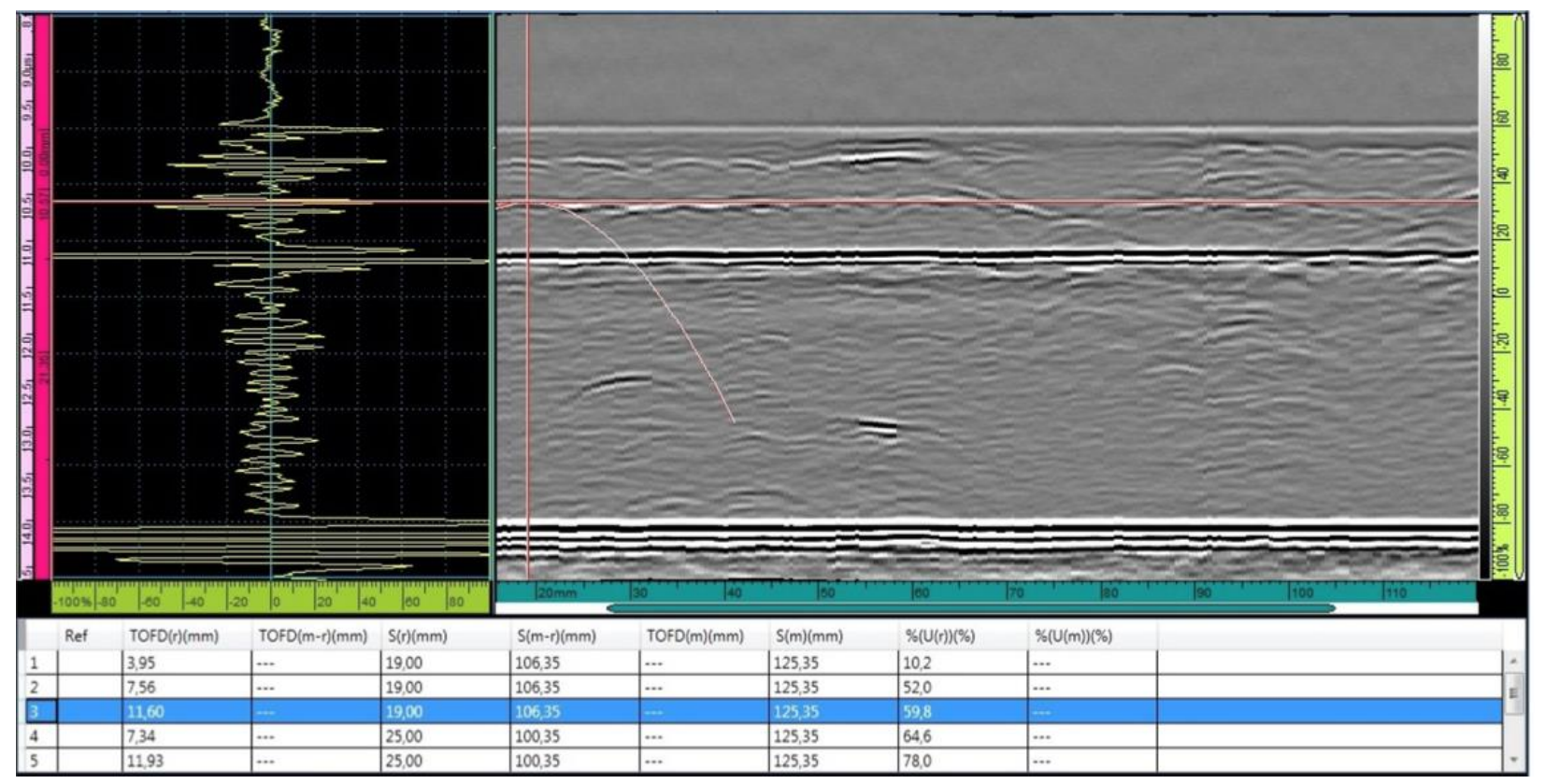

Figure 4.7: OmniPC software displaying the determination and depth of discontinuities (Kaczmarek et al. 2018).

The TOFD was able to indicate the location of the discontinuities, yet unable to measure the height. Indications found at $1 / 4,1 / 2$, and $3 / 4$ of the thickness of the welded joints even though the images presented by the TOFD do not clearly show the measurements since the image is not linear with respect to the depth.

\section{Conclusion}

The time of flight diffraction results confirmed the validity of the methods ability to locate discontinuities in medium. Since the uncertainty of the TOFD alone consisted of high uncertainty in the depths, the metallographic examinations of the MAG welded joints solidified the information presented by the TOFD. The positions and depths of the discontinuities in the medium were confirmed to have heights less the $1 \mathrm{~mm}$ thanks to the metallographic examination. Comparing the results to other methods such as radiographic or other more conventional UT methods, the TOFD and metallographic examination are superior for at least locating a lack of fusion. Other methods are unlikely capable of locating these fusion defects. 
The challenges that surface when interpreting a TOFD image can be seen when there are multiple small discontinuities located in tight position. The metallographic examination has the capability of separating these compact areas and locating the defects. Wave beads in the MAG welds typically generate areas where lack of fusions consist where the two weld beads and the side wall of the groove meet. The table below summarizes the information for the TOFD and the metallographic examination for the different discontinuities. The table demonstrates the depths from both methods and the height from the metallographic test.

Table 4.3.10: Summary table for TOFD and Metallographic Examination for different discontinuities in MAG weld (Kaczmarek et al. 2018)

\begin{tabular}{lllll}
\hline Discontinuity & TOFD & Metallography & & \\
\cline { 3 - 5 } & $\begin{array}{l}\text { Depth of deposition } \\
\text { z }\end{array}$ & $\begin{array}{l}\text { Depth of deposition } \\
\text { center S (mm) }\end{array}$ & $\begin{array}{l}\text { Height of } \\
\text { discontinuity on } \\
\text { section } \mathrm{h}(\mathrm{mm})\end{array}$ & $\begin{array}{l}\text { Depth of deposition } \\
\text { upper edge (mm) }\end{array}$ \\
\hline A1 & 4.0 & 3.47 & 0.34 & 3.3 \\
A2 & 7.6 & 7.71 & 0.43 & 7.5 \\
A3 & 11.6 & 10.68 & 0.16 & 10.6 \\
B1 & 7.3 & 6.53 & 0.27 & 6.4 \\
B2 & 11.9 & 10.48 & 0.57 & 10.2 \\
C1 & 11.7 & 10.49 & 0.78 & 10.1 \\
C2 & 7.6 & 6.48 & 0.17 & 6.4 \\
\hline
\end{tabular}

\subsubsection{A POSSIBILITY OF USING TRANSVERSE ULTRASONIC WAVES TO OBTAIN INFORMATION ON MICROFLAWS IN STEEL (ZHITLUKHINA \&PEROV, 2008)}

\section{Introduction}

Ultrasonic testing methods are capable of indirectly detecting microflaws in the medium of a metal material. The use of longitudinal elastic waves and Raleigh waves with the propagation velocities to find flaws in the medium can be utilized when looking at microflaws since they change the way the wave reacts (Halabe \& Franklin, 2001). Using the Rayleigh waves based ultrasonic methods showed huge advantages compared to other methods, such as the eddy current, because hand held instruments are capable when using the ultrasonic techniques. Trying to compare the ultrasonic methods to the destructive method can prove difficult since the UT methods are usually 1-2\% off for the measurement error on a normal system. When conducting 
the examinations on a microflaw, the amplitude of the elastic waves showed very significant changes. A procedure for detecting the microflaws in the medium based on the parameters to characterize acoustic signals has been proposed. Rayleigh waves were the superior ones chosen for analysis since they are highly sensitive to surface cracks under various conditions. When the member has a painted surface or even rusted areas, the waves showed a much higher attenuation (Franklin \& Halabe, 2014)

An ultrasonic laser interferometer helps provide a contactless method for detecting acoustic fields that help fill that gaps for other conventional ultrasonic methods. The narrow frequency band and low resolution are overcome by visualizing the acoustic field distribution over the specimen due to the receiver at the interferometer. Different degrees of microdamage were obtained by spatial distributions of acoustic noise signals. Field conditions for the test are limited because the equipment is too bulky and expensive to move from the laboratory. Testing at different angle receivers, the tests utilize transverse waves to the ultrasonic laser interferometry.

\section{Experimental Tests}

Different specimen were put under different parameters causing cracks in the 3 metals. A PCUS-10 ultrasonic flaw detector measured the three metals and yielded longitudinal and transverse waves. As microdamage increases in the mediums, the velocity of longitudinal elastic waves decrease as predicted. The metals were formed into $25 \mathrm{~mm}$ in diameter and $5 \mathrm{~mm}$ in thickness disks for testing. A transmitter was on one side of the disk resonating at $5 \mathrm{MHz}$, while the other face was used to detect the acoustic field of longitudinal elastic waves. The results from the ultrasonic beam projection increase with time at a slower rate in a defective medium rather than a defect-free specimen. The microflaws also have a lower propagation velocity than a defect-free system. 


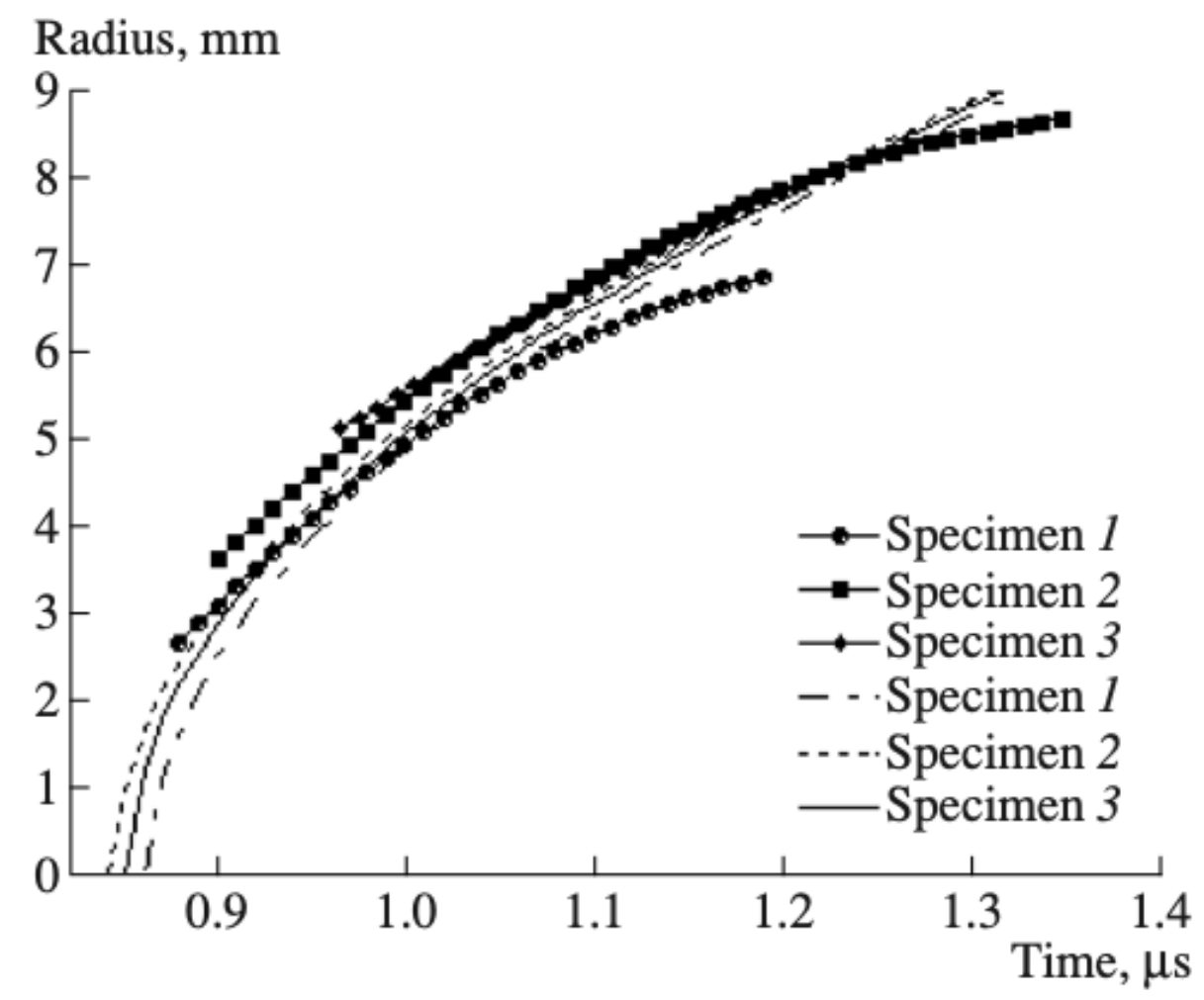

Figure 4.8: Experimental and calculated data for the three specimen with different defect sizes and patterns (Zhitlukhina \& Perov, 2008)

Normal ultrasonic tests have a difficult time understanding and determining the difference in the pulse delay times. Ultrasonic waves are introduced into member at multiple angles when formulating a model. The wave reflects off the bottom of the member and back to the other system on the surface. The wave front propagating in the beam can be assumed to be spherical to make the test simple. Since the propagation velocity in the longitudinal waves is lower the sensitivity for the transverse waves will be higher. When a theoretical model produces results, they are typically 4 to 5 times higher than that of the longitudinal waves.

\section{$\underline{\text { Results }}$}

In the transverse wave direction, the sensitivity increases exponentially as the probe angle increases. After running through multiple calculations using geometry and trigonometry, the simulated data was able to be calculated. An ultrasonic transverse wave beam took a single passage through the specimen thickness for simplicity. A pulsed piezoelectric transducer producer an acoustic field created inside the specimen and on the surface. 

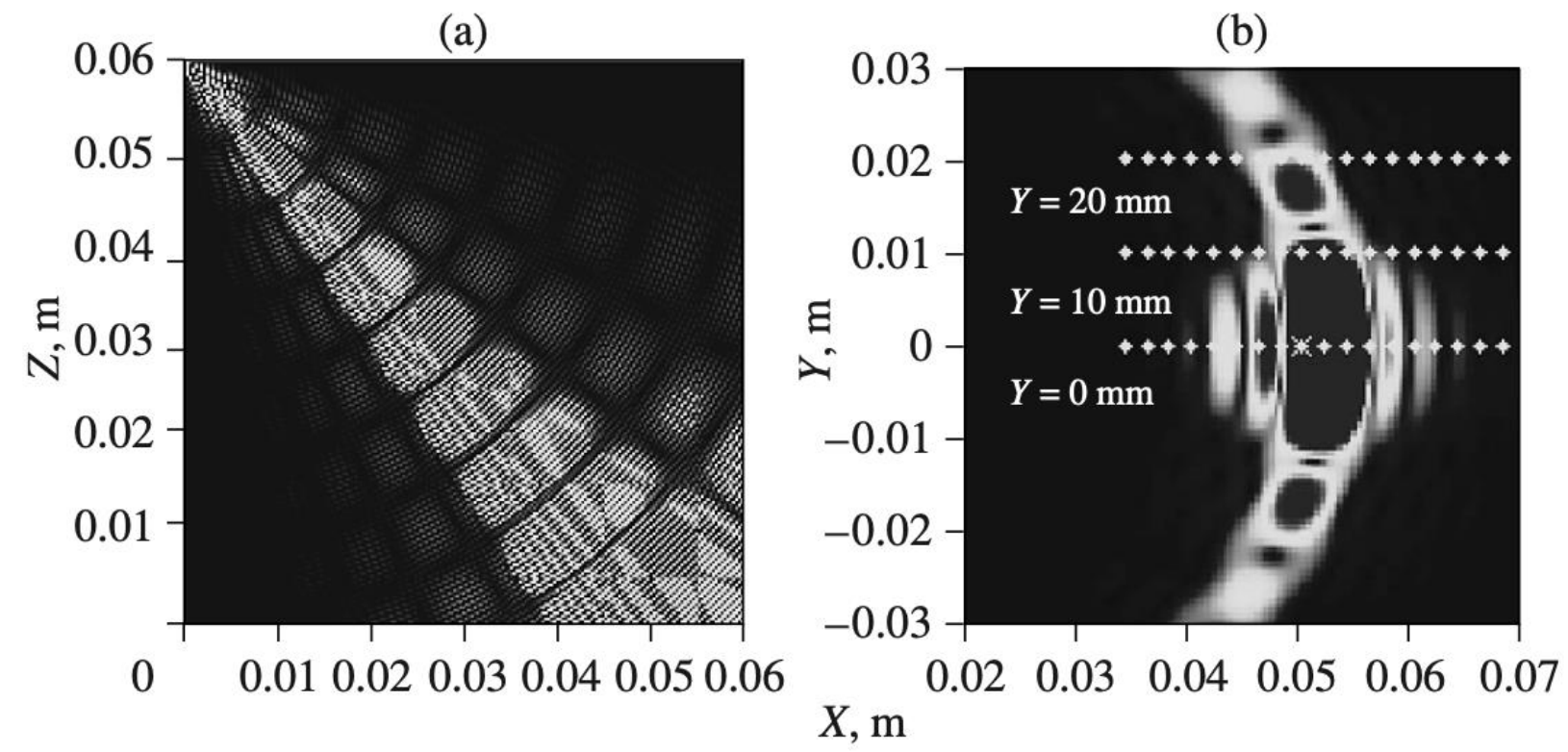

Figure 4.9: Elastic displacements in a trasnverse wave for the (a) inside of the specimen and (b) surface of the specimen (Zhitlukhina \& Perov, 2008).

The sensitivity was estimated for three specimen at including the angle at which the waves were applied. The method has the capability of detecting microdamage in a steam-pipe metal if the specimen is large enough to consider the inner surface of the pipe flat.

\section{Conclusions}

Using the ultrasonic laser-interferometry method, transverse waves and detection of acoustic fields are 2-3 times larger than those of the longitudinal direction when the transducer has an acute angle. The sensitivity achieves the maximum level at 50-60 degrees for the transducer in the simulation. Based on the results, the laser-interferometry method does not produce the most efficient results and other methods may have better turnout.

\subsection{CONCLUSION}

Steel has become one of the key materials in many of the large scale structures that exist in today's infrastructure. Steel members play parts in bridges, buildings, towers on a large scale, but also are key elements holding these structures together with welds and joints. Welds play a large role in the safety and security for these structures that can sometimes be overlooked. When a member in a bridge fails, there are normally safeties in place to ensure that the damage stays in 
a localized area and the damage can be seen well in advance before ultimate failure. If a weld fails with in a bridge or building the chances of having a catastrophic failure is much higher, so the need to detect defects using ultrasonic techniques has become increasingly vital.

Just like concrete having different mixtures and protection, steel members typically have different outside coatings consisting a paint for weather protection all the way to being rusted on the surface. Being able to utilize the correct method and wave pattern for these situations allows for more accurate results. Studies have shown that crack echoes vary for these different coatings, meaning a painted surface reacts in a different manner than rusted mediums (Halabe \& Franklin, 2013).

Advancing the testing equipment and providing easier detection of defects in steel members should be a key concern for the future of NDT. Eliminating expensive testing methods by implementing the ultrasonic tests benefits the economy and the status of civil infrastructure. 


\section{CONDITION ASSESSMENT IN TIMBER STRUCTURES}

\subsection{INTRODUCTION}

Timber has been used as a structural building material for many years since it is from a natural source and has been around for the entire existence of building design. In the modern age, timber can be seen in many different structural elements from building design all the way to wooden poles used for a variety of uses. Timber design, even though most of the wood has been modified to become a stronger structural component, does not have the capability of matching the same strengths and properties compared to other structural elements such as concrete and steel. One of the more common applications for timber can be seen in the use of pile foundations where the surface bearing load does not exceed high standards. These timber pile foundations can be seen historically across the globe and have a high presence in many European countries dating all the way back to the $12^{\text {th }}$ century (Nowak et al. 2019).

Since wood is comprised from a natural material, it tends to biodegrade over time. The strength and properties of the wood depend heavily on the water content and density since it changes quickly if these properties become too extreme. Timber can be defined as a heterogeneous material that unlike others is cellular. From a natural standpoint, timber degrades from a multitude of situations. Shown in Table 5.1 below are the major causes for degradation.

Table 5.1.11: Different types of degradations for timber members (Nowak et al. 2019)

\begin{tabular}{|c|c|}
\hline Type of Degradation & Source of Degradation \\
\hline Biological & $\begin{array}{c}\text { Fungi, bacteria, insects, termites, enzymatic } \\
\text { reactions, oxidation, hydrolysis, reduction, } \\
\text { free radical reactions }\end{array}$ \\
\hline Chemical & $\begin{array}{c}\text { Oxidation, hydrolysis, reduction, free radical } \\
\text { reactions }\end{array}$ \\
\hline
\end{tabular}




\begin{tabular}{|c|c|}
\hline Mechanical & $\begin{array}{c}\text { Chewing dust, wind, hail, snow, sand, stress, } \\
\text { crack, fracture, abrasion, compression }\end{array}$ \\
\hline Thermal & $\begin{array}{c}\text { Lightning, fire, sun } \\
\text { Water }\end{array}$ \\
\hline Water Interactions & $\begin{array}{c}\text { Swelling, shrinking, freezing, cracking, } \\
\text { erosion }\end{array}$ \\
\hline Weather & $\begin{array}{c}\text { Ultraviolet radiation, water, heat } \\
\end{array}$ \\
\hline
\end{tabular}

Flaws can be determined in the timber structural by visual inspection based relatively easy. Typical formations seen on timber elements consist of knots, checks, decay, cracks in different directions, etc. Ultrasonic techniques are capable of analyzing the wood structures in similar patterns to that of concrete or other materials. Wooden structures pass sound waves differently depending on the type of wood used, hardwoods have less resistance to the sound waves allowing them to travel faster compared to softwood. Wooden species can be tested in multiple directions in a cross-section. The annual growth rings grow in a spherical manner where the wave velocities are more efficient and faster in the radial direction compared to the tangential direction. Other angles within the member may be tested and provide separate velocities that are typically lower than the radial and tangential.

Timber materials react differently when exposed to water and the moisture content in the medium. Timber materials tend to soften compared to other materials for a number of reasons with one of them being that it is an organic material. When the medium softens, the modulus of elasticity in the material decreases. Since the wave velocity consists of the square root of the modulus of elasticity (E) divided by the density, the lower the $\mathrm{E}$ value results in the lower wave velocity. This phenomenon can be seen in the case study presented below by Nowak (2019). Timbers with a high moisture rate have much slower wave velocities compared to those with a lower rate consisting of the same material. The modulus of elasticity changes differently between mediums such as timber 
and concrete since the material properties vary. Moisture content in concrete can increase the strength of material rather than decrease it in many cases.

\subsection{REVIEW OF APPLICATIONS OF ULTRASONIC TECHNIQUES IN DIFFERENT TIMBER STRUCTURES}

\subsubsection{ASSESSMENT OF THE CONDITION OF WHARF TIMBER SHEET WALL MATERIAL BY MEANS OF SELECTED NON-DESTRUCTIVE METHODS (NOWAK} ET AL. 2019)

\section{Introduction}

The case study provides methods of determining the condition and parameters for timber structures such as sheet walls or piles that have been submerged in a water-soil environment for service purposes. A timber sheet wall in the Swina strait was examined for the condition because the structure had been in service for nearly 70 years in a low salinity seawater. The history of the sheet provides very little insight because the strait was used for military purposes, but the wall was compiled of tongue and groove jointed timber piles.

To conduct the examination, the sheet wall needed to be removed from the strait and examined comparing the original cross sections to the current (in service) cross sections. The wood experienced different levels of exposure from the top of the structure to the bottom. For the pile to function properly, part of the structure needs to be embedded in a solid material while the other can be exposed, such as a vertical cantilever. As shown in Figure 5.1 below, part of the pile was embedded into the soil while the other half resided in the low salinized water. The very top of the pile also experiences an affect caused by the waves impacting the structure (related to a fatigue effect since the waves are constantly impacting the structure in a cyclic manor). 


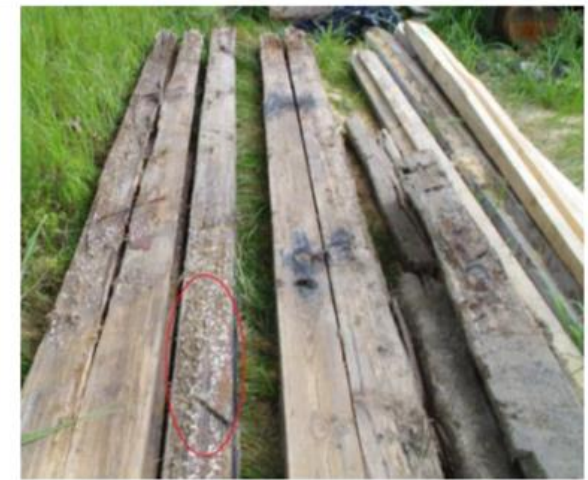

(a)

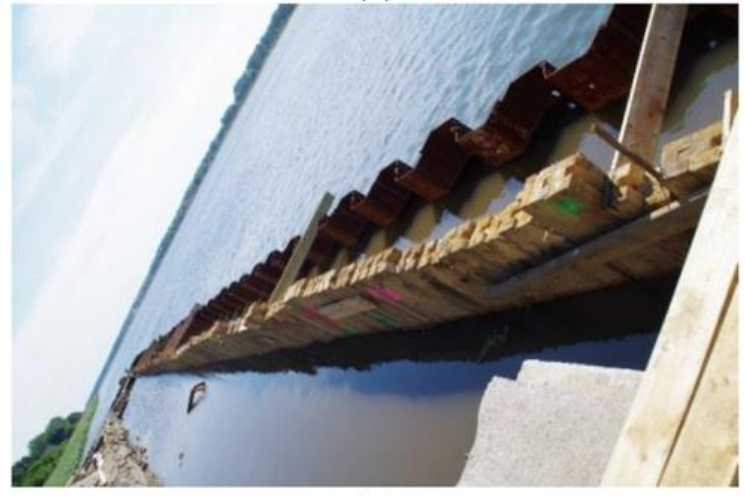

(c)

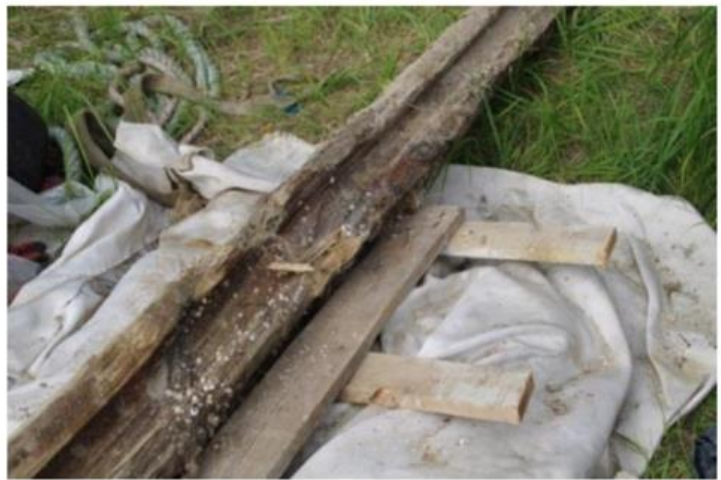

(b)

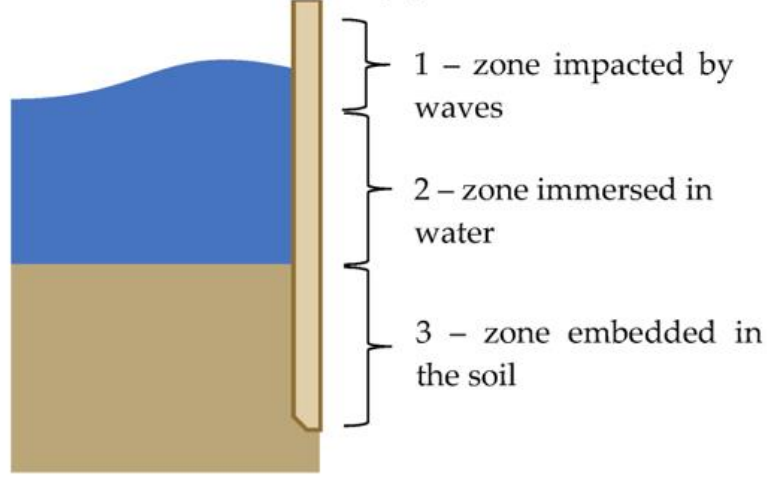

(d)

Figure 5.1: Detailed view of piles dismantled and the different zones where the pile was affected (Nowak et al. 2019) The areas exposed to the waves in the piles had the highest degree of change and structural damage since it experienced the worst load. The area highlighted with the red circle in Figure 5.1 shows the top part at the pile exposed to these waves.

The objective for the study was to assess the condition of the in-service wooden members using NDT and to develop a methodology for testing the sheet wall. The report shows the quality of wood subjected to various environmental conditions and how the structural integrity changes based on the different conditions.

\section{Experimental Tests}

Using ultrasonic testing methods on timber analysis instead of destructive methods provides the user to examine the structure while keeping it fully intact. By analyzing the velocity of wave propagation induced in a member, the mechanical properties and internal discontinuities can be determined. The destructive methods are also not capable of detecting the internal damage or defects in the wood. The wave traveling through the member can be measured between two points, covered by the excited state by the wave, over a period of time produces the velocity in 
the member. When inspecting the different species of wood, the grain size and direction either along or across the grain. The size along the grain ranges from 3-5 times larger than across the grain. For a sound wave sent through the grains the velocity for the wave propagation can amount to a range of $3500-5000 \mathrm{~m} / \mathrm{s}$ for the P-wave and only amount to $1000-1500 \mathrm{~m} / \mathrm{s}$ for the Swave (Feio et al. 2007). The configuration of the natural wooded material provides more cell walls and interferences in the transverse direction slowing the sound wave velocity compared to the longitudinal direction where fewer obstacles are present.

The equipment used in the study to produce the ultrasonic waves can be seen in the figure below. The Fakopp Microsecond Timer was the device used for complete NDT, while the Sylvatest Trio needed to drill $5 \mathrm{~mm}$ wide and $10 \mathrm{~mm}$ deep holes into the specimen being tested for the probes. Both devices send an ultrasonic wave into the medium to secure the results. The Fakopp Microsecond Timer uses two probes on each side of the sample to conduct the test and a single strike from a special hammer excites the waves in the material. Fakopp produces multiple other NDT machines to provide other modes of inspection. Different point and directions were tested to better determine accurate results for the medium. 


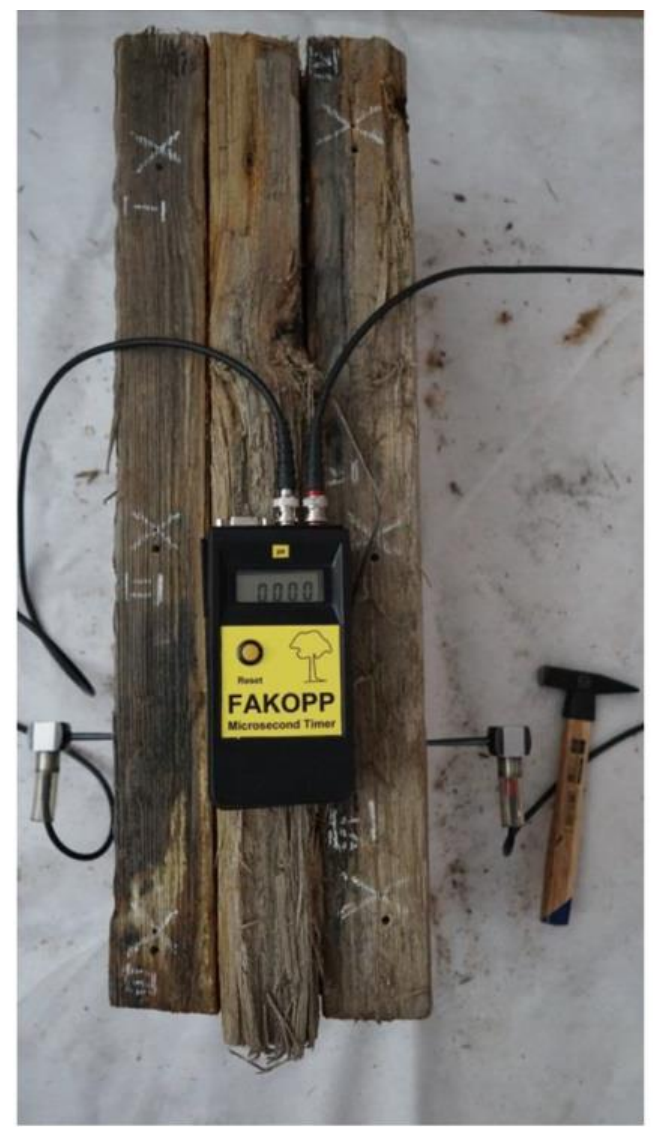

(a)

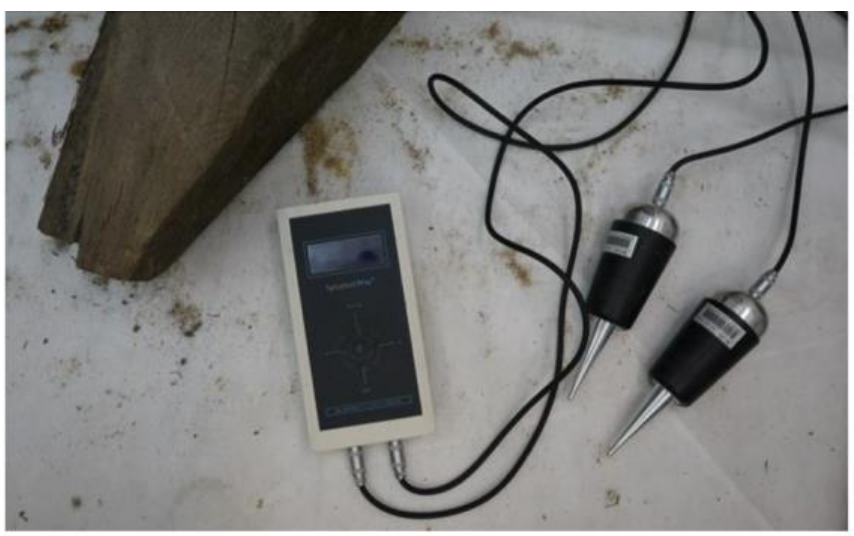

(b)

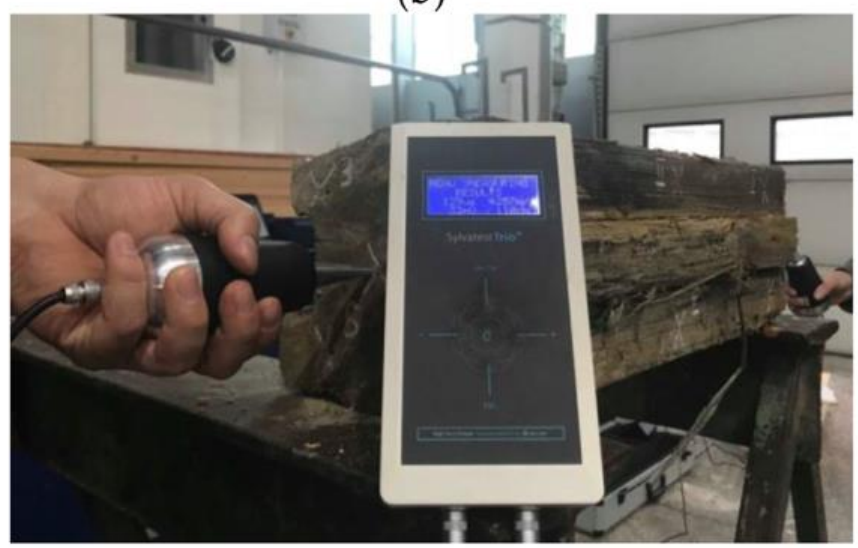

(c)

Figure 5.2: Example setup for (a) the Fakopp Microsecond Timer and (b) Sylvatest Trio Device (Nowak et al. 2019)

To conduct the test, an $18 \mathrm{~mm}$ x $20 \mathrm{~mm}$ x $60 \mathrm{~cm}$ sample cuts were taken from the removed timber wall. Samples were pulled from each of the zones previously mentioned since the structural integrity and properties would be different because of exposure to the different scenarios. The type of material the wharf timber sheet wall was made of pinewood. Other species of wood could drastically change the results. Once the equipment ran the tests for the stress waves, the destructive test was conducted by drilling into the medium. 


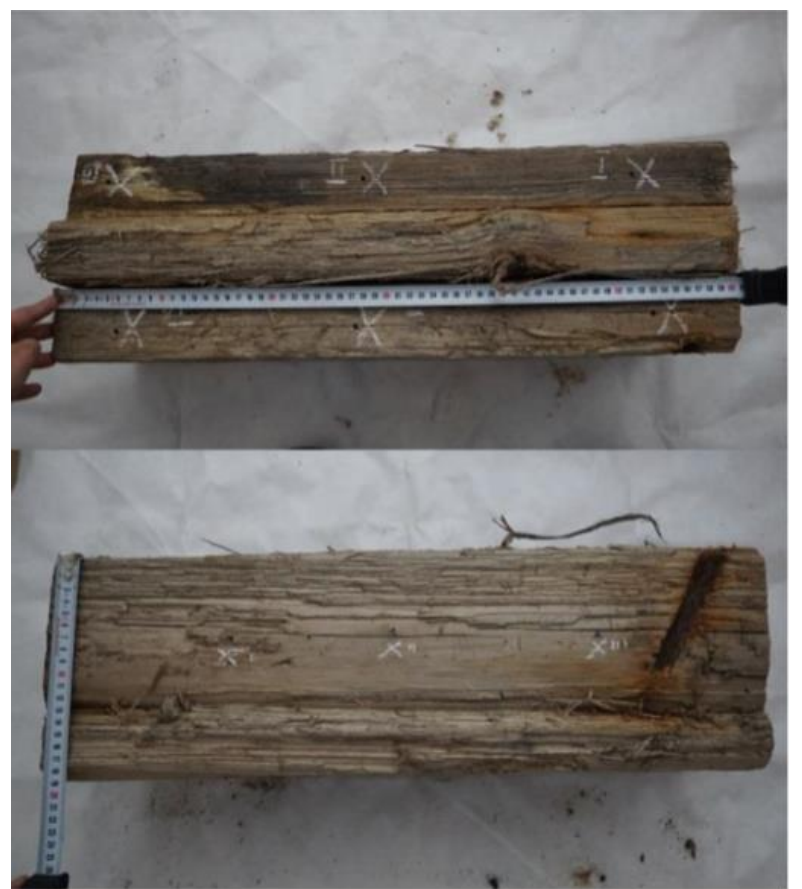

(a)

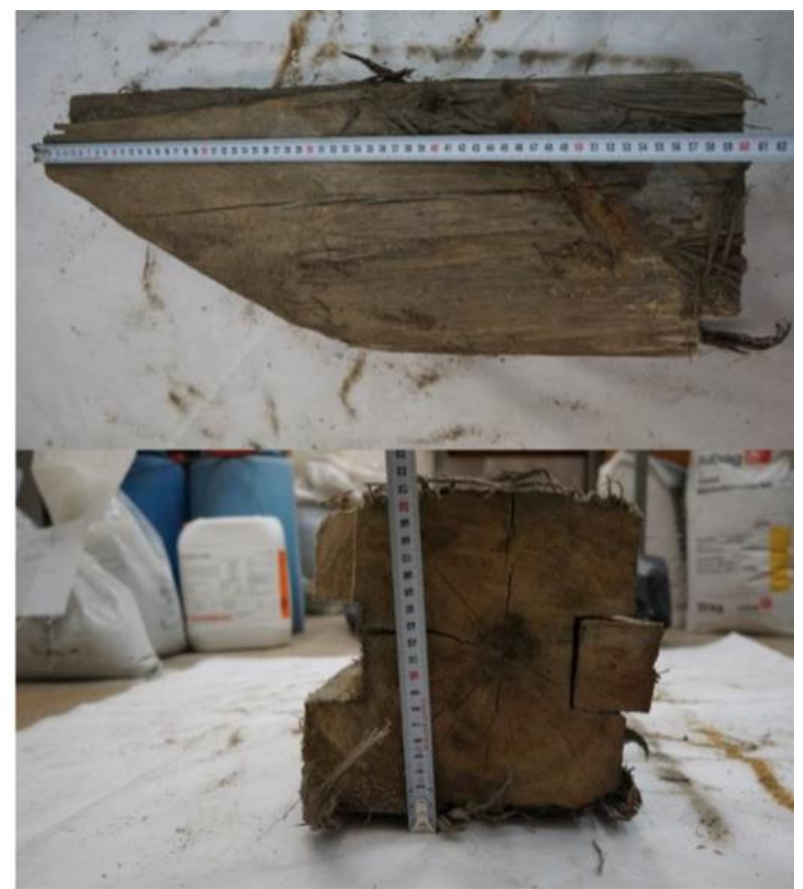

(b)

Figure 5.3: Shown are (a) sample taken for test. (b) Sample for tests with a cut (Nowak et al. 2019)

\section{$\underline{\text { Results }}$}

The densities were measured and recorded for the 40 samples that contained no flaws so data could be used later for the ultrasonic testing along with the moisture content by a resistancetype moisture meter.

The velocity for the wave propagation in the medium were calculated using the described methods above. Using the velocities and the calculated densities mentioned above, the dynamic moduli of elasticity was also calculated for the medium. The velocities of the stress waves and the dynamic modulus of elasticity for both the Fakopp Microsecond Timer and the Sylvatest Trio are shown in the tables below for both the parallel and perpendicular directions of the grain.

Both of the methods can be considered complimentarily to estimate the material parameters of wood. 
Table 5.2.12: Fakopp Microsecond Timer Results for the velocities and dynamic modulus of elasticity at different moisture contents (Nowak et al. 2019)

\begin{tabular}{|c|c|c|c|c|c|c|c|}
\hline \multirow{3}{*}{ Sample } & \multirow{3}{*}{$\begin{array}{c}\text { Direction Relative to } \\
\text { Grain }\end{array}$} & \multicolumn{3}{|c|}{$\mathrm{V}[\mathrm{m} / \mathrm{s}]$} & \multicolumn{3}{|c|}{$\mathrm{MOE}_{\text {dyn }}[\mathrm{GPa}]$} \\
\hline & & \multicolumn{3}{|c|}{ Moisture Content } & \multicolumn{3}{|c|}{ Moisture Content } \\
\hline & & $\sim 30 \%$ & $\begin{array}{l}24- \\
28 \%\end{array}$ & $\sim 18 \%$ & $\sim 30 \%$ & $\begin{array}{l}24- \\
28 \%\end{array}$ & $\sim 18 \%$ \\
\hline \multirow{2}{*}{1} & parallel & 4872.8 & 5376.6 & 5644.8 & 11.22 & 13.66 & 15.06 \\
\hline & perpendicular & 1153.7 & 1439.9 & 1443.8 & 0.63 & 0.98 & 0.98 \\
\hline \multirow{2}{*}{2} & parallel & - & - & - & - & - & - \\
\hline & perpendicular & 1218.5 & 1372.8 & 1270.6 & 0.71 & 0.90 & 0.77 \\
\hline \multirow{2}{*}{3} & parallel & - & - & - & - & - & - \\
\hline & perpendicular & 1406.1 & 1447.7 & 1665.2 & 1.14 & 1.21 & 1.60 \\
\hline
\end{tabular}

Table 5.3.13: Sylvatest Trio results for the velocities and dynamic modulus of elasticity at different moisture contents (Nowak et al. 2019 )

\begin{tabular}{|c|c|c|c|c|c|c|c|}
\hline \multirow{3}{*}{ Sample } & \multirow{3}{*}{$\begin{array}{c}\text { Direction Relative to } \\
\text { Grain }\end{array}$} & \multicolumn{3}{|c|}{$\mathrm{V}[\mathrm{m} / \mathrm{s}]$} & \multicolumn{3}{|c|}{ MOE $_{\text {dyn }}$ [GPa] } \\
\hline & & \multicolumn{3}{|c|}{ Moisture Content } & \multicolumn{3}{|c|}{ Moisture Content } \\
\hline & & $\sim 30 \%$ & $\begin{array}{l}24- \\
28 \%\end{array}$ & $\sim 18 \%$ & $\sim 30 \%$ & $\begin{array}{l}24- \\
28 \%\end{array}$ & $\sim 18 \%$ \\
\hline \multirow{2}{*}{1} & parallel & 5128.9 & 5855.4 & 6035.2 & 12.43 & 16.20 & 17.21 \\
\hline & perpendicular & 1118.4 & 1311.5 & 1356.9 & 0.59 & 0.81 & 1.04 \\
\hline \multirow{2}{*}{2} & parallel & - & - & - & - & - & - \\
\hline & perpendicular & 1030.2 & 1311.4 & 1481.1 & 0.50 & 0.82 & 1.04 \\
\hline & parallel & - & - & - & - & - & - \\
\hline & perpendicular & 1199.8 & 1251.1 & 1422.3 & 0.68 & 0.90 & 1.04 \\
\hline
\end{tabular}




\section{Conclusion}

The case study proves that using NDT methods such as ultrasonic techniques provide a reliable assessment of the technical condition of timber. Even with the recommendation of using these methods, the tests should also be conducted using the visual technique since it can be the first sign of needing tested. Since there are not any comprehensive studies for this area of technique, the information for the parameters and destruction are limited. Currently there are not any methods of ultrasonic testing or NDT that have the capability of testing the condition of timber for the strength characteristics of wood. The methods have the capability of detecting defects such as the knots in the wood, but not the strength parameters.

The two methods of acoustic testing prove to be an efficient way to test for the velocities and the tests seem reliable. As the moisture content in the wood increases, the wave propagation velocity decreases. Further research is required for the subject to better establish ways to estimate the material stiffness parameters for wood structures. Mechanical parameters are capable of being recorded based on the determined modulus of elasticity. The methods of ultrasonic testing have the capacity to calculate the local state of the material, but require other methods to help provide the global state of the material for in situ testing which explains the reason for the other testing's.

\subsubsection{SPACING FOR ACCURACY IN ULTRASONIC TESTING OF BRIDGE TIMBER PILES (MCUEN ET AL. 1988)}

\section{Introduction}

Timber bridge piles have been a part of American civil infrastructure for over a century. Today's standards at the federal and state levels call for periodic inspection of all bridges across the country including the safe load carrying capacity. Old historical bridges have become unstable with timber piles because the natural wood contains properties that are susceptible to deterioration. The inspection for these timber bridges allows them to be rebuilt or repaired when needed. If the inspection never takes place or a poor job of inspection happens, the chance of failure in the bridge has increased odds.

Visual inspection, the standard practice for conducting timber bridges, cannot determine the material strength. An example of this case, presented by McCuen, was found in Maryland when 
an underwater inspection in 1975 of bridge piles was inspected visually. The bridge passed inspection, but ultimately failed roughly a year after. The soundness of the material passed for the visual inspection, but laboratory testing proved the material strength decreased over the life of the pile. Bridges made from timber experience the same impact, fatigue, overloading situations that standard bridges face, but also experience the more biological decays such as decay fungi and insect attack.

The goal of the study was to determine the appropriate spacing for testing of any material with specific accuracy of results for engineers in the field.

\section{Experimental Tests}

Timber bridge piles appear above and below water for tests that involve using ultrasonic testing techniques. The ultrasonic techniques pair with compressive tests to determine strength and material properties for the members. The pulses for the test were sent in the radial direction of the timber piles for a direct transmission setup. The strength for the cross-section varies, so the setup shown below provides the best results for an in service strength test. The direct transmission method was preferred for the timber bridge pile analysis for a couple reasons, with one of them being the path length was well defined since the transducers were just on opposite sides. 


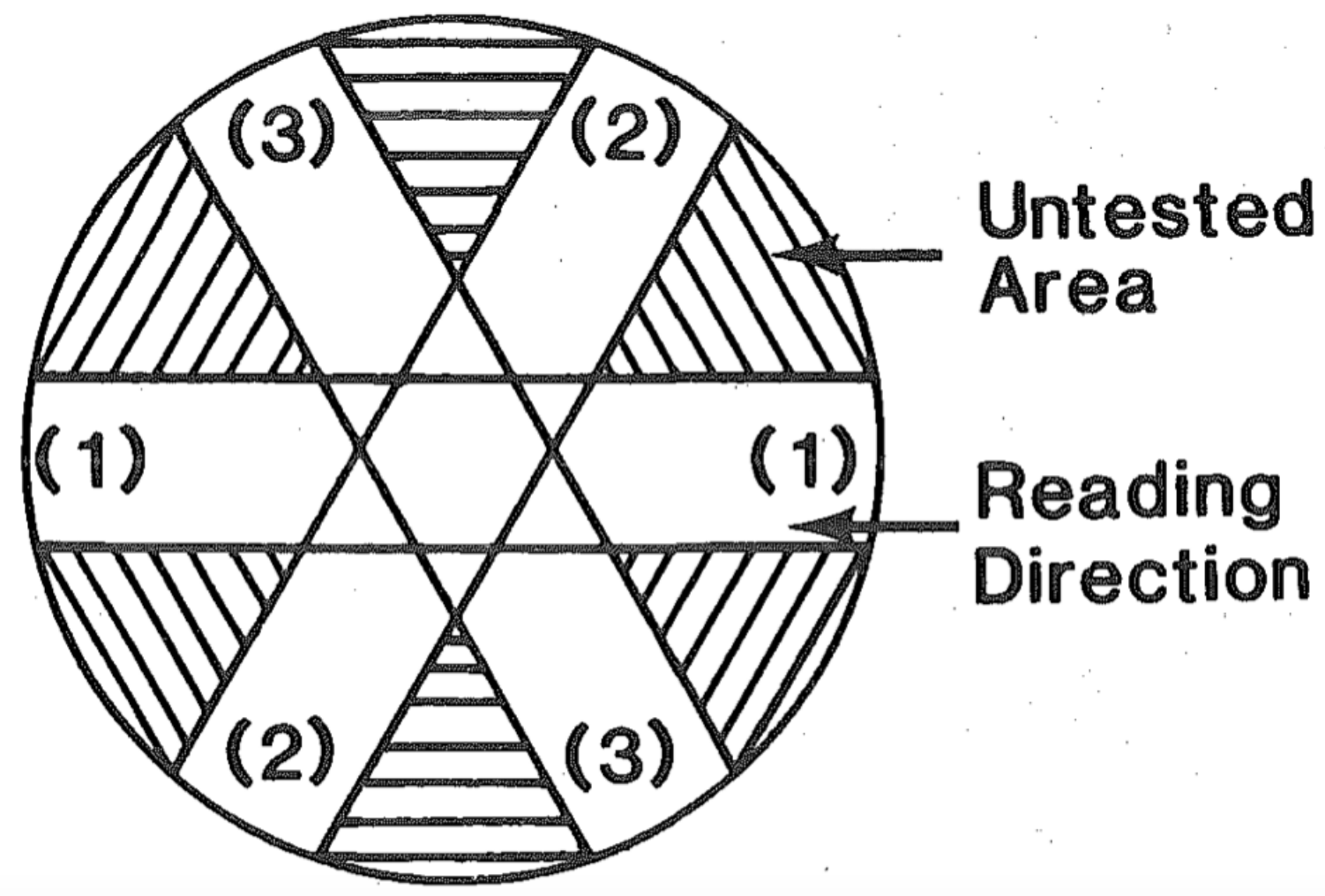

Figure 5.4: Cross-section of timber bridge pile showing setup for the ultrasonic testing (McCuen et al. 1988)

The test consisted on two $54 \mathrm{kHz}$ transducers, one for transmission and one for receiving, placed on each side of the pile. The test was completed for above and below water sections on the piles meaning the equipment had to be firmly secured. Because of the natural properties of timber, factors such as moisture content, treatment, type of wood had to be considered. Assumptions about the structure must be made to develop spacing requirement guidelines. The properties must be viewed as random variables because the properties change on a microscopic level. A onedimensional variable was the only variable being considered since the size in the length of the member is the only stochastic characteristic of interest. A semi variogram analysis and kriging estimation are the methods used to develop the guidelines for non-destructive testing for timber piles.

The test results for a different paper were completed on old piles that were in service and new piles that were purchased so the initial results could be completed. Both piles, old and new, were yellow-pine sections. The case study McCuen put together had several piles from 4 separate 
bridges. 9 piles were tested for their wave velocities along the length in either one, two, or three directions. The breakdown of the pile combinations are shown in the table below along with the semi variogram parameters included.

Table 5.4.14: Bridge piles with direction analysis was completed (McCuen, 1988)

\begin{tabular}{|c|c|c|c|}
\hline \multirow[b]{2}{*}{$\begin{array}{l}\text { Bridge } \\
\text { (1) }\end{array}$} & \multirow[b]{2}{*}{$\begin{array}{l}\text { Pile/direction } \\
\text { (2) }\end{array}$} & \multicolumn{2}{|c|}{ Estimate of } \\
\hline & & $\begin{array}{c}\text { Sill }(\mathrm{ft} / \mathrm{sec})^{2} \\
(3)\end{array}$ & $\begin{array}{c}\text { Range of influence }(\mathrm{ft}) \\
(4)\end{array}$ \\
\hline \multirow[t]{2}{*}{ Denton } & $\mathrm{DB} / 1$ & 51,000 & 1.5 \\
\hline & $\mathrm{DB} / 2$ & 91,000 & 1.5 \\
\hline \multirow[t]{3}{*}{ Denton } & $\mathrm{DD} / 1$ & 56,000 & 1.0 \\
\hline & $\mathrm{DD} / 2$ & 83,000 & 1.0 \\
\hline & $\mathrm{DD} / 3$ & 122,000 & 1.0 \\
\hline \multirow[t]{3}{*}{ Sandyfield } & $\mathrm{SB} / 1$ & 380,000 & 1.5 \\
\hline & $\mathrm{SB} / 2$ & 160,000 & 1.5 \\
\hline & $\mathrm{SB} / 3$ & 249,000 & 1.5 \\
\hline \multirow[t]{2}{*}{ Marshyhope } & $\mathrm{MG} / 1$ & 650,000 & 4.5 \\
\hline & $\mathrm{MG} / 2$ & 700,000 & 4.5 \\
\hline \multirow[t]{2}{*}{ Marshyhope } & $\mathrm{M} 2 / 1$ & 500,000 & 9.0 \\
\hline & $\mathrm{M} 2 / 2$ & 970,000 & 7.5 \\
\hline \multirow[t]{2}{*}{ Marshyhope } & M3/1 & $1,100,000$ & 7.5 \\
\hline & $\mathrm{M} 3 / 2$ & 970,000 & 7.0 \\
\hline \multirow[t]{2}{*}{ Smithville } & $\mathrm{UA} / 1$ & 860,000 & 3.0 \\
\hline & $\mathrm{UA} / 2$ & 720,000 & 2.0 \\
\hline \multirow[t]{2}{*}{ Smithville } & $\mathrm{UB} / 1$ & 180,000 . & 2.0 \\
\hline & $\mathrm{UB} / 2$ & 780,000 & 3.0 \\
\hline Smithville & $\mathrm{UC} / 2$ & 460,000 & 3.5 \\
\hline
\end{tabular}

\section{$\underline{\text { Results }}$}

The minimum point strength was found for the piles and then converted into a velocity for the mean value at a sampling point. The sampling error or first source of variation was expected to be low because the $\mathrm{R}^{2}$ values were $97 \%$ and $98 \%$. The second element in the sampling program was used to predict the number and spacing points for testing. The sampling points depend on the material that the test works on. A homogeneous pile would have the optimum sampling plan 
provide minimum expected error. For a nonhomogeneous pile, have a higher variance of the measurements, meaning decay is more likely to be detected.

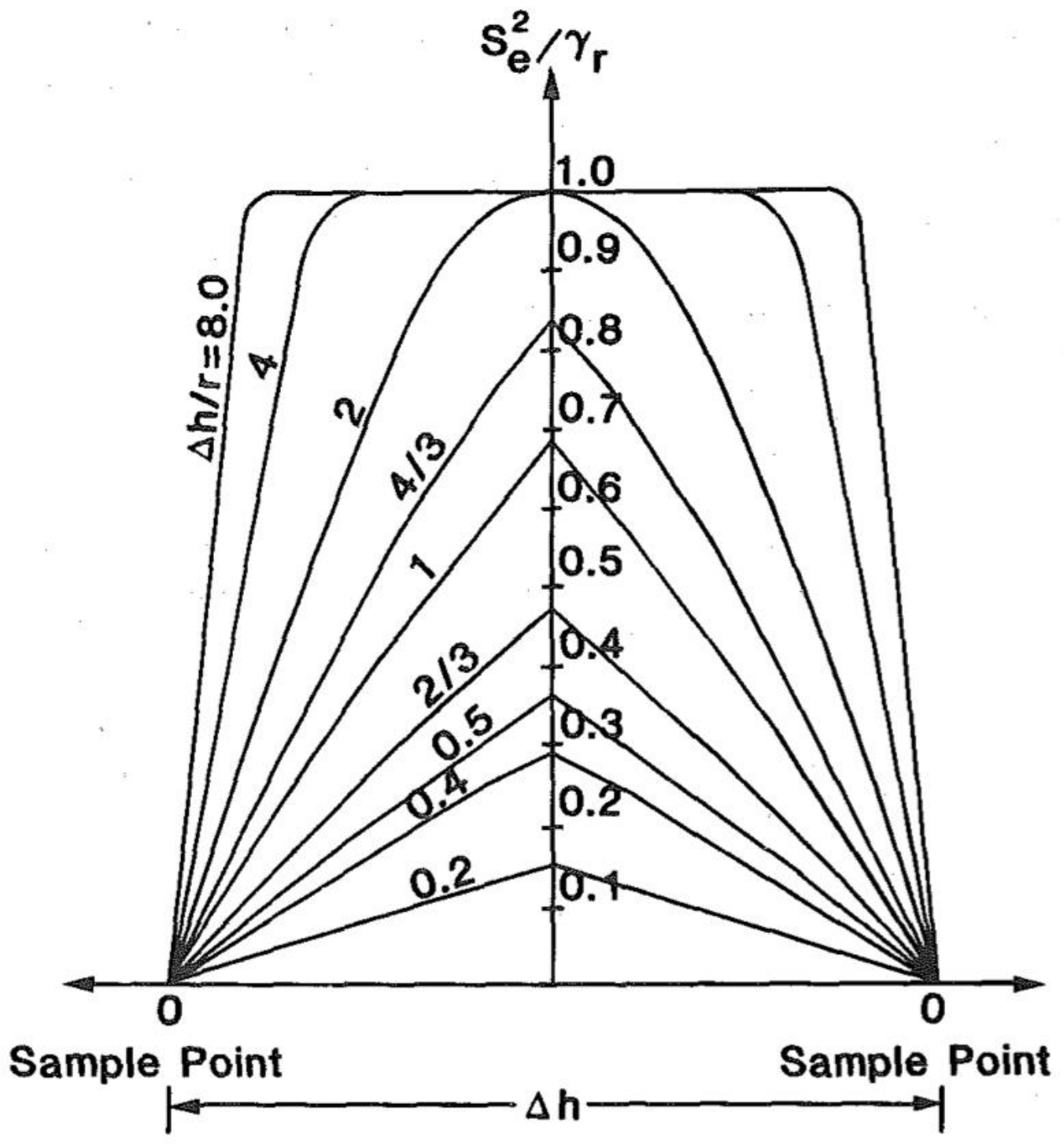

Figure 5.5: Relative error of estimation between sampling points separated by distance (McCuen, 1988)

The figure above can be used to determine the change in accuracy as the spacing in velocity measurement change. 


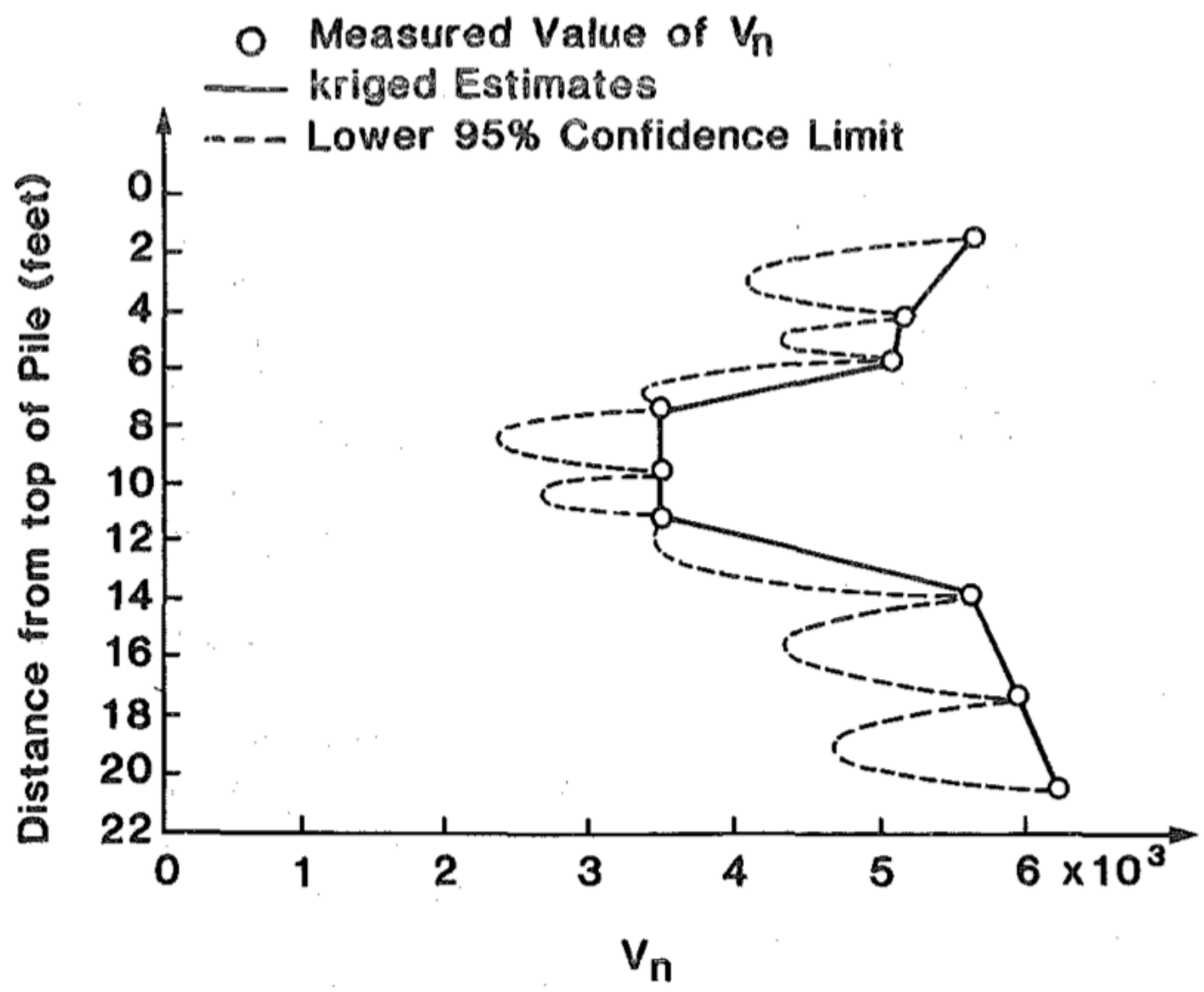

Figure 5.6: Intervals and Kriging estimations (McCuen et al. 1988)

The accuracy improves as the spacing of the points is decreased as shown in the above figure. The above figure also allows for the critical value of the velocity to be located. The critical value provides insight on if the pile needs to be restored or not.

\section{$\underline{\text { Conclusion }}$}

Ultrasonic testing for evaluation of the strength of timber piles happens to be one of the best methods. The tests are more accurate and a cost effective solution compared to other methods. The goal of the case study was to provide insight on the number of measurements to be made in a test and the spacing required. Fewer measurements reduces the time of testing and the cost since the experiment would be shorter. The least amount of measurements while maintaining the highest degree of accuracy would be the ideal scenario. 
The study examined timber piles from four different bridges each consisting of yellowpine timber. The compressive strengths for each piles produced different results and the piles were split between all levels of decay. A spherical semi variogram analyzed the ultrasonic testing measurements in the system. A guideline between the relative accuracy and the relative spacing of point measurements could be calculated using the semi variogram. Confidence intervals were completed to assure the spacing guidelines have a specific level of accuracy even for points in the timber pile where measurements were not taken.

\subsection{CONCLUSION}

Timber structures have been a part of civil infrastructure for many years and will continue to be utilized for years to come. Since the material consists of natural and organic properties, the decay rate is faster than other materials such as concrete and steel. Timber mediums may not be able to reach the compressive strengths levels as some of the other materials, but the properties make it great for smaller projects such as timber bridge piles on a smaller level. Being able to identify any flaws or defects in wooden structures can be critical in determining the safety or usage of a certain structure. Continual advancement in the field will provide these older, wooden structures to be better detected for repair at lower costs. 


\section{CONCLUSIONS AND RECOMMENDATIONS}

\subsection{CONCLUSIONS}

The paper presents an in-depth literature review of Ultrasonic techniques in NDT. Several materials, concrete, steel, and timber are assessed and reviewed by multiple types of tests. The following conclusions can be derived from the literature review:

- The field of NDT techniques and Ultrasonics have taken major leaps in advancement and understanding since first being introduced. The progression for equipment and machinery advanced to smaller and more powerful computing power allowing for faster results in the laboratory or field measurements.

- The chapters in the literature review discussed the applications of ultrasonic techniques for different materials and provided insight on the way cost-effective solutions would advance the understanding leading to future work.

- The report discusses various methods of ultrasonic testing to eliminate the destruction of material, while still producing the most accurate results for cost implications and ease of testing for operators.

- Implementation of ultrasonic techniques still pose limitations since the measurements do not always settle all sought after information. Multiple techniques in NDT can be used to supplement one another since techniques normally have a specialty and do not answer all the required information at the same level of accuracy or speed.

- Limitations of limited experimentation on new materials and areas of emphasis cause the setback of having certainty when testing. Secondary destructive tests still play a role in the evaluation of material properties to ensure the results recorded meet standards. Repeated experiments using the NDT techniques would eliminate or minimize the use of the destructive testing.

- Continued knowledge and updated information would help alleviate the uncertainty utilizing ultrasonic techniques. The more widespread the technique becomes the more affordable, user friendly equipment and software will increase in the field. 
- Future research and new manuals/publications may be useful in educating the general public on the basic theories and ease of application for the field of NDT, and challenge other outdated methods.

\subsection{RECOMMENDATIONS}

- Universities and other leaders in the field of NDT techniques should reach out to larger structural evaluation companies and collaborate to try and implement more practical research into ultrasonic testing.

- State and Federal construction should require use of ultrasonics in testing alongside the already established destructive methods to allow the advancement of ultrasonics.

- Improvement in ultrasonic testing equipment is needed to ensure faster, more accurate work while still providing the easiest accessibility and user-friendly interactions.

- Continual research and information about ultrasonic testing can provide new areas and fields of improvement for structural condition assessment and could lead to new discoveries. 


\section{REFERENCES}

1. Alyousef, R. (2016). The Fatigue Behaviour of Tension Lap Spliced Reinforced Concrete Beams Strengthened with Fibre Reinforced Polymer Wrapping. PhD thesis, University of Waterloo, Civil Engineering.

2. Ashbrook. T (2021). Use of Ultrasonics for Condition Assessment of Concrete. CE 564 Class Paper. West Virginia University, Morgantown, WV.

3. Bennon, Michael and Kim, M. Julie and Levitt, Raymond E., U.S. Infrastructure Gap(s): Federal Policy and Local Public Institutions (September 13, 2017).

4. Blitz, J., Simpson, G. (1996). Ultrasonic Methods of Non-Destructive Testing. Chapman \& Hall, 2-6 Boundary Row. First edition

5. Bompan, K., Haach, V. (2018). Ultrasonic tests in the evaluation of the stress level in concrete prisms based on the acoustoelasticity. Construction and Building Materials. Vol. 162, pg. 740750.

6. Breysse, D. (2010). Deterioration processes in reinforced concrete: an overview. In C. R. Maierhofer, Non-destructive evaluation of reinforced concrete structures (Vol. 1: Deterioration processes and standard test methods, pp. 28-56). Boca Raton, FL: Woodhead Publishing Limited.

7. Bucur, V. (1995/2006). "Acoustics of Wood" In Timell, T. E., and Wimmer, R. (eds.) Acoustics of Wood: $2^{\text {nd }}$ Edition, Springer Series of Wood Science, New York.

8. Bungey, J. H., Millard, S. G., and Grantham, M. G. (2006). "Testing of Concrete in Structures, $4^{\text {th }}$ Edition." Taylor and Fransis, 270 Madison Ave, New York, NY 10016, USA.

9. Chapagain, K. R., Bjerke, W., Melandsø, T., and Wagle, S. (2018). "In situ inspection of concrete structures using a rolling ultrasound scanner." Proceedings of the International Symposium Non-Destructive Testing in Civil Engineering (NDT-CE 2018), New Jersey, USA.

10. Chirp Microsystems. (2020). Ultrasonic Module Pulse-Echo Test Procedure. Chirp Microsystems. AN-000169

11. Ensminger, D., \& Bond, L. (2012). Ultrasonics Fundamentals, Technologies and Applications (Third edition ed.). Boca Raton, Florida, United States: CRC Press. 
12. Feio, A.O.; Lourenço, P.B.; Machado, J.S. (2007). Non-destructive evaluation of the mechanical behavior of chestnut wood in tension and compression parallel to grain. Int. J. Archit. Herit.Vol. 1, pg: 272-292.

13. Franklin, R., and Halabe, U. B. (2014). "Behavioral Study of Rayleigh, Lamb, and Critically Refracted Longitudinal Waves for Crack Detection in Painted and Rusted Steel Structural Components," European International Journal of Science and Technology (EIJST), 3(7), 252270.

14. Genculu, S. (2007). Structural Steel Welding PDH Course S150. PDH Center

15. Gu, X.P., Xu, G.C., Liu, J., Gu, X.Y.(2013). Ultrasonic Testing and Evaluation of Laser Welds in Stainless Steel. Old Cityy Publishing, Inc. Vol. 26, pp: 103-113.

16. Güçlüer,K. (2020). Investigation of the effects of aggregate textural properties on compressive strength (CS) and ultrasonic pulse velocity (UPV) of concrete. Journal of Building Engineering. Vol. 27.

17. Halabe, U. B., and Franklin, R. (2013). "Detection of Cracks in Long Painted or Rusted Steel Beams Using Ultrasonic Rayleigh Waves,” European International Journal of Science and Technology (EIJST), 2(7), 161-182.

18. Halabe, U. B., and Franklin, R. (2001). "Fatigue Crack Detection in Metallic Members Using Ultrasonic Rayleigh Waves with Time and Frequency Analysis," Materials Evaluation, 59(3), 424-431.

19. Hao, S. (2010). I-35W Bridge Collapse. Journal of Bridge Engineering. Vol. 15(5), pp.608614.

20. He, J., Chen, Z., Lou, X. (2020). A Bayesian Estimation-based Uncertainty Qualifiation of Flaws in Steel Welds Detected by Ultrasound Phased Array. J. Phys.: Conf. Vol. 1592.

21. Juncai Xu, Hai Wei, (2019). Ultrasonic Testing Analysis of Concrete Structure Based on S Transform, Shock and Vibration, vol. 2019, Article ID 2693141, 9 pages.

22. Kaczmarek, R., \& Słania, J. (2018). Interpretation of indications generated by small welding discontinuities in ultrasonic time of flight diffraction technique. Journal of Nondestructive Evaluation, 37(3).

23. Kishore, K. (2014). Ultrasonic Testing of Concrete. Civil Engineering Portal.

24. Knab, L., Blessing, G., Clifton, J. (1983). Laboratory Evaluation of Ultrasonics for Crack Detection in Concrete. Journal Proceedings, Vol. 80, pp:17-27. 
25. Krautkrämer, J. \& Krautkrämer, H. (1990). Ultrasonics Testing of Materials. Springer-Verlag Berlin Heidelberg $\mathrm{GmbH}, 4^{\text {th }}$ Edition.

26. Lipa, L., Navarro, L., Pasquel, E. (2019). Influence of Steel Bars in Detection of Voids in Concrete Using Ultrasonic Pulse-Echo Method. ACI Materials Journal. Title No. 116-M65

27. Lerch, Terence Peter, (1996) "Ultrasonic transducer characterization and transducer beam modeling for applications in nondestructive evaluation ". Retrospective Theses and Dissertations. 11157.

28. Maes, G. (2018) Webinar: Advanced Focusing Techniques for Better UT Inspections [Webinar].

29. McCuen, R. H., Aggour, M. S., \& Ayyub, B. M. (1988). Spacing for accuracy in ultrasonic testing of bridge timber piles. Journal of Structural Engineering, 114(12), 2652-2668.

30. Michaels, J.E., Michaels, T.E. \& Mi, B. (2006). An Ultrasonic Angle Beam Method for in situ Sizing of Fastener Hole Cracks. J Nondestructr Eval 25, 2-15.

31. Nowak T, Karolak A, Sobótka M, Wyjadłowski M. (2019) Assessment of the Condition of Wharf Timber Sheet Wall Material by Means of Selected Non-Destructive Methods. Materials. 12(9):1532.

32. Omar, T., Nehdi, M, L. (2018). "Condition Assessment of Reinforced Concrete Bridges: Current Practice and Research Challenges". Infrastructures, Vol. 3.3.

33. Omar, T., Nehdi, M. L., and Zayed, T. (2018). "Infrared Thermography Model for Automated Detection of Delamination in RC Bridge Decks." Construction and Building Materials, Vol. 168, pp: 313-327.

34. Roblero, M. (2017). Condition Assessment of Concrete Elements Through Two Nondestructive Ultrasonic Techniques. University of Waterloo, Civil Engineering.

35. Rucka M. (2018). Failure Monitoring and Condition Assessment of Steel-Concrete Adhesive Connection Using Ultrasonic Waves. Applied Sciences. 8(3):320.

36. Ryuzono K, Yashiro S, Nagai H, Toyama N. (2020). Topology Optimization-Based Damage Identification Using Visualized Ultrasonic Wave Propagation. Materials. ; Vol. 13(1): Pg: 33

37. Sabbag, Nevbahar \& Uyanik, Osman (2017). Prediction of Reinforced Concrete Strength by Ultrasonic Velocities. Journal of Applied Geophysics, Vol. 141, pp: 13-23. 
38. Shokouhi P, Riviere J, Lake C, Bas P, Ulrich T.J. (2017). Dynamic Acousto-elastic Testing of Concrete with a Coda-wave Probe: Comparison with Standard Linear and Nonlinear Ultrasonic Techinque. Ultrasonics. Vol.81, pg: 59-65.

39. Słoński, M., Schabowicz, K., Krawczyk, E. (2020). Detection of Flaws in Concrete Using Ultrasonic Tomography and Convolutional Neural Networks. Materials 13, no. 7: 1557

40. Song, C., Kim, YJ., Cho, CB., Chin, WJ., Park, K-Y. (2020). Estimation Length of Anchor Bolt Inside Concrete Using Equation for Arrival Time and Shortest Time Path of Ultrasonic Pulse. Appl. Sci. 10, 8848.

41. Stephens, R.W.B. (1975). An Historical review of ultrasonics, Proceedings, Ultrasonics International. pg. 9-19, ICP Press, Guildord, UK, 1975

42. Teng KH, Kot P, Muradov M, Shaw A, Hashim K, Gkantou M, Al-Shamma'a A. (2019). Embedded Smart Antenna for Non-Destructive Testing and Evaluation (NDT\&E) of Moisture Content and Deterioration in Concrete. Sensors. 19(3):547.

43. Tian, Fangyuan, Yanpeng Hao, Zhouyiao Zou, Yao Zheng, Weiming He, Lin Yang, and Licheng Li. 2019. "An Ultrasonic Pulse-Echo Method to Detect Internal Defects in Epoxy Composite Insulation" Energies 12, no. 24: 4804. https://doi.org/10.3390/en12244804

44. Velay-Lizancos, M., Martinez-Lage, I., Azenha, M., Granja, J., Vazquez-Burgo, P. (2018). Concrete with Fine and Coarse Recycled Aggregates: E-modulus Evolution, Compressive Strength and Non-Destructive Testing. Construction and Building Materials. Vol. 193, pg: 323-331.

45. Washer, G., Fuchs, P., Graybeal, B. A., \& Hartmann, J. L. (2004). Ultrasonic testing of reactive powder concrete. Ieee Transactions on Ultrasonics, Ferroelectrics, and Frequency Control, 51(2). https://doi.org/10.1109/TUFFC.2004.1320767

46. Wolf, J., Milmann, B., Helmerich, R., Kopp, C., Mielentz, F., Wiggenhauser, H., Kurz, J. H., Moryson, R. M., Samokrutov, A., Alekhin, S., Alver, N., and Sazak, H. O. (2014). "Embedded Ultrasound Sensors for Monitoring Concrete Structures.” Proceedings of Structural Materials Technology for Highways and Bridges (SMT 2014).

47. Wroblewski, R., Stawiski, B. Attempt to estimate fire damage to concrete building structure. Arch. Civ. Mech. Eng. 2006, 6, 23-29 
48. Wronkowics, A., Dragan, K., Lis, K. (2018). Assessment of Uncertainty in Damage Evaluation by Ultrasonic Testing of Composite Structures. Composite Structures. Vol. 203. Pg: 71-84.

49. Yuan, W., Guo, A., \& Li, H. (2017). Seismic failure mode of coastal bridge piers considering the effects of corrosion-induced damage. Soil Dynamics and Earthquake Engineering. Vol 93. pp. 135-146.

50. Zamen, S., \& Dehghan-Niri, E. (2020). Fractural Analysis of Nonlinear Ultrasonic Waves in Phase-Space Domain as a Quantitative Method for Damage Assessment of Concrete Structures. Elsevier Ltd

51. Zielińska, M., \& Rucka, M. (2020). Detection of Debonding in Reinforced Concrete Beams Using Ultrasonic Transmission Tomography and Hybrid Ray Tracing Technique.Construction Building Material.

52. Zhitlukhina, Y., Perov, D. (2008) A Possibility of Using Transverse Ultrasonic Waves to Obtain Information on Microflaws in Steel. Journal of Nondestructive Testing. Vol. 44, No.6, pp. 62-75. 